\title{
A Survey of Optical Carrier Generation Techniques for Terabit Capacity Elastic Optical Networks
}

\author{
Muhammad Imran, Prince M. Anandarajah, Senior Member, IEEE, Aleksandra Kaszubowska-Anandarajah, \\ Member, IEEE, Nicola Sambo and Luca Poti, Senior Member, IEEE
}

\begin{abstract}
Elastic optical networks (EON) have been proposed to meet the network capacity and dynamicity challenges. Hardware and software resource optimization and re-configurability are key enablers for EONs. Recently, innovative multi-carrier transmission techniques have been extensively investigated to realize high capacity $(\mathrm{Tb} / \mathrm{s})$ flexible transceivers. In addition to standard telecommunication lasers, optical carrier generators based on optical frequency combs (OFC) have also been considered with expectations of reduced cost and inventory, improved spectral efficiency and flexibility. A wide range of $\mathrm{OFC}$ generation techniques have been proposed in the literature over the past few years. It is imperative to summarize the state of the art, compare and assess these diverse techniques from a practical perspective. In this survey, we identify salient features of optical multicarrier generators, review and compare these techniques both from a physical and network layer perspective. OFC demultiplexing/filtering techniques have also been reviewed. In addition to transmission performance, the impact of such sources on the network performance and real-world deployment strategies with reference to cost, power consumption, and level of flexibility have also been discussed. Field trials, integrated solutions, flexibility demonstrations are also reported. Finally, open issues and possible future directions that can lead to real network deployment are highlighted.
\end{abstract}

Index Terms-Optical frequency combs, Multi-wavelength sources, Sliceable Bandwidth Variable Transponders (SBVT), Elastic optical networks.

\section{INTRODUCTION}

$\mathbf{O}$ PTICAL frequency combs (OFCs) and their implementation were well documented by early reports in the 60's and 70's [1] including the Nobel lecture by Hansch [2] and Hall [3]. In the last decade or so, there has been an immense amount of research activity focusing on OFCs and their wide range of applications including molecular spectroscopy[4], astronomy [5], RF photonics [6], optical clocks [7], arbitrary waveform generation [8], and optical communications [9]. An OFC can be defined, as a series of equally spaced discrete spectral lines [10]. There are various parameters that could be used to characterise an OFC and the choice of ideal ones depends on the nature of the application. The requirements for comb features like frequency and amplitude stability, occupied bandwidth, free spectral range (repetition rate), spectral

M. Imran and N. Sambo are with the TeCIP institute, Scuola Superiore Sant'Anna, Pisa, 56127 Italy e-mail: (m.imran@ santannapisa.it).

Prince M. Anandarajah is with the School of Electrical Engineering, Dublin City University, Ireland.

Aleksandra Kaszubowska-Anandarajah is with the Connect Centre, Trinity College Dublin, Ireland.

Luca Potì is with the CNIT, Pisa, Italy.

Manuscript received March 30, 2017 flatness, phase noise, phase correlation etc. differ depending on the application. An impressive report on the evolution of mode-locked femtosecond laser based combs and their various applications can be found in [11] and [12], while an excellent tutorial on OFC technologies for ultra-broadband radio photonics has been reported by Torres et al. [10]. Different to previous surveys, in this paper we focus on recent advances in OFCs for employment in flex-grid/elastic optical networks. While OFCs have been envisioned for WDM/TDM fixed-grid optical communications for many years as an alternative to tens of individual lasers or arrays of lasers, they have not yet been commercially deployed [13].

Emerging broadband applications and bandwidth hungry dynamic services are driving the evolution of optical networks. These new services generate dynamic traffic and demand a high quality of service (QoS). As a result, next generation optical networks would not only need to provide an increased capacity and reach, but also be required to respond to dynamic traffic and network conditions. Conventional fixed grid WDM networks provide fix rate $10 \mathrm{~Gb} / \mathrm{s}, 40 \mathrm{~Gb} / \mathrm{s}$ and recently 100 $\mathrm{Gb} / \mathrm{s}$ connections between the nodes with the limited flexibility enabled by tunable lasers and reconfigurable switches. Recently, $400 \mathrm{~Gb} / \mathrm{s}$ and $1 \mathrm{~Tb} / \mathrm{s}$ transponders, employing high order spectrally efficient modulation techniques, have also been proposed. However, in such a multi-rate connection scenario, the modification or upgrade of the network, to meet the dynamic requirements, is an extremely challenging task. Moreover, addressing this issue will prove to be expensive, mainly due to the inventory cost.

The concept of flexible /elastic optical networking has been proposed to realize a network that is able to dynamically adjust its resources based on traffic and network conditions [14], [15]. The flexibility has been envisioned across the network layers, involving flexible spectral grid management, transmission, switching, planning and operation as well as control plane functions. Flexible transceivers, also referred as sliceable bandwidth variable transponders (SBVT) or multiflow transponders, are expected to tune their parameters (modulation format, baud rate, number of subcarriers etc.) to serve varying capacity and different reach requirements. Advanced multicarrier transmission techniques, like Nyquist wavelength division multiplexing (NWDM) [16], coherent optical orthogonal frequency division multiplexing (CO-OFDM) [17], [18], time frequency packing (TFP) [19], have been investigated to realize terabit flexible transponders. By densely packing several channels, called subchannels/subcarriers, spectrally efficient superchannels are formed, thus providing increased 


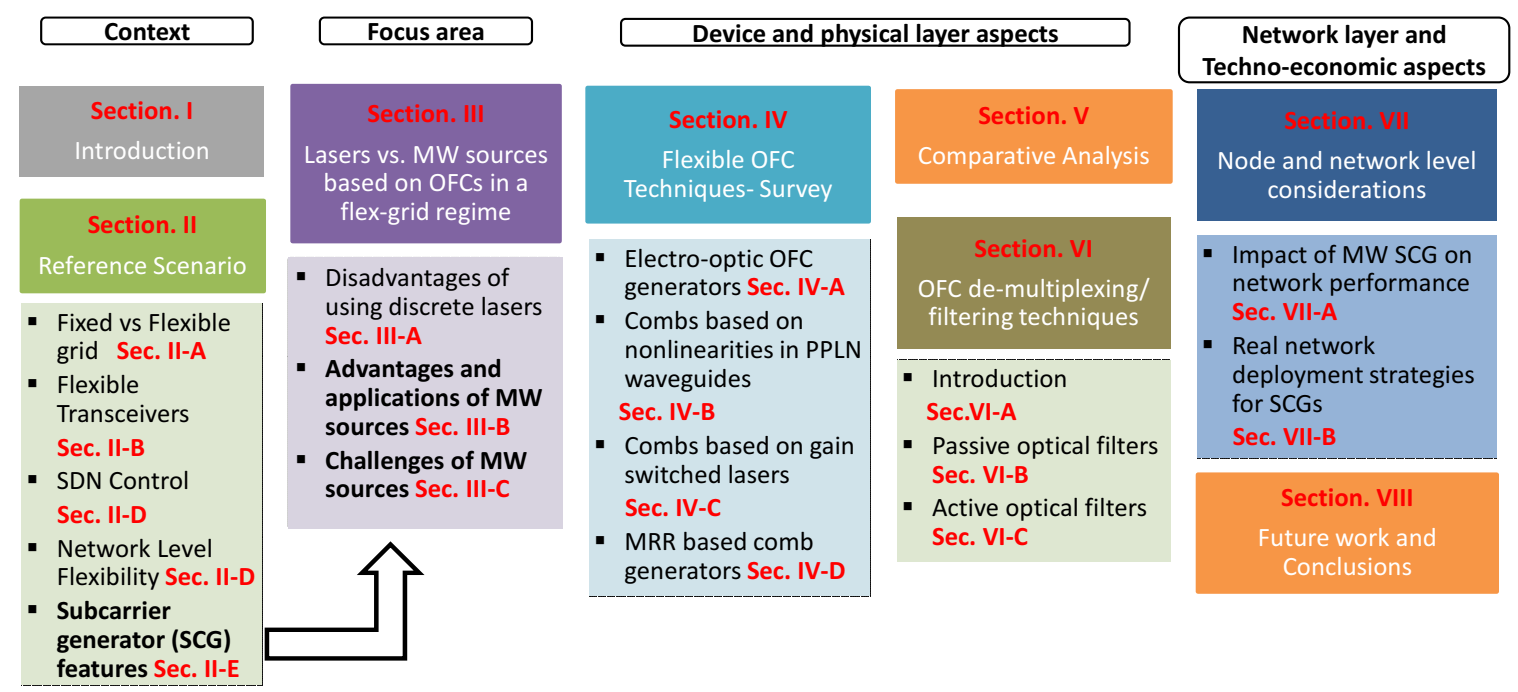

Figure 1: Survey Organization

network capacity.

The optical carriers in SBVTs can be generated by a conventional array of independent lasers (standard integrated tunable laser assembly (ITLAs) or an integrated array). However, such a multi-laser SBVT (ML-SBVT) solution suffers from frequency drift (variable frequency spacing) and lacks any phase commonality (or phase correlation) between the carriers. An alternative would be to use an OFC-based multi-wavelength SBVT (MW-SBVT), in which a single semiconductor device provides multiple optical frequencies that are phase coherent, equally spaced and can be easily and simultaneously tuned in wavelength. In the past, OFCs were proposed to be used in WDM optical communication due to their expected cost and power efficiency (replacing multiple lasers with single device). Recently, the need for flexibility and tight channel spacing, has lead to a renewed interest in OFCs. The search for an optimum solution for elastic optical networks, has resulted in several OFC generation techniques being proposed. These methods are described in section IV of this paper. Note: although semiconductor and fiber based mode locked lasers (MLLs) are a very popular type of OFC generators, they are beyond the scope of this study, which focuses on OFCs for Terabit capacity elastic optical networks, where flexibility in terms of wavelength and free spectral range (FSR) tuning is critical.

Survey Contributions and Organization: In this paper we review, compare, and contrast different OFC generation techniques from the perspective of their applicability in elastic optical networks. The aim of the analysis is to provided answers to the following questions: "Can current OFCs really replace an array of lasers (or conventional lasers)?"and "what are the obstacles to their use in a commercial systems?". To the best of our knowledge, it is the first time the question of OFC employment in the elastic optical networks has been explored from a practical perspective. The contributions of this work include:

i) Comparative analysis of conventional lasers and OFC-based sources in the EON. ii) Survey of flexible OFC generation and demultiplexing/filtering techniques.

iii) Comparative analysis and trade-offs of candidate OFC technologies.

iv) Identification of key considerations for node deployment of the MW-SBVT and its impact on network performance.

The survey organization is pictorially represented in the Fig. 1. In Section II a typical application scenario is presented, briefly describing the elastic optical networking paradigm, with reference to an envisioned transceiver, SDN control and network flexibility. The desired characteristics of an optical carrier generator subsystem are outlined and its relationship to both network and transceiver flexibility is discussed. The issues of standard telecommunication lasers and advantages/applications of OFC based multiwavelength (MW) sources in the flex-grid system are presented in Section III. Several key challenges of MW sources are also discussed. Section IV provides the detailed survey of recently proposed flexible OFC generation techniques for elastic optical networking. A comparative analysis of the reviewed techniques is presented in Section V. A summary of recent comb based flexible transceiver demonstrations, field trials and integrated efforts, showing the feasibility of using such sources, is also provided. OFC de-multiplexing/filtering techniques are reviewed in Section VI. Essential node and network level considerations, with reference to the network performance and the node architecture, are detailed in Section VII. Different optical carrier source deployment strategies are also discussed. Finally, based on the comparative study, we highlight several open issues that still need to be addressed, in order to make comb based MW sources a technically and financially viable alternative to an array of semiconductor lasers.

\section{LIST OF ACRONYMS}

ADC Analog to Digital Converter

AFC Automatic Frequency Control 


\begin{tabular}{|c|c|c|c|}
\hline AMZI & Asymmetric Mach Zehnder Interferometer & NOLM & Nonlinear Optical Loop Mirror \\
\hline API & Application Program Interface & NWDM & Nyquist Wavelength Division Multiplexing \\
\hline AWG & Arrayed Waveguide Gratings & OAM & Operations Administration and Management \\
\hline BAPE & Brillouin-Assisted Power Equalizer & OCSM & Optical Carrier Supply Module \\
\hline BPF & Band-Pass Filter & OEO & Optoelectronic Oscillators \\
\hline CAPEX & Capital Expenditure & OFC & Optical Frequency Combs \\
\hline CBW & Comb Bandwidth & OPEX & Operational Expenditures \\
\hline CFBG & Chirped Fiber Bragg Gratings & OSNR & Optical Signal to Noise Ratio \\
\hline CFS & Complementary Frequency Shifter & OTDM & Optical Time Division Multiplexing \\
\hline OFDM & Orthogonal Frequency Division Multiplexing & PCB & Printed Circuit Board \\
\hline CSiB & Carrier Source in Board & PIC & Photonic Integrated Circuit \\
\hline CSiN & Carrier Source in Node & PLC & Planar Lightwave Circuits \\
\hline CSiNet & Carrier Source in Network & PM & Phase Modulator \\
\hline $\mathbf{C W}$ & Continuous Wave & PolM & Polarization Modulator \\
\hline DAC & Digital to Analog Converter & PPLN & Periodically Poled Lithium Niobate \\
\hline DBR & Distributed Bragg Reflection & QAM & Quadrature Amplitude Modulation \\
\hline DCF & Dispersion Compensating Fibre & QoS & Quality of Service \\
\hline DFG & Difference Frequency Generation & QPM & Quasi-Phase Matching \\
\hline DM & Discrete Mode & QPSK & Quadrature Phase Shift Keying \\
\hline DPMZM & Dual Parallel MZM & RFS & Re-circulating Frequency Shifting \\
\hline DSF & Dispersion Shifted Fibre & $\mathbf{R I N}$ & Relative Intensity Noise \\
\hline DSP & Digital Signal Processing & ROADM & Reconfigurable Optical Add Drop Multiplexer \\
\hline EO & Electro-Optic & RRC & Root Raised Cosine \\
\hline EON & Elastic Optical Networks & $\mathbf{R S A}$ & Routing and Spectrum Assignment \\
\hline $\mathbf{E R}$ & Extinction Ratio & RSTA & Routing, Spectrum and Transponder Assignment \\
\hline FEC & Forward Error Correction & SBS & Stimulated Brillouin Scattering \\
\hline FoM & Figure of Merit & SBVT & Sliceable Bandwidth Variable Transponders \\
\hline FSC & Flex Subcarrier & SCG & Subcarrier Generation \\
\hline FSR & Free Spectral Range & SDN & Software Defined Network \\
\hline FWM & Four Wave Mixing & SE & Spectral Efficiency \\
\hline GSL & Gain Switched Laser & SFG & Sum Frequency Generation \\
\hline GVD & Group Velocity Dispersion & SHG & Second Harmonic Generation \\
\hline HNLF & Highly Nonlinear Fiber & SMSR & Side Mode Suppression Ratio \\
\hline ICI & Inter Carrier Interference & SOH & Silicon Organic Hybrid \\
\hline IM & Intensity Modulator & SOI & Silicon-On Insulator \\
\hline ISI & Inter Symbol Interference & SPM & Self-Phase Modulation \\
\hline ITLA & Integrated Tunable Laser Assembly & SSB-SC & Single Side Band - Suppressed Carrier \\
\hline ITU & International Telecommunication Union & SSMF & Standard Single Mode Fibre \\
\hline LCOS & Liquid Crystal On Silicon & TFP & Time Frequency Packing \\
\hline LDPC & Low Density Parity Check & TLS & Tunable Laser Source \\
\hline LO & Local Oscillator & TNR & Tone-to-Noise Ratio \\
\hline MLL & Mode Locked Lasers & WDM & Wavelength Division Multiplexing \\
\hline MRR & Micro Ring Resonators & WL & Wavelength Locker \\
\hline MW & Multi-Wavelength & WSS & Wavelength Selective Switch \\
\hline
\end{tabular}




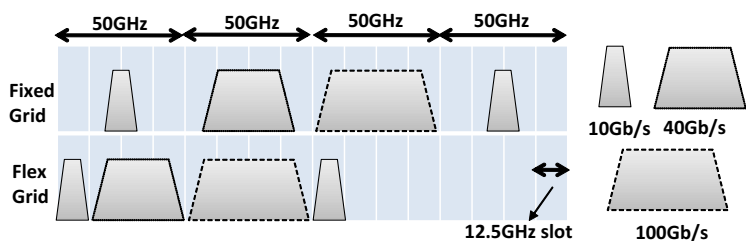

Figure 2: Fixed grid vs Flex-grid.

\section{REFERENCE SCENARIO}

Optical metro and core transport networks carry diverse traffic and are designed to be transparent (protocol independent), backward compatible and future proof. In conventional fixed WDM networks, the bandwidth-distance product has been employed as a key performance indicator. Unpredictability of dynamic traffic is an additional challenge to network performance. It is hard to predict when and where new traffic requirements may arise, resulting in a higher degree of uncertainty. Efficient operations, administration and management (OAM), high QoS and lower capital and operational expenditures (CAPEX and OPEX) are key network performance challenges faced by networks' operators. These need to be overcome in order to ensure customer satisfaction and profitability. Efficient resource utilization and re-configurability (flexibility) in hardware and software can help to meet these challenges. In addition, flexible hardware, such as transceivers and switches (e.g. bandwidth variable cross-connects or spectrum selective switches) as well as flexible control plane, are also considered essential tools. A flexible optical networking paradigm has been proposed to provide all these features and the interested reader interested can refer to [15], [20], [21] for more details. In this manuscript, we focus on flexible transceivers and the network operation aspects directly dependent/related to them.

\section{A. Fixed vs Flexible grid}

The International Telecommunication Union Telecommunication Standardization Sector (ITU-T) fixed $50 \mathrm{GHz}$ grid wavelength allocation plan accommodates 80 channels, by dividing the $4 \mathrm{THz}$ C-band spectrum into $50 \mathrm{GHz}$ slots. Each channel is assigned a defined central frequency $\mathrm{f}$ and $50 \mathrm{GHz}$ of spectrum $(f-25 \mathrm{GHz}$ to $f+25 \mathrm{GHz})$ around the central frequency. Currently deployed 10,40 , and $100 \mathrm{~Gb} / \mathrm{s}$ transponders can fit into these slots, as shown in Fig. 2. A move towards higher data rates, such as $400 \mathrm{~Gb} / \mathrm{s}$ and 1 $\mathrm{Tb} / \mathrm{s}$, would require larger spectral bandwidth, which can be created by joining multiple $50 \mathrm{GHz}$ slots. However, since the optical switches conforming to fixed grid, would not be able to switch these broader channels, a new type of flexible and reconfigurable optical switch would have to be employed in the future networks. [15].

For optimum utilization of spectral resources and to accommodate heterogeneous bandwidth demands, the ITU-T has extended its recommendations G.694.1 [22] and G.872 [23], by including a flexible grid concept. The flex-grid recommendation allows for a flexible division of the optical spectrum and defines the concept of a frequency slot, in addition to a nominal central frequency and a channel/carrier spacing. A frequency slot is defined by its nominal central frequency and its slot width. The first is defined as $193.1+n \times 0.00625 \mathrm{THz}$, where $n$ is a positive or negative integer. The slot width is defined as $12.5 \mathrm{GHz} \times m$, where $m$ is a positive integer. Thus the minimum slot width is $12.5 \mathrm{GHz}$. A channel's nominal central frequency identifies its position in the spectrum and slot width determines the occupied spectrum. Different slot widths can be allocated to accommodate different spectral needs.

\section{B. Flexible Transceivers}

The effective capacity, transparent reach, spectral efficiency, and tunability are considered lightpath connection level performance metrics for flexible transceivers. The spectral efficiency (SE), measured in $(\mathrm{b} / \mathrm{s} / \mathrm{Hz})$, is the ratio of effective capacity (b/s without overhead) over the required transmission spectrum (Hz). Tunability refers to the ability to adapt capacity, transparent reach and SE based on connection needs and transceivers that can provide this flexibility are referred to as bandwidth variable transceivers (BVT) [20]. Fig. 3 shows the various transmission parameters that can be simultaneously adjusted, in order to achieve the desired SE, capacity and reach. For a single carrier transmitter, data rate can be increased either by increasing the baud rate (symbol rate) or by using a higher order modulation format. Furthermore, the information rate can be doubled by the use of polarization multiplexing, which allows for transmission of independent data stream on each polarization state. While the above-mentioned solutions increase the SE and effective capacity of the network, they do so at the expense of the lower transmission reach. The latter can in turn be improved, by increasing the strength of the error correction code (increasing the number of redundant bits), but at an expense of reduced information rate. Another route to increased SE is the application of a multichannel transmission. This solution uses a group of closely spaced multiple optical carriers, referred to as a superchannel, that travel across the network in parallel. A superchannel is considered as a single manageable entity and the constituent channels are termed as optical subcarriers. By combining several lower speed subcarriers, the superchannel achieves a $\mathrm{Tb} / \mathrm{s}$ transmission, while using state-of-the-art analog-to-digital converters and avoiding the challenges and penalties arising from increasing the line rate. Electrical carrier multiplicity can be used to control the bit rate and occupied bandwidth on a finer scale. NWDM, CO-OFDM and TFP are some of the enabling multicarrier technologies that can be used to achieve a terabit transmission.

In BVTs full capacity of the transmitter is allocated to single source-destination pair, which results in a underutilization of resources. To ensure higher utilization and financial viability, multi-flow transponders or SBVTs, have been proposed. An SBVT is capable of generating multiple optical flows that can be routed to different destinations, thus sharing the capacity of a single transponder between multiple source-destination pairs [14], [15], [24]. 


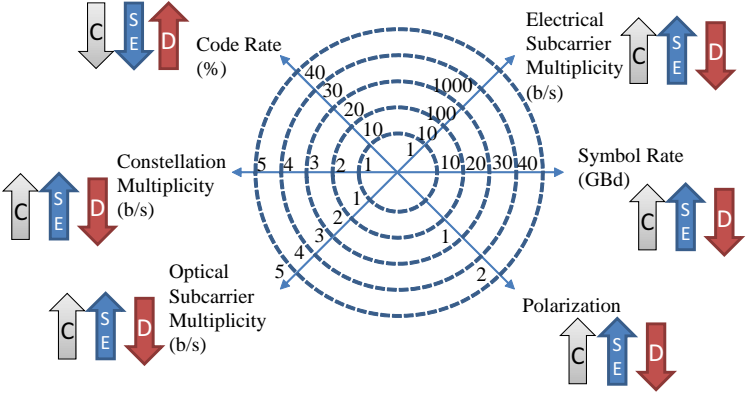

Figure 3: Transceiver flexibility wheel. C - capacity, D distance, SE - spectral efficiency.

1) SBVT architecture: A SBVT can be considered as a collection of "virtual" lower capacity BVTs, logically assembled in groups, to generate independent superchannels. The reference SBVT architecture for our study is shown in Fig. 4 [24]. The client signals (OTN or Ethernet) are adapted for the photonic layer by a sliceable OTN interface (not shown) that creates different data streams, by fragmenting incoming traffic. The streams are fed to flow distributers of different multiflow optical modules (i.e. SBVTs). The flow distributor is a reconfigurable electronic switch matrix and it directs different OTN streams to specific flex subcarrier (FSC) modules for carrier modulation. The subcarrier generation (SCG) module is responsible for the provision of optical carriers for modulation in the transmitter and for local oscillators for coherent detection in the receiver. The SCG options are shown in Fig. 4 (bottom) and include:

i) an array of individual ITLA lasers

ii) an integrated laser array

iii) a flexible MW source based on OFCs [25].

This SCG module is the main focus of this survey. A SCG module can be connected to a FSC module via a printed circuit board (PCB), a planar lightwave circuits (PLC) or an optical back plane. Since the idea of a superchannel relies on optical parallelism, the transmitter and receiver resources have to be replicated. Therefore for practical reasons, the number of subcarriers in an SBVT should be kept reasonably low e.g., less than ten. The FSC is a flexible transceiver responsible for transmitting and receiving data flows. The transmitter part in a FSC has a digital signal processing (DSP) block, a digital to analog converter (DAC) and an IQ modulator. The receiver part consists of a coherent receiver, an analog to digital converter (ADC) and a receiver DSP block. The DSP in the transmitter performs functions such as encoding, pulse shaping and pre-distortion, while the DSP in the receiver is used for impairment mitigation, clock recovery, synchronization etc. [26]. The coherent detector and the IQ modulator are expected to be pluggable modules. Due to the power constraints of pluggable modules, lasers are not included in a transceiver case and are proposed to be integrated on the line card [27]. Optical subchannels from different FSCs can be coupled/ multiplexed to form a superchannel and enter the network through add ports of flex OXC of node.

2) Transmission techniques for SBVT: Different implementations of FSM have been investigated, with the main three
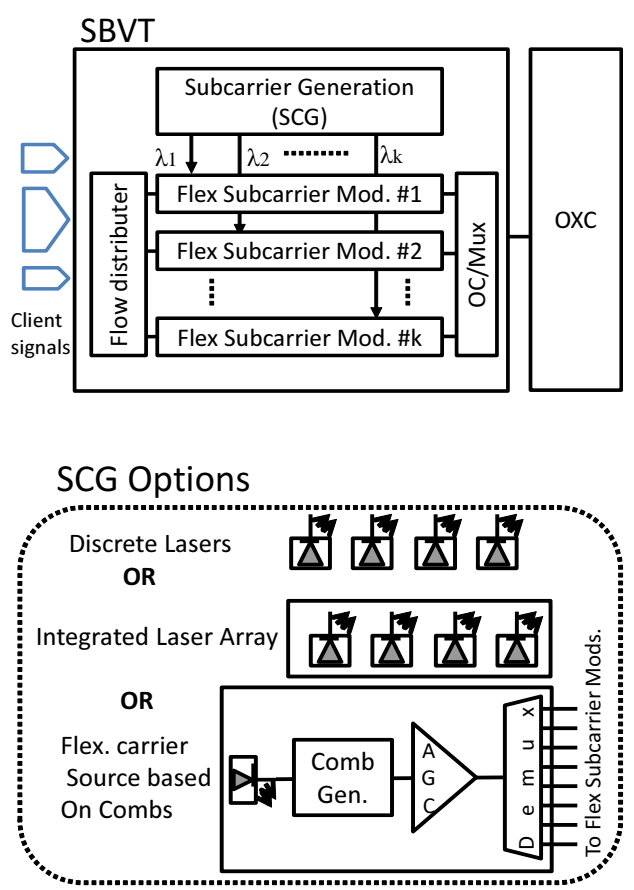

Figure 4: SBVT architecture and SCG options.

being NWDM, CO-OFDM and TFP. These techniques differ in the achieved SE, reach, cost and complexity. The choice of the optimum technique depends on the application scenario, network architecture and financial affordability. A comparative analysis of the techniques can be found in [16], [24], [28], [29]. The quest for a high SE requires the channel spacing to be minimized, which may cause crosstalk or inter carrier interference (ICI). One solution to this problem may be prefiltering of channels at the transmitter (Tx), in order to avoid ICI (frequency domain). However, this in turn may cause inter symbol interference (ISI) (time domain). For optimum transmission performance, both ICI and ISI must be eliminated or minimized. The ratio of channel spacing $(\Delta f)$ and symbol rate (B) can be used to classify different WDM schemes from the SE perspective. NWDM and OFDM technique have $(\Delta f) / \mathrm{B}<1.2$, whereas TFP has $(\Delta f) / \mathrm{B}<1$, thus is referred to as faster than Nyquist [30].

NWDM: In principle, the NWDM technique consists of transmitting channels having an ideal rectangular spectral shape with the bandwidth equal to the symbol rate and a sinc like temporal profile. The main idea is to apply the pulse shape filters at the transmitter to confine the channel bandwidth within the Nyquist frequency of the signal (for ISI free transmission), thus enabling dense spectral allocation of sub-channels. In practical implementations, root raised cosine (RRC) filters are used for pulse shaping and matched filters are used at the receivers, to reduce the ISI due to narrow filtering [24]. A certain number of NWDM sub-channels are then multiplexed, with a hugely reduced guard band, to realize a superchannel. Thus NWDM first attempts to minimize the spectral occupancy of each subchannel and then closely pack them to reduce the spectral guard bands, thereby increasing the overall SE. A typical spectrum of NWDM signals is shown in 
(a)

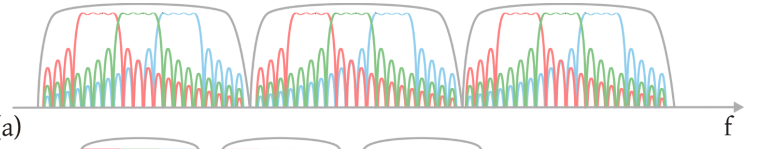

(b)

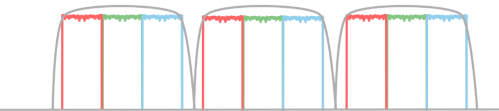

(c)

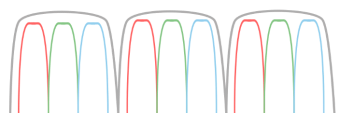

(d)

Figure 5: Typical spectrum for different transmission techniques (a) OFDM with 4 subcarriers, (b) OFDM with 256 subcarriers, (c) NWDM and (d) TFP [24].

Fig. 5 (c).

CO-OFDM: A coherent optical OFDM signal consists of multiple, mutually orthogonal subcarriers that overlap in the frequency domain, providing higher SE. While the NWDM signals have a rectangular spectral profile, with a bandwidth equal to the symbol rate and sinc like pulse shape in the time domain, the CO-OFDM transmits ideal rectangular pulses of duration equal to a symbol time and a sinc-like spectrum. The crosstalk, due to overlapping of the subcarriers, is canceled using proper filtering in the receiver.

The CO-OFDM signal can be generated either in the electrical or all-optical manner. In the first case, the subcarriers are created in the digital domain, using the Inverse Fast Fourier Transform (IFFT) and used to modulate an optical carrier. The IFFT-based OFDM generation provides great flexibility in terms of the number of subcarriers and their separation, thus achieving fine granularity, bit rate scalability and easy spectrum shaping [31]. The disadvantage of this method is its reliability on digital-to-analog converters (DACs), whose bandwidth limits the maximum achievable bandwidth of the OFDM signal. All-optical OFDM on the other hand, entails modulating data signals directly onto optical subcarriers. The optical subcarriers should be frequency locked, having carrier spacing equal to symbol rate, and time aligned symbols on all subcarriers (i.e. symbols having synchronous transitions) [32], [33]. The main advantage of the optical approach is that the electronics of the DACs are eliminated, thus removing the constraints these devices place on the achievable transmission rates. However, in order to limit the cost and complexity of the systems, the number of subcarriers is usually much lower than in the IFFT-based systems. As increasing the number of subcarriers helps to achieve a better (i.e. more rectangular) spectrum shape, all-optical OFDM suffers from large out-ofband emission and require greater separation between the adjacent superchannels, in order to avoid interchannel interference. The impact of the number of the OFDM subcarriers on the signal spectrum is demonstrated in Fig. 5 (a) and (b). Both figures show spectra of 3 superchannels, each consisting of 3 optical subchannels (red, green and blue), but with different number of OFDM subcarriers: in Fig. 5 (a) a subchannel carries 4 OFDM subcarriers (visible as small bumps in the spectrum), while in Fig. 5 (b), each subchannel carriers 256 subcarriers.

TFP: By exploiting the time frequency packing technique (TFP), each individual subcarrier's bandwidth and separation between the subcarriers can be significantly reduced to achieve a much higher SE. It is faster than the Nyquist technique, for which $(\Delta f) / \mathrm{B}<1$ [34]. TFP consists of sending pulses that overlap strongly in time, frequency or in both, to maximize the SE, while introducing ICI and ISI. In the FSC transmitter encoding stage, low density parity check (LDPC) codes are introduced, to account for ISI, ICI and noise, while achieving the maximum information rate for a given modulation format [19], [35], [36]. The pulse shaping filters (typically electrical) are used to filter the data signal beyond Nyquist. At the receiver, coherent detection and DSP are employed. Due to the presence of ISI, TFP uses a sequence detector (like BahlCocke-Jelinek-Raviv (BCJR)), instead of a symbol-by-symbol detector used in other techniques. As an example, a 1.12 $\mathrm{Tb} / \mathrm{s}$ SBVT using TFP will consist of 7 FSC modules, each providing PM-QPSK $160 \mathrm{~Gb} / \mathrm{s}$ signals in $28 \mathrm{GHz}$ spectral bandwidth [37]. A typical spectrum for TFP is shown in the Fig. 5 (d). The code rate and SE are dependent on the optical signal to noise ratio (OSNR) of the link. Reach adaptation can be achieved using different code rates rather than different modulation formats.

\section{SDN control of optical transceivers}

The increase in the dynamicity of the networks and the need to respond quickly to changing requirements, has resulted in the operators and vendors embracing the SDN approach. The separation of the data and control planes and the centralization of the control operations, provide a path to a programmable network that can be dynamically provisioned to address the changing needs and requirements. By extending the SDN paradigm to the optical layer of the network and developing the control mechanisms for the optical hardware, such as transponders, a maximum network flexibility and reconfigurability can be achieved. Authors in [24] identify several transponder parameters that can be controlled, such as the line rate, modulation format, code, central frequency of the optical flows, and the association of optical transport network (OTN) streams with a specific optical flow. A review of several studies, related to the software defined optical networks $(\mathrm{SDON})$, can be found in [21]. Majority of researchers use the OpenFlow protocol, in order to control and configure the physical devices. In [35], the authors demonstrated an OpenFlow extension, which allowed for control of transmitter and receiver parameters, such as code rate and modulation format. Authors in [38], controlled a multi-flow, OFDM-based transponder, by adopting a REST-based application program interface (API). Typically, such studies assumed multi-flow transponders based on an array of lasers, where each laser is separately configured to meet the requirements of an optical flow. A first demonstration of SDN control of a frequency comb-based transponder has been reported in [39]. In that 
work, OpenFlow extensions have been proposed to control the radio frequency sources used in the frequency comb generation module, in order to set the spacing among optical carriers. In [40], [41], [42], [43], [44] authors investigated the virtualization of multi-flow transponder offering independent operations, control and management abilities to the clients or higher level network controllers. In particular, the demonstration of a virtualizable transponder has been reported in [42], while authors in [43] investigated the costs of virtualization. For more details and works on SDN control for optical networks, the reader can refer to the survey [21]. Recently, NETCONF [45] has emerging as an alternative SDN protocol for optical networks. Unlike OpenFlow, NETCONF is based on XML and YANG data modeling [46], which enables vendor-neutral description of network elements [47]. These features make NETCONF an attractive solution for operators and service providers. A lot of effort has been directed towards the development of YANG models that include features of multi-flow transponders (e.g., the OpenConfig project, activities within the Internet Engineering Task Force) [48], [49], [50]. The main focus of these efforts is to create a YANG models of the elastic network components and their attributes (e.g. modulation format, line rate for transmitters). Finally, an experimental demonstration integrating a single-flow transponder and NETCONF, has been reported in [51].

\section{Network Level Flexibility}

In addition to achieving transponder level flexibility, several critical network optimization operations are also related to transceiver characteristics. Examples of such operations are spectral de-fragmentation, restoration and traffic aggregation. The spectral fragmentation problem is similar to memory fragmentation in computer architecture. In a dynamic network scenario, connections are established and torn down randomly, resulting in a fragmented spectrum i.e. creation of spectral gaps. The fragmentation leads to inefficient use of spectral resources and affects network performance. Several spectral de-fragmentation techniques have been proposed and most of them involve transceiver reconfiguration. The push-pull technique [52] can perform de-fragmentation in a hitless fashion (i.e. without introducing additional errors) and involves the re-configuration of an allocated bandwidth and tuning of the central frequency of the carrier. The de-fragmentation speed can be increased if all the subcarriers in a superchannel provided by the SCG are frequency locked and can be tuned simultaneously [53]. Similarly, in a restoration process different frequency slots might be allocated on recovery paths resulting in the tuning of transmission signals. Traffic aggregation is another network optimization operation that requires a reconfigurable transmitter. In this case, traffic from several underutilized subcarriers can be aggregated, thus reducing the number of required subcarriers and releasing the valuable resources [54]. This operation requires control of the number of lines in the SCG.

\section{E. Subcarrier generator (SCG) features}

Based on the transceiver and network flexibility features described above, desired features of the subcarrier generation

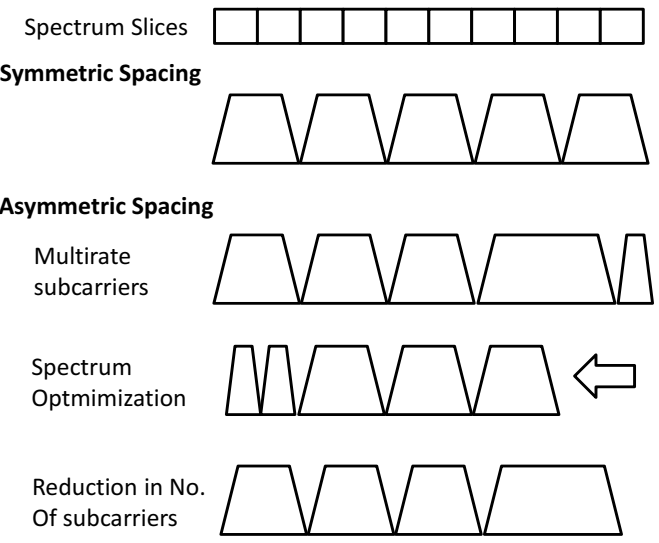

(a)

Figure 6: Different application scenarios for symmetric and asymmetric carrier spacing and line control.

module can be extracted. Most of the flexibility features are related to the characteristics of optical carriers and impose certain new requirements described below.

1) Standard laser features: The carrier source should have standard output power, high side mode suppression ratio (SMSR) and low phase noise. The optical characteristics of a laser have been defined in the optical internetworking forum specifications OIF-ITLA-MSA-01.3 [55].

2) High wavelength/frequency stability $O R$ frequency locked optical carriers: The optical subcarrier should also be highly stable in frequency in order to avoid ICI within a superchannel and reduce DSP processing requirements for carrier frequency estimation at the receiver. In other words, the carriers should be frequency locked with each other. High frequency stability can also improve the SE, by avoiding the spectral guard bands between the subcarriers within the superchannel.

3) Tunable carrier spacing: The bandwidth occupied by a subchannel is proportional to the baud rate. A superchannel that consists of identical subchannels will have equal carrier spacing, whilst the multi-rate superchannel and/or SBVT will have unequal carrier spacing. The carrier source should be able to provide both symmetric (equal) and asymmetric (unequal) tunable carrier spacing. Different scenarios for symmetric and asymmetric carrier spacing are shown in the Fig. 6 (a-e).

4) Concurrent wavelength tuning: As mentioned in earlier, simultaneous tunning of subcarriers allows to perform rapid network optimization, such as spectrum de-fragmentation and path restoration.

5) Control of the number of lines: This feature allows for switching any carrier ON/OFF as required. The carriers can be added to increase the overall information rate. Alternatively, the carriers can also be removed for operational efficiency (e.g. traffic aggregation, asymmetric subcarrier spacing) as shown in the Fig. 6 (e).

6) Spectral flatness: Just like conventional WDM networks, different optical carriers provided by a SCG should have equal output power for optimum transmission performance.

7) Fast tuning speed: The subcarriers should exhibit fast tunability, both individually and in groups. The overall setup 
Table I: THE RELATIONSHIP BETWEEN TRANSCEIVER AND NETWORK FLEXIBILITY AND SCG FEATURES.

\begin{tabular}{|c|c|c|c|}
\hline $\begin{array}{l}\text { Performance } \\
\text { Level }\end{array}$ & $\begin{array}{c}\text { Tuning } \\
\text { Parameter }\end{array}$ & $\begin{array}{l}\text { Dependency on } \\
\text { SCG? (Yes/No) }\end{array}$ & $\begin{array}{l}\text { Relevant SCG } \\
\text { Feature }\end{array}$ \\
\hline & $\begin{array}{l}\text { Modulation } \\
\text { Format }\end{array}$ & No & N/A \\
\hline \multirow[t]{5}{*}{ Transceiver } & Symbol Rate & Yes & $\begin{array}{l}\text { Tunable Carrier } \\
\text { Spacing, } \\
\text { Frequency } \\
\text { locking }\end{array}$ \\
\hline & Code Rate & No & N/A \\
\hline & $\begin{array}{c}\text { No. of } \\
\text { Subcarriers }\end{array}$ & Yes & $\begin{array}{c}\text { Control on } \\
\text { number of } \\
\text { lines/carriers }\end{array}$ \\
\hline & $\begin{array}{l}\text { Subcarrier } \\
\text { Spacing }\end{array}$ & Yes & $\begin{array}{c}\text { Tunable Carrier } \\
\text { Spacing }\end{array}$ \\
\hline & Slice-ability & Yes & $\begin{array}{c}\text { Asymmetric } \\
\text { Carrier Spacing }\end{array}$ \\
\hline \multirow[t]{2}{*}{ Network } & $\begin{array}{c}\text { Spectral } \\
\text { De-fragmentation }\end{array}$ & Yes & $\begin{array}{l}\text { Concurrent } \\
\text { Central } \\
\text { Wavelength } \\
\text { Tuning }\end{array}$ \\
\hline & $\begin{array}{c}\text { Traffic } \\
\text { Aggregation }\end{array}$ & Yes & $\begin{array}{l}\text { Control on } \\
\text { number of } \\
\text { lines/carriers }\end{array}$ \\
\hline
\end{tabular}

time should be low for lightpath provisioning, teardown or transponder re-configuration.

8) Phase coherence: This feature is not essential for standard implementations, but if available, can be useful in reducing the transceiver computational complexity. This aspect is explained in the next section.

Table I outlines how transceiver level and network level flexibility is related to features of the SCG. It appears that SBVT equipped with such an SCG can be instrumental for dynamic optical networking in next generation systems.

\section{LASERS VS. MW SOURCES BASED ON OPTICAL FREQUENCY COMBS IN A FLEX-GRID REGIME}

Standard telecommunication lasers are widely used in commercial WDM optical communication systems. However, the flex-grid networks need additional capabilities from an optical carrier source or SCG, as described above. In this context, OFC based sources have been proposed as an alternative to discrete or arrayed lasers. This section highlights problems associated with using the arrays of laser in the flex-grid regime and presents advantages that an OFC can offer. Some challenges/ disadvantages of OFCs are also briefly described. A detailed comparative analysis of different carrier source techniques will be reported in the forthcoming sections.

\section{A. Disadvantages of using discrete lasers in a flex-grid sce- nario}

In order to support the flexibility features envisioned for the multicarrier flex-grid transmission, the adjacent optical carriers should demonstrate correlation in certain optical characteristics, which are not found in an array of lasers. The performance aspects of a laser that are key in the flex-grid systems are described below.

1) Frequency drift: Lasers are subject to drift in the emission frequency over time. This drift can cause ICI in superchannels, which feature multiple, closely packed subcarriers. The spectral density of the frequency fluctuations of a semiconductor laser includes two main components: the high frequency component (approximately $1 \mathrm{MHz}$ to $2 \mathrm{GHz}$ ) and the low frequency component (up to $\sim 100 \mathrm{kHz}$ ). The high frequency component is due to the spontaneous emission and carrier density fluctuations within the laser cavity, causing spectral linewidth broadening. The low frequency component is caused by laser temperature fluctuations and acoustic perturbations, exhibiting $1 / f$ spectral distribution, and results in jitter of the laser spectrum. This in turn leads to the slow drift of the centre frequency of a laser [56]. In order to reduce the impact of the frequency drift, commercial transponders are equipped with wavelength lockers(WLs) / stabilizers. The most commonly used frequency stabilization technique is PoundDrever-Haul (PDH) method [57].

Integrated laser arrays can be used as an alternative to standard discrete lasers, lowering the cost, footprint and power consumption of the superchannel transmitters. Integrated multi-wavelength lockers that display up to $25 \mathrm{GHz}$ accuracy have been reported, reducing the number of WLs required [58], [59].

2) Fine-granularity wavelength locking: Tunable lasers can be tuned to any central frequency on the fixed grid within the $\mathrm{C}$-band, thus significantly reducing the inventory requirement. In the fixed grid scenario, channel centres are well defined and precisely spaced by $100 \mathrm{GHz}, 50 \mathrm{GHz}$ or in some cases $25 \mathrm{GHz}$. Tunable laser technology, which also includes the WL design and calibration, exploits this periodicity to simplify both hardware design and the calibration process [60]. However, in a flex-grid scenario, the frequency locations for the carriers are not strictly defined and the central frequency of subcarriers in a superchannel may not correspond to any frequency interval (even if $12.5 \mathrm{GHz}$ spacing is assumed). As a result, tunable lasers need to be able to tune to any frequency. Though such a fine-tuning granularity does not overly complicate the tuning control mechanisms of the laser itself, it does increase the complexity of the wavelength locking mechanism and the calibration process. WLs, designed for $50 \mathrm{GHz}$ fixed grid, provide a usable wavelength error feedback signal only within periodic regions around each of the $50 \mathrm{GHz}$ channel centre. Since no suitable feedback is available between these regions, the WL assembly for a flexgrid transmitter must be redesigned, to provide useful error feedback for all C-band frequencies. This is generally achieved by incorporating multiple WL elements, with complementary periodic spectral areas of coverage [60]. Recently, a new technique has been proposed, to compensate the frequency drift in high capacity superchannels, using the Han-Kobayashi coding technique [61]. In addition, authors in [62] proposed a laser wavelength stabilization technique using photonic mixing with the reference wavelengths and achieved an accuracy of 0.1 
GHz. The technique shifts the onus of accuracy from optical filters, typically used in conventional wavelength lockers, to microwave filters.

\section{B. Advantages and applications of MW sources in flex-grid networks}

Optical frequency combs can provide multiple frequency tones originating from a single laser. Typically, the tones are locked in frequency, symmetrically spaced and are coherent in phase. This broadband phase coherence, precise symmetrical tone spacing and faster tunability (central wavelength and carrier spacing) are distinguishing features of OFCs [10]. In the following, we describe the major advantages of OFCs over conventional lasers for applications in elastic/flexible networks.

1) Reduce the laser inventory, cost and power consumption: The introduction of superchannels, based on multiplexing of closely spaced carriers, will improve the spectral efficiency of a network, but result in the increase in the number of optical sources required. (S)BVT modules supporting flexgrid superchannels based on NWDM/CO-OFDM/TFP, would use an array of lasers both in the transmitter (source of optical carriers) and in the receiver, as local oscillator (LO). In addition to an array of lasers, an equal number of allied thermal and wavelength stabilization subsystems will also be needed. This will increase the cost, footprint and power consumption of carrier sources in the node/network.

Therefore, a compact optical source, capable of generating multiple optical frequencies/wavelength channels from a single device, could potentially simplify the operation and management of high capacity optical networks [24], [25], [63]. Optical frequency combs can provide the required number of frequency tones, hence have the potential to replace a large number of lasers and their allied control and stabilization circuitry.

2) Simpler and faster network reconfiguration: Elastic nodes can establish and tear down connections based on traffic dynamics and network operating conditions. The tuning speed of the components would thus determine the rate, at which the network provisioning, re-configuration and restoration can be carried out. In SBVTs equipped with coherent receivers, the overall setup time depends on the tuning time of the optical carrier, local oscillator and tunable filters. Commercially available narrow line-width lasers have a tuning time less than $10 \mathrm{msec}$, but there is a trade-off between the desired linewidth and tuning time [64], [65]. Superchannels are considered as a single manageable entity [28], whereas if the subcarriers originate from individual lasers, they have to be tuned one by one. As are result, the total setup time could be as long as $\left(N \times t_{L}\right)$, where $N$ is the number of subcarriers in the superchannel and $t_{L}$ is the tuning time for a single laser. Since the tones provided by the OFC are frequency locked with each other and can be concurrently tuned in wavelength by tuning the seed laser, the tuning time can be significantly reduced, without compromising the transmission quality. Concurrent tuning of frequency locked tones provided by OFCs would be vital for fast, hitless de-fragmentation as described in Table II and demonstrated in [66].
Table II: USE CASES FOR TUNABILITY: ARRAY OF LASERS AND VS. MW SOURCES.

\begin{tabular}{lll}
\hline \multicolumn{1}{c}{ Use case } & \multicolumn{1}{c}{ Array of Lasers } & \multicolumn{1}{c}{$\begin{array}{c}\text { Multi-wavelength } \\
\text { Sources }\end{array}$} \\
& $\begin{array}{l}\text { Tune each individual laser } \\
\text { De- } \\
\text { fragmentation by one, } \\
\text { Tuning time long, } \\
\text { No frequency locking, } \\
\text { Frequency error feedback } \\
\text { required for each laser to } \\
\text { ensure central frequency on } \\
\text { the grid }\end{array}$ & $\begin{array}{l}\text { Tune seed laser and } \\
\text { whole comb will tune } \\
\text { concurrently, }\end{array}$ \\
& $\begin{array}{l}\text { Fast tuning time, } \\
\text { Overall less setup time }\end{array}$ \\
\hline Changing Baud & $\begin{array}{l}\text { Step1:Tune each individual } \\
\text { rate }\end{array}$ & $\begin{array}{l}\text { Step1: Change the RF } \\
\text { laser one by one, } \\
\text { Tuning time long, }\end{array}$ \\
& Step2: Change the baud rate & $\begin{array}{l}\text { spacing of all the } \\
\text { subcarriers will change } \\
\text { at the same time. }\end{array}$ \\
& & $\begin{array}{l}\text { Step2: Change the baud } \\
\text { rate }\end{array}$ \\
& &
\end{tabular}

Similarly, OFCs can provide fast tuning of the subcarrier spacing in superchannels. In case of a laser array, a reconfiguration of the baud rate/subcarrier spacing, would require tuning of each laser individually. The line spacing in an OFC however, is mostly determined by the frequency of an electrical signal used for the comb generation. As a result, it can be easily changed, by tuning the RF signal frequency, as described in Table II. The typical re-configuration time for a RF synthesizer is $40-50 \mathrm{msec}$, however, for practical implementations, the reconfiguration time for the filter separating comb lines, must also be considered. The authors in [67] demonstrated a hitless reconfiguration of carrier spacing of a periodically poled lithium niobate (PPLN) based comb. The carrier spacing was tuned from $37.5 \mathrm{GHz}$ to $12.5 \mathrm{GHz}$ in $0.5 \mathrm{GHz}, 1 \mathrm{GHz}$ and $1.25 \mathrm{GHz}$ steps and a sweeping time was measured as $100 \mathrm{sec}, 80 \mathrm{sec}$ and $55 \mathrm{sec}$ respectively.

3) Enhanced spectral efficiency and reduced computational complexity: The improved frequency stability and phase coherence, inherently provided by the OFCs, are key features that could offer different benefits for different multiplexing/transmission techniques. Firstly, the frequency locked tones from an OFC, when used as carriers to construct a superchannel, can remove the need for guard bands, usually introduced to cater for laser frequency drift, thus improving the overall SE.

Secondly, the frequency stability and phase noise correlation of the optical carrier and LO are of critical importance to coherent link engineering, directly defining the link's performance and influencing the complexity of the DSP engine [68]. Due to a large frequency drift of an optical carrier (GHz-range), an optical coherent receiver does not use the conventional frequency/phase locked loops for tracking the signal-LO frequency offset and instead rely on DSP postprocessing techniques. The frequency offset estimation process is iterative and requires training sequences. Large frequency mismatch between the received signal and the LO may result in instabilities in the retrieval algorithm, leading to prolonged startup and/or data loss. In bidirectional transceivers, the 
frequency tracking becomes more complex, because the carrier is also used as a LO for reception [69].

In essence, large frequency and phase inaccuracies, lead to a high computational complexity and low energy efficiency of a coherent receiver. It is important to note that DSP processing is a major power consuming block in the coherent receiver [70]. Hence, the superior frequency stability and the phase coherence offered by OFC, can have a significant impact on the overall energy consumption of the optical network

4) Terabit coherent optical OFDM systems enabled by OFCs: With conventional implementations, the OFDM bandwidth is limited by the speed of the DAC. Since, the OFC consists of orthogonal and coherent comb lines, a wideband optical OFDM can be generated in the following two steps. 1) electro-optic generation of low speed optical OFDM signals on each comb line, 2) coherent and orthogonal multiplexing of OFDM modulated comb lines [71]. The architecture of a comb based Tb/s CO-OFDM is shown in Fig. 7. At the transmitter side, an OFC provides multiple comb lines with precise frequency spacing and optimum spectral flatness. The de-multiplexer separates comb lines, which are then modulated, using an IQ modulator, with low speed optical OFDM subcarriers, referred to as OFDM bands. All OFDM bands are then combined using a multiplexer. Using this method, a broad optical OFDM signal can be obtained, if the frequency spacing of the comb lines is an integral multiple of the OFDM subcarrier spacing. This feature is usually achieved by synchronizing the frequency of the comb generator and the OFDM modulators. However, multiplexers and de-multiplexers both in the transmitter and receiver should be gapless or strongly overlapping, allowing all spectral components to pass. All OFDM bands generated in this way, remain orthogonal to each other, due to the orthogonality of the comb lines. As a result, a much wider optical bandwidth OFDM signal is generated in comparison to when using state-of-the-art ADC/DAC and optoelectronics.

At the receiver, two de-multiplexers separate the incoming optical signal and the LO (comb lines) and direct them to the respective optical hybrids for coherent detection. In this architecture, the incoming signal is detected on per OFDM band basis [17] and the parallel coherent detection of multiband OFDM signals is performed using multiple comb lines as LOs. However, such parallel structure requires a large number of optical and electronic components. Thus optical integration of such receivers is essential to achieve a power and cost effective solution. The distinguishing feature of this receiver architecture is its ability to convert a very wideband optical signal to the electrical domain via optical spectrum slicing and electronic spectrum synthesis, as shown in the Fig. 7. From the figure it can be observed that each of the de-multiplexed OFDM band includes unwanted frequency components at the slice edges from the neighboring OFDM bands. In a carefully designed system, these frequency components are still orthogonal to the de-multiplexed OFDM band and cause no interference. The use of an OFC in place of multiple LOs guarantees that the phase relationship amongst optical spectral slices is preserved after $\mathrm{O} / \mathrm{E}$ conversion. Due to the preserved orthogonality and phase coherence, the DSP
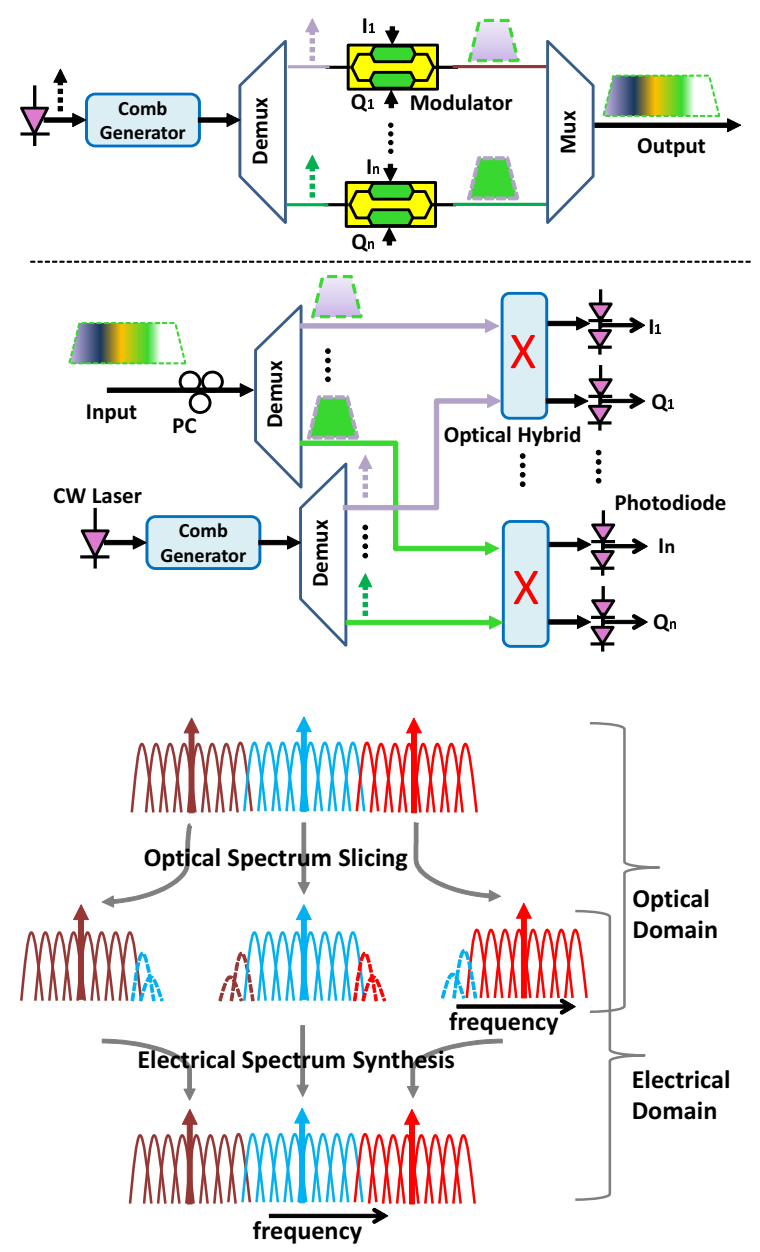

Figure 7: (top) Transmitter and receiver of $\mathrm{Tb} / \mathrm{s}$ coherent optical OFDM systems using optical frequency combs. (bottom) Optical-to-electrical conversion of three OFDM bands via optical spectrum slicing and electrical spectrum synthesis [71].

engine can remove the unwanted frequency components and subsequently synthesize the electrical spectrum, by stitching the spectra of multiple OFDM bands together.

5) Combs as multi-frequency local oscillator; A possible extension of previous scheme: In OFDM systems optical phase of multiple carriers must be estimated/controlled for spectrally efficient, interference-free transmission. In OFDM superchannels, transmitters have to oversample each channel to achieve Nyquist shape and in receivers additional bandwidth is required, in order to recover the adjacent channels carriers for the coherent crosstalk cancellation [72], [73]. In practice, these functions are implemented in a DSP engine. However, these signal processing functions not only waste the electrical bandwidth, but also restrict the throughput of each tributary channel, due to the low processing speed of DSP circuits. The authors in [74] proposed a filter-less multitone coherent orthogonal detection scheme, depicted in Fig. 8. Here, a multichannel reception of superchannel/OFDM signals is performed, using comb lines as a multi-frequency oscillator and exploiting their phase coherence and mutual 


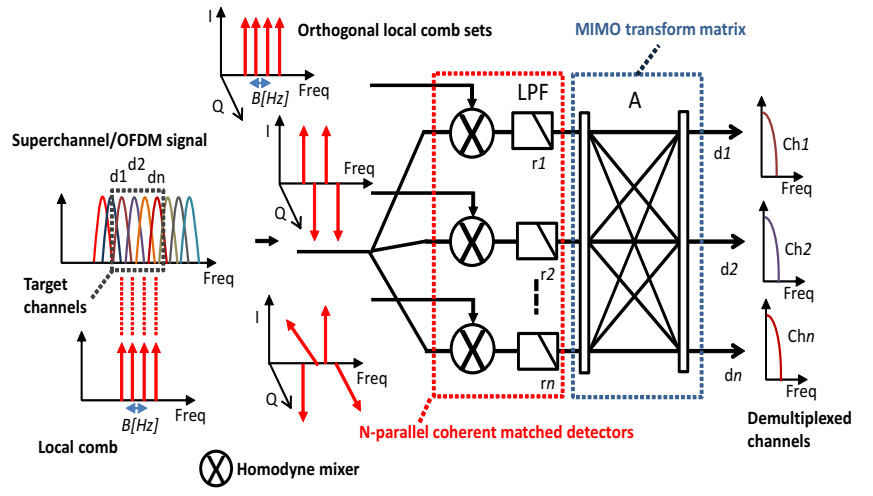

Figure 8: Comb as multi-frequency oscillator: Basic construction of multi-tone coherent matched detector for demultiplexing super-channel/OFDM signals [74].

orthogonality. The scheme overcomes the speed limitation, does not require additional electrical bandwidth and reduces the receiver complexity. The coherently matched detectors are placed in parallel configuration that effectively increase the optical bandwidth in the $100 \mathrm{~Gb} / \mathrm{s}-1 \mathrm{~Tb} / \mathrm{s}$ region beyond typical electrical bandwidth (10-40 Gb/s), recovering the optical phase relationship between the carriers in neighboring channels. Another advantage is that, unlike conventional de-multiplexing based on channel-by-channel separation and detection, the channels are separated and simultaneously detected, without the need of optical filters [74].

6) Role of optical frequency combs in Nyquist WDM transmission: As explained earlier, NWDM technique employs pulse shaping filters in the transmitter, to confine the bandwidth of the signal. RRC filters with a sharp roll off $(<0.2)$ are a popular choice to reduce the linear inter-channel crosstalk. In NWDM superchannels, multiple subcarriers are spaced at $1.2 \mathrm{x}$ symbol rate, to keep the amount of linear cross-talk below 0.5 dB OSNR [29], [32]. By using frequency locked carriers from OFCs, this guard band can be avoided, thus further increasing the spectral efficiency of the system.

7) Optical sinc-shaped Nyquist pulse generation: In the ideal Nyquist transmission, Nyquist pulses that carry the data symbols, overlap in the time domain with zero ISI. The ideal Nyquist filter that provides zero ISI, have a rectangular spectrum and a bandwidth equal to the symbol rate [64], [75]. High speed Nyquist signal generation is challenging, as the electrical pulse generation is restricted by the sampling rate and the bandwidth of DACs as well as the processing capabilities [76]. Super-flat optical frequency combs, with a rectangular spectrum and linear phase, can provide high quality Nyquist pulses [75]. Pulse parameters can be easily modified by tuning the OFC parameters, such as the comb bandwidth, the number of lines and their frequency spacing. In [75], Soto et al. demonstrated the generation of optical sinc-shaped Nyquist pulses of exceptional quality, using flat rectangular frequency combs, providing zero roll-off, minimum post-modulation spectral broadening and $1 \%$ deviation from ideal sinc shape. The authors in [76] demonstrated full band coherent detection of Nyquist pulses employing QPSK modulation and optical time division multiplexing (OTDM). Similarly, sinc-shape pulses can also be multiplexed in the frequency domain, following Nyquist WDM transmission scheme, where pulses can be generated and modulated for each carrier.

\section{Challenges of $M W$ sources}

While MW sources offer several advantages, over standard lasers, for dynamic optical networking, there is also a number of challenges, which must be addressed, if these sources are to be used within a commercial system.

1) Amplification stage: Since comb lines, in most cases, are sidebands of a seed signal, the overall power of the source of this signal (laser), is distributed among the lines. As a result, the power of an individual tone is lower than required for commercial systems. Therefore, an additional amplification stage, to bring the output power on par with discrete lasers, is required, resulting in an increased cost.

2) Limited carrier spacing: Unlike individual lasers that can be tuned to achieve an arbitrarily channel separation, the maximum achievable carrier spacing within a comb is limited $(<50 \mathrm{GHz})$. This is due to the electro-optic bandwidth constraints of devices involved in OFC generation. This OFC limitation can be addressed using devices having higher EO bandwidth.

3) Spectral contiguity: Spectral contiguity of comb lines (all the other carriers generated by an OFC are constrained to be used within a specific range of spectrum) can result in increased blocking probability during routing and spectrum assignment, thus restricting the network performance. Improved routing, spectrum and transponder assignment techniques have been recently proposed to improve the network performance in presence of MW sources [77].

4) Arbitrary asymmetric carrier spacing: Another challenge, associated with the use of MW sources, is how to achieve an arbitrary, asymmetric carrier spacing, from an OFC that inherently generates equally spaced tones. Several use cases for asymmetric carrier spacing have been depicted in the Fig. 6.

5) Tunable filtering/ de-multiplexing stage: In general, all tones generated by an OFC, are accessible from a single port. These lines must be routed to separate FSC modules for the data modulation, thus requiring an additional de-multiplexing or filtering stage. In the flex-grid networks, these filters must be tunable, to support disparate bandwidth needs. Designing tunable optical filters, with excellent optical characteristics and low complexity and cost, is a major challenge.

6) Long term stability: Long term stability conforming to industry standards is also one of the essential features. The optical characteristics such as flatness, SMSR etc. must be maintained.

7) Techno-economic feasibility: The commercial adoption of the MW sources is dependent not only on their technical advantages, but also the economic benefits. In order to successfully compete with the established technology, OFC-based transmitters need to achieve the footprint, cost and power consumption comparable or better than that of the existing solutions (i.e., standard discrete lasers and integrated laser arrays). 
In this section, we have presented the problems associated with using the individual laser sources in the flexgrid optical networks. We have also presented the advantages and main challenges in the application of OFC-based MW sources in flexible transceivers.

\section{Flexible Optical Frequency Combs as CARrier SOURCE IN OPTICAL NETWORKS}

An OFC can be simply defined as a source that consists of a series of equally spaced discrete spectral lines. The tree diagram in Fig. 9 depicts different types of OFC generators for application in optical communications. Majority of the techniques are based on different types of electro-optic (EO) modulators. Other comb generation techniques employ micro ring resonators (MRR), PPLN waveguides and gain switched lasers.

In this section we will review, compare and contrast these comb generation techniques, with a focus on their application in elastic optical networks. The techniques have been discussed in the same order as presented in the Fig. 9.

\section{A. Electro-optic optical frequency comb generators}

The general scheme of electro-optic comb generation is based on modulating continuous wave (CW) light. This can be achieved using one or more modulators that operate on the amplitude, phase, polarization or combinations thereof, of a $\mathrm{CW}$ signal. The modulators are driven by RF signal oscillators, which create multiple sidebands, leading to generation of an OFC at the output of the optical system. The scheme is highly flexible; frequency spacing between the comb lines (free spectral range - FSR) can be varied by tuning the RF drive signal and the entire comb spectrum can be concurrently tuned by adjusting the emission frequency of the $\mathrm{CW}$ laser. The number of comb lines can be increased by cascading modulators. A flat spectrum can be obtained by the appropriate selection of the characteristics of modulators and driving signals. The phase noise properties of these comb tones are mainly determined by the seed CW laser [78]. Despite being simple and flexible, the application of this type of OFC's in optical communications were limited due to technical constraints (such as inherent bias drift, high insertion loss etc.). With advances in RF oscillator (low noise high frequency) and modulator technologies (low $V_{\pi}$, high electrical power handling), this OFC generation technique has attracted a renewed interest [10]. Fig. 10 shows the relationship between the output comb characteristics, the operating conditions and the properties of related devices in the comb generation system. In electro-optic OFC generators, the modulation index $\Delta \Theta=\pi V / V_{\pi}$ defines the overall comb bandwidth, where $\mathrm{V}$ is applied RF voltage and $V_{\pi}$ is half-wave voltage (required for a $\pi$ phase shift). $V_{\pi}$ depends on material properties and waveguide structure of the modulator. The modulation index can be maximized by designing modulators with a low $V_{\pi}$ and high RF power handling capabilities. As mentioned earlier, further increase in the comb bandwidth (or number of lines) can be achieved either by adding more modulators in tandem or by creating fiber ring cavities, containing modulators in the loop (e.g. simple phase modulator or re-circulating frequency shifter). Comb expansion will be discussed in detail in the next subsections.

The upper limit on the FSR is dependent on the electrical bandwidth of the modulators. In addition to Lithium Niobate $\left(\mathrm{LiNbO}_{3}\right)$ and Indium Phosphide (InP), silicon organic hybrid $(\mathrm{SOH})$ modulators with low $V_{\pi}$ have also been used for comb generation [78], [79]. The typical bandwidth of commercially available $\left(\mathrm{LiNbO}_{3}\right)$ modulators is $20 \mathrm{GHz}-40 \mathrm{GHz}$. However, high bandwidth (up to $100 \mathrm{GHz})\left(\mathrm{LiNbO}_{3}\right)$ [80], polymer [81] and $\mathrm{SOH}$ [82] modulators have also been reported recently.

The intensity of each of the side mode created by EO modulation is governed by Bessel functions and highly depends on the harmonic order. This issue makes the generation of OFCs with optimum spectral flatness (small amplitude ripple) a challenging task [83]. The appropriate selection of modulator driving conditions is mandatory for a flat spectrum. Obtaining a flat OFC spectrum is not a straightforward because the system is inherently nonlinear and the power distribution between the comb lines is not uniform, when using a driving RF signal composed of a single frequency [10]. In recent years, extensive efforts have been made by researchers to achieve flattop $(<1 \mathrm{~dB}$ amplitude ripple) EO OFC spectrum. Another distinguishing feature of electro-optic modulators is that the most of designs can be photonically integrated, which is essential for future high bit rate flexible transceivers. In summary, EO modulator based combs satisfy most of the vital requirements of a flexible carrier source to be employed in future high capacity elastic transponders.

In the following, different EO OFC generation techniques reported will be explained in chronological order. For the sake of clarity, the EO combs have been divided into two major categories: combs using single and/or cascaded modulators and coupled cavity combs. The former category includes OFCs involving same and/or different types of modulators.

1) EO Combs based on intensity modulator (IM) and phase modulator (PM) in tandem: As briefly discussed earlier, OFC generators employing a single modulator may have a limited spectral bandwidth. Additionally, the spectral flatness will be poor, unless the bias and RF drive signals are carefully chosen. The most popular alternative solution to achieve a flat spectrum is to use intensity and phase modulators in tandem [84], as depicted in Fig. 11 (a). When biased appropriately, the intensity modulator produces a train of pseudo-square pulses . These pulses carve out the portion of the $\mathrm{CW}$ light, where the chirping provided by the phase modulation is almost linear, thus resulting in an equalized output spectrum [10]. The modulation index of the phase modulator defines the resultant comb bandwidth and can be increased by a factor of $N$ by adding $N$ additional phase modulators. The proper alignment of temporal phases in time between the modulators is critical for spectral flatness and can be achieved with phase shifters shown in the schematic. If the electric field of the input light is expressed as $E_{i n}(t)=\exp \left(i w_{c} t\right)$, the electrical field of the output is $E_{\text {out }}(t)$ expressed as [13]:

$$
E_{\text {out }}(t)=\exp \left(i \omega_{c} t\right) \cos \left(\frac{\pi}{4} \alpha \sin \omega_{m} t-\frac{\pi}{2} \gamma\right)
$$




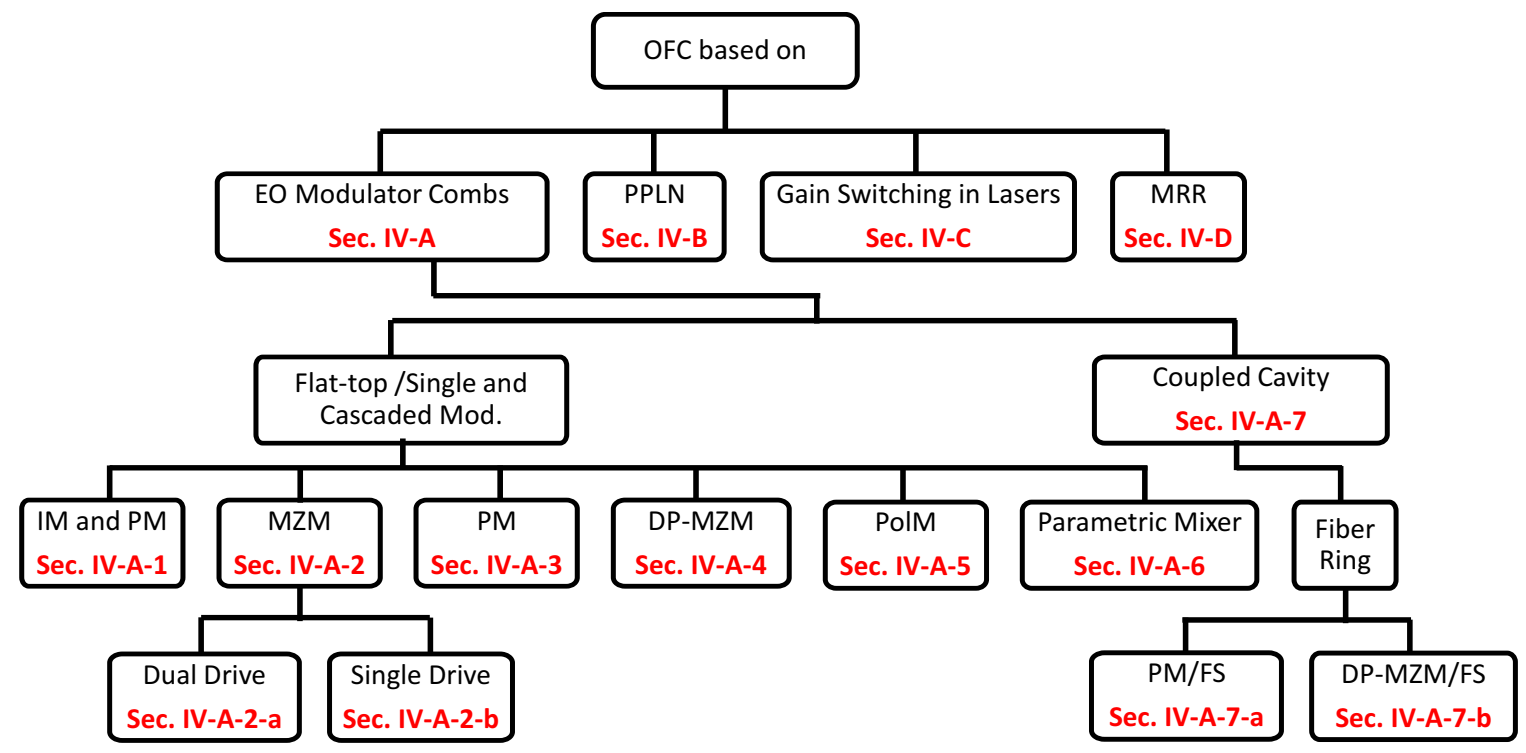

Figure 9: Optical frequency comb generation techniques for optical communications.

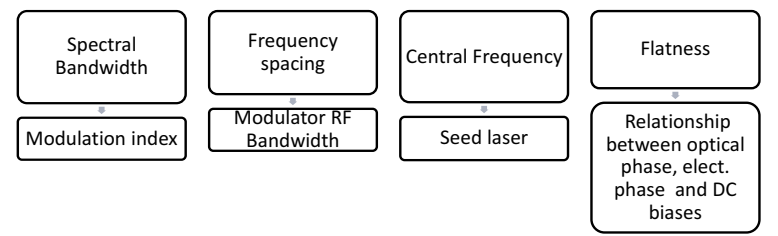

Figure 10: Comb flexibility parameters and related devices and their properties.

$$
\times \exp \left\{i\left(\theta \frac{\pi}{4} \alpha+\frac{\pi}{2} \beta\right) \sin \omega_{m} t-i \theta \frac{\pi}{2} \gamma\right\}
$$

where; $\omega_{m}$ : the angular frequency of the sinusoidal electrical signal, $\alpha$ and $\beta$ : electrical voltages normalized by the halfwave drive voltage $\left(V_{\pi}\right)$ of the IM and PM respectively, $\gamma(0 \leq$ $\gamma \leq 1)$ : the dc bias voltage normalized by $\left(V_{\pi}\right)$ of the IM to set transmittance. Number of spectral lines and desired level of flatness of the output comb depends on values of $\alpha, \beta$ and $\gamma$. Usually, three dimensional contour plots are generated to determine optimum values of $\beta$ and $\gamma$ for a fixed value of $\alpha$, for the desired number of lines and spectral flatness.

An 8 line OFC with a FSR of $12.5 \mathrm{GHz}$ and $2 \mathrm{~dB}$ spectral flatness was demonstrated using a cascaded IM and PM [84]. Subsequently, the authors extended their work and proposed an optical carrier supply module (OCSM) [89] (consisting of comb, amplifiers and de-multiplexer/filters) and demonstrated $1.28 \mathrm{~Tb} / \mathrm{s}(512 \times 2.5 \mathrm{~Gb} / \mathrm{s})$ super-dense WDM transmission over $320 \mathrm{~km}$, covering the $\mathrm{C}$ and L bands. A total of 64 seed lasers were used to generate 512 carriers using two OCSMs. The work was followed by building a prototype providing, the evidence of how combs could be used in real nodes as a carrier source[13], [90]. Mao et al. further investigated the crosstalk and the practical system design, using comb based carrier sources [91]. Although the proposed prototype was targeted towards low bit rate $(2.5 \mathrm{~Gb} / \mathrm{s})$ systems, the considerations of practical system design can be extended for future high capacity terabit elastic nodes. In this survey, we will describe node and network level considerations in a flex-grid terabit scenario in the latter sections.

In [92], an integrated $\left(\mathrm{LiNbO}_{3}\right)$ OFC source designed and built by Fujitsu and employing a customized design of an IM and a PM as a single device, was presented. The generated comb displayed an overall spectral bandwidth of $7 \mathrm{~nm}$ at a FSR of $10 \mathrm{GHz}$ and a spectral flatness of less than $10 \mathrm{~dB}$. Low drive voltage modulators $\left(1.3 \mathrm{~V} V_{\pi}\right.$ for PM and $2.3 \mathrm{~V}$ for IM @ 10 $\mathrm{GHz}$ ) were realized by optimizing the design of electrodes and using a long PM with a $149 \mathrm{~mm}$ folded waveguide. This resulted in a improved modulation index and a larger spectral bandwidth of the output comb. Another approach, depicted in Fig. 11 (b), is the OFC based on an overdriven cascaded DDMZM and PM that generated 23 lines at $25 \mathrm{GHz}$ spacing and $1.6 \mathrm{~dB}$ spectral flatness [85]. This entailed a DD-MZM being driven by fundamental signal followed by a PM, was driven by the second harmonic.

Torres-Company et al. provided theoretical analysis and a novel generalized approach to understanding the shape of optical frequency combs, using the notion of time-to-frequency mapping [86]. A user-defined envelope of optical frequency combs can be achieved following a two-step process as shown in Fig. 11 (c). "First, the target spectral profile is tailored on the temporal envelope of a pulse train. In a second stage, another device introduces pure quadratic phase modulation on each pulse, also known as time lens. If the amount of chirp is sufficiently large, the optical spectral envelope will be a scaled replica of the tailored intensity pulse" [10]. It implies that for a flat frequency comb we need a flat-top pulse train and a pure quadratic phase modulation on each pulse to translate temporal intensity shape into spectral domain i.e., time-tofrequency mapping [86]. The pulse train can be generated by intensity modulating a $\mathrm{CW}$ laser and then introducing a quadratic phase modulation using a PM. A 19 line comb at $20 \mathrm{GHz}$ spacing and approximately $1 \mathrm{~dB}$ spectral flatness was generated numerically as shown in Fig. 11(d). However, 

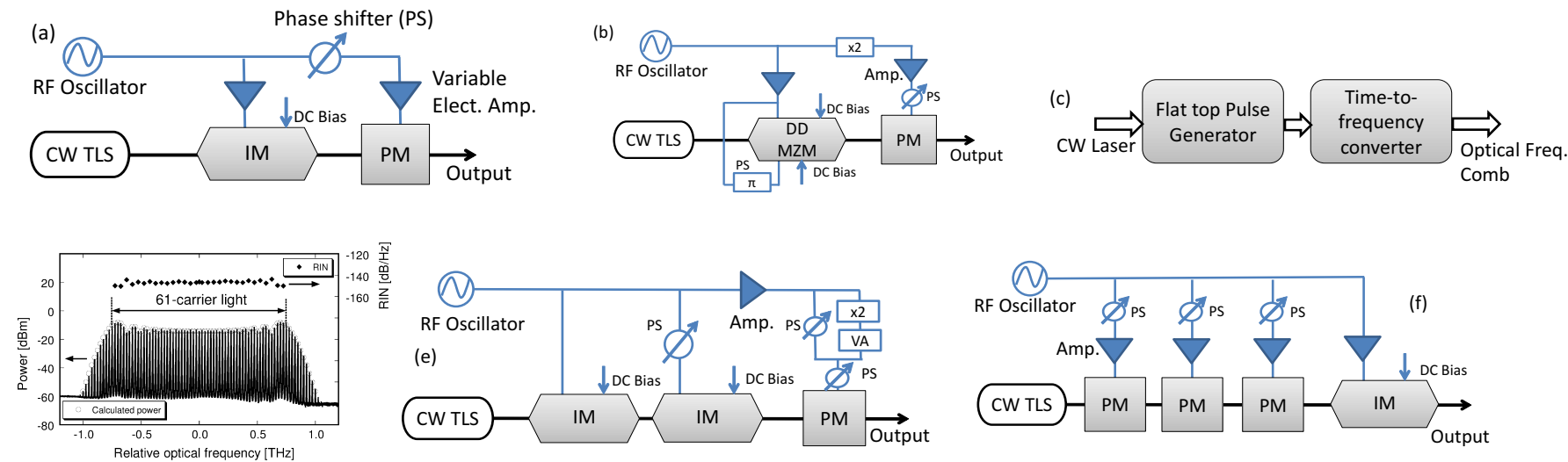

Figure 11: Combs based on cascaded IM and PM (a) Basic scheme [84] (b) DD-MZM and PM in tandem [85] (c,d) Theoretical model for flatness and resultant comb [86] (e) Cascaded comb employing tailored RF drive signal [87] (f) Cascaded scheme supporting high input power and broader output spectrum [88].

the spectrum was not flat at the edges, which appeared as a "rabbit-ear" or "bat-ear-like" structure, due to the deviation of phase modulation from a pure quadratic, within the pulse duration. An improvement to this solution was achieved by making the flat top pulse much steeper (using two IMs) and by tailoring the RF signal driving the phase modulator (to improve the the quadratic profile) [87], as shown in Fig. 11(e). The sharper time domain waveform reduces the fraction of the signal that can drift from the desired quadratic temporal phase. The tailored RF waveform is generated by mixing the first and the second harmonic with a suitable amplitude ratio $\left[\left(\frac{1}{16}\right)^{2} \sim-24 d B\right]$ and phase shift $\left(180^{\circ}\right)$, in order to suppress the fourth order harmonic. Higher order harmonics are less of an issue, as the cascaded IMs create a waveform with a sharper decay. The output comb had 38 lines spaced at $10 \mathrm{GHz}$, with less than $1 \mathrm{~dB}$ spectral flatness. This proved that the flatness can be improved by either linearizing the chirp, by reducing the temporal duration of the flat-top pulses or both [87].

An alternative solution proposes that the spectral flatness of an OFC is related to the direct current (DC) bias of the IM and the optimum ratio of DC bias to half-wave voltage driving the IM. The optimum ratio was found to be 0.35 [93]. The output comb had the spectral flatness of $<1 \mathrm{~dB}$, whilst generating 15 lines at a FSR of $10 \mathrm{GHz}$. The number of lines can be increased by increasing the phase modulation index, just like other cascaded modulator schemes. Interestingly, the scheme only uses ordinary sinusoidal modulating signal and does not require any additional tailored RF waveform to drive the PM or a dispersive medium between the modulators, to achieve an ultra-flat OFC. The parabolic function can be replaced by a cosine function.

Another realisation of a tunable OFC based on cascaded modulators was reported in [88]. This solution uses PMs in the first stage instead of an IM, as shown in the Fig. 11 (f) and has a high input power $(\sim 1 \mathrm{~W})$ handling capability.

Terabit coherent OFDM systems enabled by OFCs based on cascaded intensity and phase modulators, both at the transmitter and receiver, have also been successfully experimentally demonstrated, proving their feasibility for future elastic networks [71]. Some other efforts, focusing on energy efficiency [94] and a comparative analysis of the cascaded PM and IM scheme with other cascaded modulators techniques (in terms of spectral purity and stability) have also been reported [95]. Table III summarizes the evolution of this technique highlighting the problems addressed and the contribution to the improvements.

2) Combs based on Mach-Zehnder Modulators (MZM); Single and Cascaded: Both single drive and dual drive MZMs have been used in different configurations to generate optical frequency combs.

a) Dual Drive Modulator based combs: Another technique to realize a flexible and flat top OFC involves the use of dual drive Mach-Zehnder modulators (DD-MZM). The earliest OFC based on $\left(\mathrm{LiNbO}_{3}\right)$ DD-MZM proposed by Veselka et al. [96], [97] involved amplitude modulation of the CW light followed by the use of Kerr nonlinearities in nonlinear fiber, as shown in Fig. 12 (a). The externally modulated light is amplified and coupled to a nonlinear dispersion shifted fibre (DSF), with an intensity dependent refractive index. Here it generates higher order spectral sidebands due to self-phase modulation (SPM). Furthermore, an OFC using a single DDMZM was investigated theoretically in 2007 [83], as a less complex and cost effective alternative to cascaded modulator schemes. In this scheme, the DD-MZM is asymmetrically driven by a sinusoidal signals having different amplitudes on both arms, as shown in the Fig. 12 (b). The spectra obtained from phase modulation of the upper and lower arms are non-flat. However, with appropriate settings of the relative optical and RF phases, both spectra, when recombined at the output of the DD-MZM, become complementary and can provide a relatively flat optical frequency comb [10]. The theoretical analysis provided analytical formulas for " $f$ lat spectrum condition" and "maximum efficiency condition" for such schemes. The flat spectrum condition is given by [83]:

$$
\Delta A \pm \Delta \theta=\pi / 2
$$

where $\Delta \theta=\theta 1-\theta 2 / 2$ is the difference between optical phase delays in the two arms corresponding to the dc bias difference. $\Delta A=(A 1-A 2) / 2$ is given by the difference in drive 
Table III: OPTICAL FREQUENCY COMBS BASED ON CASCADED IM AND PM

\begin{tabular}{|c|c|c|c|c|c|c|c|}
\hline $\begin{array}{l}\text { Sr. } \\
\text { No. }\end{array}$ & Ref. & Year & Target/ Issue Addressed & $\begin{array}{l}\text { No. of } \\
\text { Lines }\end{array}$ & $\begin{array}{l}\text { Carrier } \\
\text { Spacing }\end{array}$ & Flatness & $\begin{array}{l}\text { Application/ } \\
\text { Demonstration }\end{array}$ \\
\hline 1 & [84] & 2001 & $\begin{array}{l}\text { Flattened comb } \\
\text { generation }\end{array}$ & 8 & $12.5 \mathrm{GHz}$ & $2 \mathrm{~dB}$ & Comb only \\
\hline 2 & [89] & 2002 & $\begin{array}{l}\text { Proposed optical carrier } \\
\text { supply module (OCSM) }\end{array}$ & 8 & $12.5 \mathrm{GHz}$ & $2 \mathrm{~dB}$ & $\begin{array}{l}1.28 \mathrm{~Tb} / \mathrm{s}(512 \times 2.5 \mathrm{~Gb} / \mathrm{s}) \\
\text { super-DWDM transmission }\end{array}$ \\
\hline 3 & {$[13]$} & 2003 & $\begin{array}{l}\text { OCSM prototype } \\
\text { demonstration }\end{array}$ & 8 & $12.5 \mathrm{GHz}$ & $2 \mathrm{~dB}$ & A complete system level prototype \\
\hline 4 & [91] & 2004 & Line increase & 31 & $4 \mathrm{GHz}$ & $3 \mathrm{~dB}$ & $\begin{array}{l}2.5 \mathrm{~Gb} / \mathrm{s} \text { transmission, comb } \\
\text { wavelength tuning }\end{array}$ \\
\hline 5 & [92] & 2006 & Integrated comb source & 87 & $10 \mathrm{GHz}$ & $8 \mathrm{~dB}$ & Experimental characterization \\
\hline 6 & [90] & 2006 & Terresterial transmission & 8 & $12.5 \mathrm{GHz}$ & $2 \mathrm{~dB}$ & Terresterial transmission \\
\hline 7 & [86] & 2008 & Flatness & 19 & $20 \mathrm{GHz}$ & $\sim 1 \mathrm{~dB}$ & Numerical solution \\
\hline 8 & [85] & 2009 & Carrier spacing & 23 & $25 \mathrm{GHz}$ & $1.6 \mathrm{~dB}$ & $\begin{array}{l}\text { Experimental transmission } \\
\text { characterization }\end{array}$ \\
\hline 9 & [87] & 2010 & Flatness & 38 & $10 \mathrm{GHz}$ & $<1 \mathrm{~dB}$ & Experimental demonstration \\
\hline 10 & [71] & 2010 & $\begin{array}{l}\text { Comb enabled } \\
\text { transmission }\end{array}$ & 32 & $10 \mathrm{GHz}$ & $<5 \mathrm{~dB}$ & $\begin{array}{l}1.008 \mathrm{~Tb} / \mathrm{s} \mathrm{CO}-\mathrm{OFDM} \text { system } \\
\text { demonstration, combs as carrier } \\
\text { source in Tx and LO in Rx }\end{array}$ \\
\hline 11 & [93] & 2011 & Flatness & 15 & $10 \mathrm{GHz}$ & $<1 \mathrm{~dB}$ & Comb only \\
\hline 12 & [95] & 2013 & Spectral purity, stability & 23 & $12.5 \mathrm{GHz}$ & & $\begin{array}{l}\text { Experimental comparison in terms } \\
\text { of spectral purity and stability }\end{array}$ \\
\hline 13 & [88] & 2013 & $\begin{array}{l}\text { Stand alone flexible comb } \\
\text { source }\end{array}$ & $60-75$ & $6-18 \mathrm{GHz}$ & $10 \mathrm{~dB}$ & $\begin{array}{l}\text { A complete discrete prototype, } \\
\text { High input power handling }(1 \mathrm{~W})\end{array}$ \\
\hline 14 & [94] & 2013 & Energy efficiency & 9 & $4 \mathrm{GHz}$ & $\begin{array}{l}1 \text { and } 3 \\
\mathrm{~dB}\end{array}$ & Comb experimental demonstration \\
\hline
\end{tabular}

voltages applied to the two RF electrodes and corresponds to the phase difference induced in each arm. The optimal driving condition to obtain a flat spectrum with maximum conversion efficiency, termed as "maximum efficiency condition", is expressed as [83]:

$$
\Delta A=\Delta \theta=\pi / 4
$$

The analysis also provides an estimation of the generated comb bandwidth i.e., expected number of lines and is given by [78], [83]:

$$
N \sim \pi^{2} V_{p p} / 2 V_{\pi}
$$

These theoretical and simulation results were experimentally validated [98] generating 11 and 19 lines at $10 \mathrm{GHz}$ spacing with $1.1 \mathrm{~dB}$ and $4.3 \mathrm{~dB}$ amplitude variation/ripple. We can observe that the flatness is comparable with the cascaded IM and PM scheme, but this technique requires high RF drive power in order to achieve a higher modulation index. E.g., 35.9 $\mathrm{dBm}$ and $36.4 \mathrm{dBm} \mathrm{RF}$ signals are required for a modulator with a $V_{\pi}=5.4 \mathrm{~V}$, in order to produce $19 \mathrm{comb}$ lines [78]. Two approaches have been pursued to reduce the RF drive power requirements namely:

i) optimization of RF signals

ii) employ alternative materials in order to achieve low $V_{\pi}$ modulators.

On the first approach, Mishra et al. simulated the effect of using different RF drive signals, in order to find an optimum solution for generating an OFC with less drive power, but with the same spectral flatness and number of lines [99]. In subsequent work [100], the authors demonstrated generation of a 9 line $\mathrm{OFC}$, exhibiting a $1.1 \mathrm{~dB}$ of spectral flatness and a $6.25 \mathrm{GHz}$ frequency spacing, using a single DD-MZM $\left(V_{\pi}=3.5 \mathrm{~V}\right)$, driven by $1-1.6 V_{\pi}$ amplitude RF signals. The main distinction from previous work is that one arm is driven by the fundamental frequency, while the other by the second harmonic, generated by a frequency doubler, as shown in the Fig. 12 (c). The scheme benefits from the superposition of optical subcarriers generated by the fundamental and its second harmonic and can provide variable number of lines.

The second approach to reducing the power requirements of the OFC generation, requires the use of modulators built with alternative materials. These include InP [78], [101], [102] and $\mathrm{SOH}$ [79], which have been recently demonstrated using state of the art photonic integration techniques. A $9 \times 12.5 \mathrm{GHz}$ line optical comb was generated using an InP based DDMZM with $4.3 \mathrm{~dB}$ flatness [102] and less than $1 \mathrm{~dB}$ [101] spectral flatness. Slavik et al. demonstrated a compact optical comb generator using an InP tunable laser integrated with a push-pull modulator [78]. The comb generated using this approach with RF drive powers of $27 \mathrm{dBm}$, provided 29 tones with an amplitude ripple $<3 \mathrm{~dB}$. Hence, this technique yields $50 \%$ more lines, while using $10 \mathrm{~dB}$ less $\mathrm{RF}$ drive power than in [98], clearly showing the advantages of the InP platform [83]. A slightly different approach (optimizing driving conditions) entailed a reduction in the $V_{\pi}$ of the modulator and was achieved by biasing the modulator higher near the bandgap 

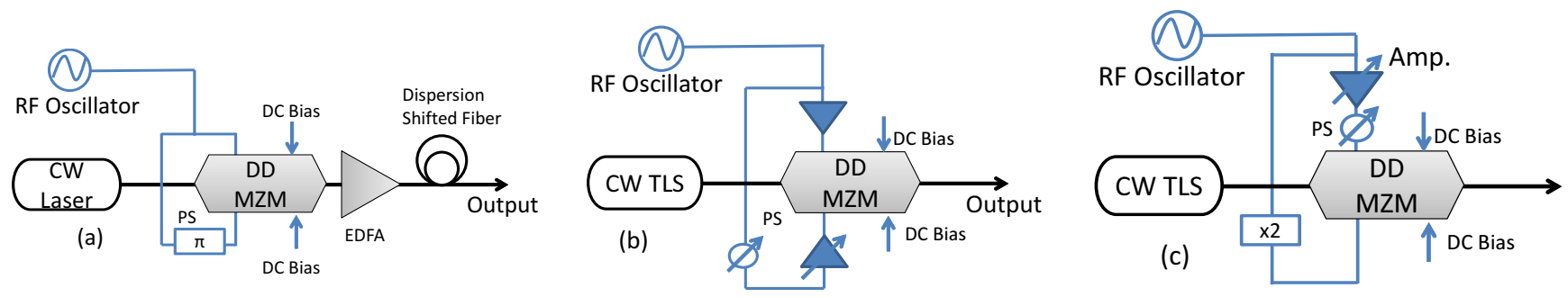

Figure 12: Combs based on DD-MZM (a) DD-MZM and self-phase modulation in dispersion shifted fiber [96] (b) Asymmetrically driven DD-MZM [83] (c) Scheme driving DD-MZM using fundamental and second harmonic signals [100].

for deeper modulation, compromising on residual amplitude modulation. Without biasing near the bandgap, the modulator would have produced similar number of lines as for $\mathrm{LiNbO}_{3}$. Weimann et al. demonstrated an integrated OFC source based on an SOH electro-optic DD-MZM. It is the first modulatorbased comb source on a silicon-on insulator (SOI) substrate, which is suitable for monolithic integration and has low $V_{\pi}$. OFCs exhibiting 9 lines at FSR of $25 \mathrm{GHz}$ and 7 lines at a FSR of $40 \mathrm{GHz}$, both with a spectral flatness of $2 \mathrm{~dB}$, were generated. The OFC was employed in a communication system transmitting an aggregate data rate of $1 \mathrm{~Tb} / \mathrm{s}$ over $300 \mathrm{~km}$ of standard single mode fibre (SSMF). However, it is important to note that such OFCs display high insertion losses ( $21 \mathrm{~dB})$ [79]. DD-MZM based OFCs have also been used as transmitters and as filter-less multi-frequency LOs in multitone coherent matched detection system. A multi-frequency LO enables simultaneous and orthogonal multi-channel demultiplexing/demodulation of super-channel OFDM signals without the need for additional electrical bandwidth [74].

Hraghi et al. [103] proposed a modified simulated annealing-based optimization method to find the necessary ranges, for which the DD-MZM based comb source is ultra-flat (less than $1 \mathrm{~dB}$ ripple) for different number of lines. A modified flatness condition was proposed, involving two additional parameters: maximum allowed amplitude ripple/flatness and number of lines. It is vital to note that modulator based OFCs are sensitive to variations in bias and changes in RF signal phase and power. Botia et al. [104] have used fuzzy logic to predict stability and found that changes to comb flatness are more often caused by variations in RF power, rather than changes in the DC bias. Such findings can be very useful in flexible operations of combs, where we want to change the number of lines or frequency spacing. Table IV summarizes the recent works based on this technique.

b) Combs employing single drive intensity modulators (IM): Conventional single drive MZMs have also been used to generate flexible OFCs. Both single [76] and multiple MZMs in cascade [105] have been used, driven by single or multiple frequency RF signals. A summary of recent relevant reports can be found in Table V. Similar to other EO modulator based techniques, the use of multiple devices can enhance the comb bandwidth. However, number of lines for the larger carrier spacing are limited (approximately 9) compared to cascaded IM and PM schemes. OFCs produced by IMs have better rectangular shape as they exhibit excellent flatness $(<1$ $\mathrm{dB}$ ) and a high SMSR. Ellis and Gunning in [106] proposed an OFC using two IMs in cascade, shown in Fig. 13 (a) and exploited the phase coherence among the comb lines to demonstrate a spectrally efficient $40 \mathrm{~Gb} / \mathrm{s}$ CoWDM system [106]. Authors in [107] reported an OFC based on repeated frequency shifting using two MZMs and an asymmetric Mach Zehnder interferometer (AMZI), as shown in Fig. 13 (b). The scheme was proposed as an alternative to frequency shifter loops based on fibers, for improved stability, by avoiding need of precise resonant conditions. The achievable carrier spacing corresponds to the FSR of the AMZI used. The stability of such an OFC is dependent on the stability of the AMZI and the bias drift of MZMs. Any instability in an AMZI can lead to variations in comb carrier spacing and subsequently to the generation of undesired sidebands. The generated comb exhibited 7 lines spaced at $26.8 \mathrm{GHz}$, but with a poor flatness $(\sim 20 \mathrm{~dB})$.

A bit rate flexible OFDM transceiver, using a single zerochirp IM based comb, was experimentally demonstrated in [108]. The flexible comb, shown in Fig. 13 (c), enabled a transceiver to achieve flexible bit rate, by varying the number of lines (1 to 5) and subcarrier spacing. However, the scheme used two fixed RF clock sources to change carrier spacing. Larger carrier spacing can be obtained by photonic RF synthesis, avoiding use of multiple clocks and/or frequency multipliers [109], [110]. Imran et al. proposed a modified solution (Fig. 13 (d)) for flexible comb with larger flexible carrier spacing (12.5 GHz-100 GHz) and variable number of lines (1-5), by using photonic RF synthesis with just one additional laser and a photo-detector. The line-width of the generated lines will be slightly higher than seed laser, because two independent lasers have been used for RF signal generation, limiting its application to metro networks. The technique in [109] provides desired features of flexible OFC, but is complex and relies on the use of additional MLLs and DFBs for photonic RF synthesis. As such it is not viable for telecommunication applications, where the OFCs are meant to reduce the number of lasers.

Frequency locked carriers from an OFC have also been used to demonstrate hitless spectral de-fragmentation in flex-grid networks [53], [66]. $400 \mathrm{~Gb} / \mathrm{s}$ QPSK superchannel consisting of $4 \times 25 \mathrm{GHz}$ subcarriers from cascaded IMs based OFC was tuned for spectral de-fragmentation through the control plane.

Quite a few variants of the cascaded configuration, aimed at different comb quality aspects, have also been reported in the literature. In [105] different operating modes for the first IM (minimum or maximum transmission) are exploited to 
Table IV: OPTICAL FREQUENCY COMBS BASED ON DUAL DRIVE MZM

\begin{tabular}{|c|c|c|c|c|c|c|c|}
\hline $\begin{array}{l}\text { Sr. } \\
\text { No. }\end{array}$ & Ref. & Year & Target/ Issue Addressed & $\begin{array}{l}\text { No. of } \\
\text { Lines }\end{array}$ & $\begin{array}{l}\text { Carrier } \\
\text { Spacing }\end{array}$ & Flatness & $\begin{array}{l}\text { Application/ } \\
\text { Demonstration }\end{array}$ \\
\hline 1 & [96] & 1997 & $\begin{array}{l}\text { Early work, comb based on DD } \\
\text { MZM and kerr NL }\end{array}$ & 7,11 & $32 \mathrm{GHz}$ & $3 \mathrm{~dB}, 10 \mathrm{~dB}$ & Transmission characterization \\
\hline 2 & [97] & 1998 & Lines, Carrier spacing & 13 & $64 \mathrm{GHz}$ & $10 \mathrm{~dB}$ & Transmission characterization \\
\hline 3 & [83] & 2007 & $\begin{array}{l}\text { Theoretical model for DD MZM } \\
\text { combs }\end{array}$ & N/A & N/A & N/A & Siumulation, Analytical formulae \\
\hline 4 & [98] & 2007 & $\begin{array}{l}\text { Complexity, flatness, conversion } \\
\text { efficiency }\end{array}$ & 11,19 & $10 \mathrm{GHz}$ & $\begin{array}{l}1.1 \mathrm{~dB}, 4.3 \\
\mathrm{~dB}\end{array}$ & $\begin{array}{l}\text { Experimental demonstration of } \\
\text { [83], } \mathrm{LiNbO}_{3} \text { device }\end{array}$ \\
\hline 5 & [99] & 2011 & Flatness, Performance comparison & 8 & $12.5 \mathrm{GHz}$ & $<1 \mathrm{~dB}$ & $\begin{array}{l}\text { OFDM implementation based on } \\
\text { combs }\end{array}$ \\
\hline 6 & [102] & 2013 & Integrated InP comb & 9 & $12.5 \mathrm{GHz}$ & $4.9 \mathrm{~dB}$ & Tuning over $\mathrm{C}$ band demonstrated \\
\hline 7 & [101] & 2013 & $\begin{array}{l}\text { OFC using asymmetrically driven } \\
\text { InP DD MZM }\end{array}$ & 9 & $12.5 \mathrm{GHz}$ & $<1 \mathrm{~dB}$ & $\begin{array}{l}\text { Theoretical analysis and } \\
\text { experimental demonstration }\end{array}$ \\
\hline 8 & [100] & 2013 & Cost and energy efficiency & $\begin{array}{l}3,4 \\
5,9\end{array}$ & $6.25 \mathrm{GHz}$ & $\leq 1.32 \mathrm{~dB}$ & $\begin{array}{l}\text { Tunable comb, Uses double the } \\
\text { RF frequency in one arm }\end{array}$ \\
\hline 9 & [104] & 2013 & $\begin{array}{l}\text { Stability, flatness, optimization in } \\
\text { dynamic scenario }\end{array}$ & N/A & N/A & N/A & $\begin{array}{l}\text { Evaluates effect of variables (RF } \\
\text { power, bias, phase) on stability } \\
\text { using fuzzy logic }\end{array}$ \\
\hline 10 & [74] & 2013 & $\begin{array}{l}\text { Comb enabled transmission and } \\
\text { filter-less multichannel reception } \\
\text { in coherent receiver }\end{array}$ & 23 & $10 \mathrm{GHz}$ & $4.3 \mathrm{~dB}$ & $\begin{array}{l}\text { OFC as source in Tx and as } \\
\text { filter-less multi-frequency LO in } \\
\text { multi-tone coherent matched } \\
\text { detection system. }\end{array}$ \\
\hline 11 & [103] & 2014 & Flatness, Optimization & N/A & N/A & N/A & $\begin{array}{l}\text { Numerical modeling, Optimal } \\
\text { driving conditions for given lines } \\
\text { and flatness }\end{array}$ \\
\hline 12 & [79] & 2014 & $\begin{array}{l}\text { Integrated comb sources based on } \\
\text { SOH DD MZM }\end{array}$ & 7,9 & $\begin{array}{l}25 \mathrm{GHz}, 40 \\
\mathrm{GHz}\end{array}$ & $2 \mathrm{~dB}$ & $\begin{array}{l}\text { Monolithic Si integration. } \mathrm{Tb} / \mathrm{s} \\
\text { transmission }\end{array}$ \\
\hline 13 & [78] & 2015 & $\begin{array}{l}\text { Integrated (InP) OFC, fully } \\
\text { integrated laser and modulator }\end{array}$ & 29 & $10 \mathrm{GHz}$ & $3 \mathrm{~dB}$ & $\begin{array}{l}\text { Experimental demonstration, Max. } \\
\text { lines using DD MZM }\end{array}$ \\
\hline
\end{tabular}

produce variable number of lines. When an IM is biased at the minimum or maximum transmission, even or odd harmonics are generated, which are fed to second stage, producing a different number of lines. Yuhua et al. demonstrated an OFC having frequency spacing twice the RF frequency [111]. Authors in [112] proposed a theoretical model for an OFC based on the chirping of MZMs. Here, the second modulator is driven by a delayed replica of first RF signal. Simulation analysis shows that higher number of lines can be produced using this method, although no experimental validation is provided and practical device constraints, such as modulator bandwidth, has not been considered. Similarly, a $20 \times 10 \mathrm{GHz}$ OFC, having $0.6 \mathrm{~dB}$ spectral flatness and employing two IMs and a DP-MZM modulator in cascade, depicted in Fig. 13 (e), was demonstrated in [113]. IM based combs (Fig. 13 (f)) have also been used to generate sinc pulses for NWDM/OTDM systems, due to rectangular spectral profile of the comb [75], [76], [114]. Sinc pulses, with pulse widths ranging from 3.5 ps to 8.75 ps, repetition rates from 52.5 ps to 157 ps and duty cycles from $2.2 \%$ up to $16.6 \%$, were experimentally generated.

3) Combs based on phase modulators (PM): The principle of generating sidebands using phase modulators is the same as for other electro-optic modulators. Phase modulators stand out from their IM counterpart due to low insertion losses, smaller size and for not requiring DC bias controllers to maintain stable operation. In addition, PM based combs provide higher tone-to-noise ratio (TNR, also referred to as effective OSNR). The early efforts of OFC generation, based on a single PM driven by sinusoidal modulating signals (Fig. 14 (a)) provided good performance, but limited achievable bandwidth [115], [116].

Another design consisting of two PMs, with a dispersive medium in between, achieving a larger number of lines and improved stability, is shown in Fig. 14 (b) [117]. The output of first PM and dispersive medium is equivalent to IM providing flat spectral profile. As a result, the design is equivalent to a cascaded IM and PM scheme. The dispersive medium between the modulators, can be realised using either fibers or chirped fiber Bragg gratings (CFBG). CFBG are more resistant to temporal fluctuations caused by temperature change, thereby provide better stability. Reduced timing fluctuations (1/200) of the second phase modulator, also helps to stabilize the entire spectrum of the comb. The optical spectrum after the second PM is given by [117]:

$$
P_{k, 2 n d P M}=P_{C W}\left|\sum_{n=-\infty}^{\infty}\left[J_{n}\left(\Delta \theta_{1}\right) J_{k-n}\left(\Delta \theta_{2}\right) \times \operatorname{expi}\left(\varnothing_{n}+\varnothing_{d i f}\right)\right]\right|^{2}
$$

where $\theta_{1}, \theta_{2}$ are modulation indices of PM1 and PM2 and $\phi_{n}$ denotes the phase change of the nth modulation sideband caused by the dispersion. The relative timing difference be- 

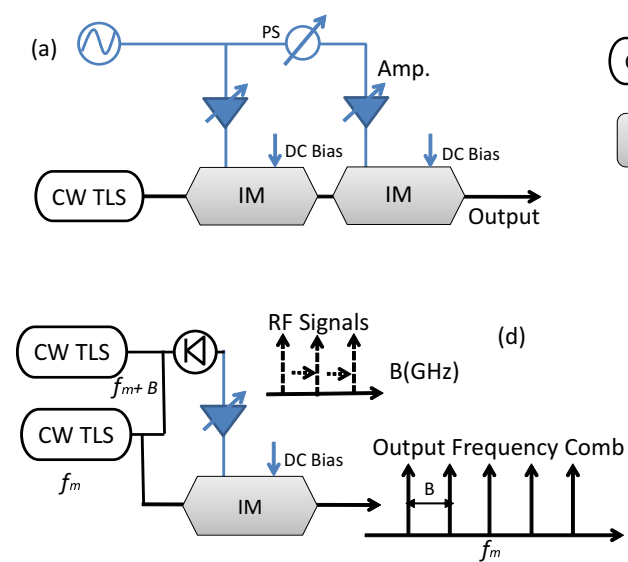
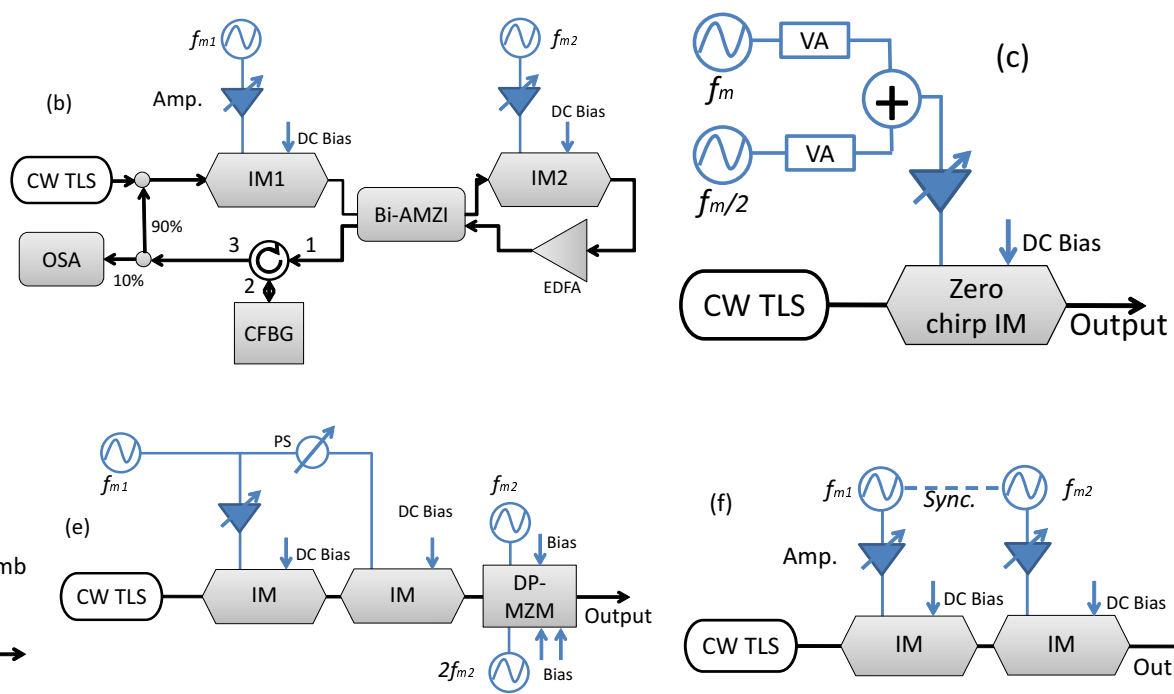

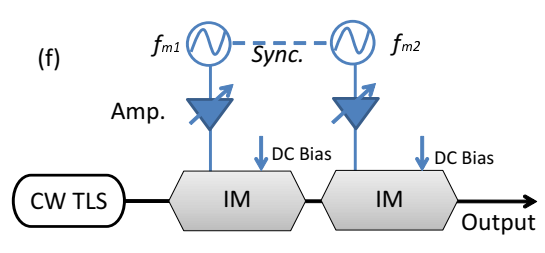

Figure 13: OFCs based on single drive MZM (a) Using two MZMs in cascade (b) Scheme using two MZMs and bi-directional AMZI for frequency shifting [107] (c) Flexible OFC for flexible OFDM transceiver [108] (d) Flexible carrier source using photonic RF synthesis [110] (e) Cascaded IM and DPMZM [113] (f) Scheme for Sinc pulse generation [75].
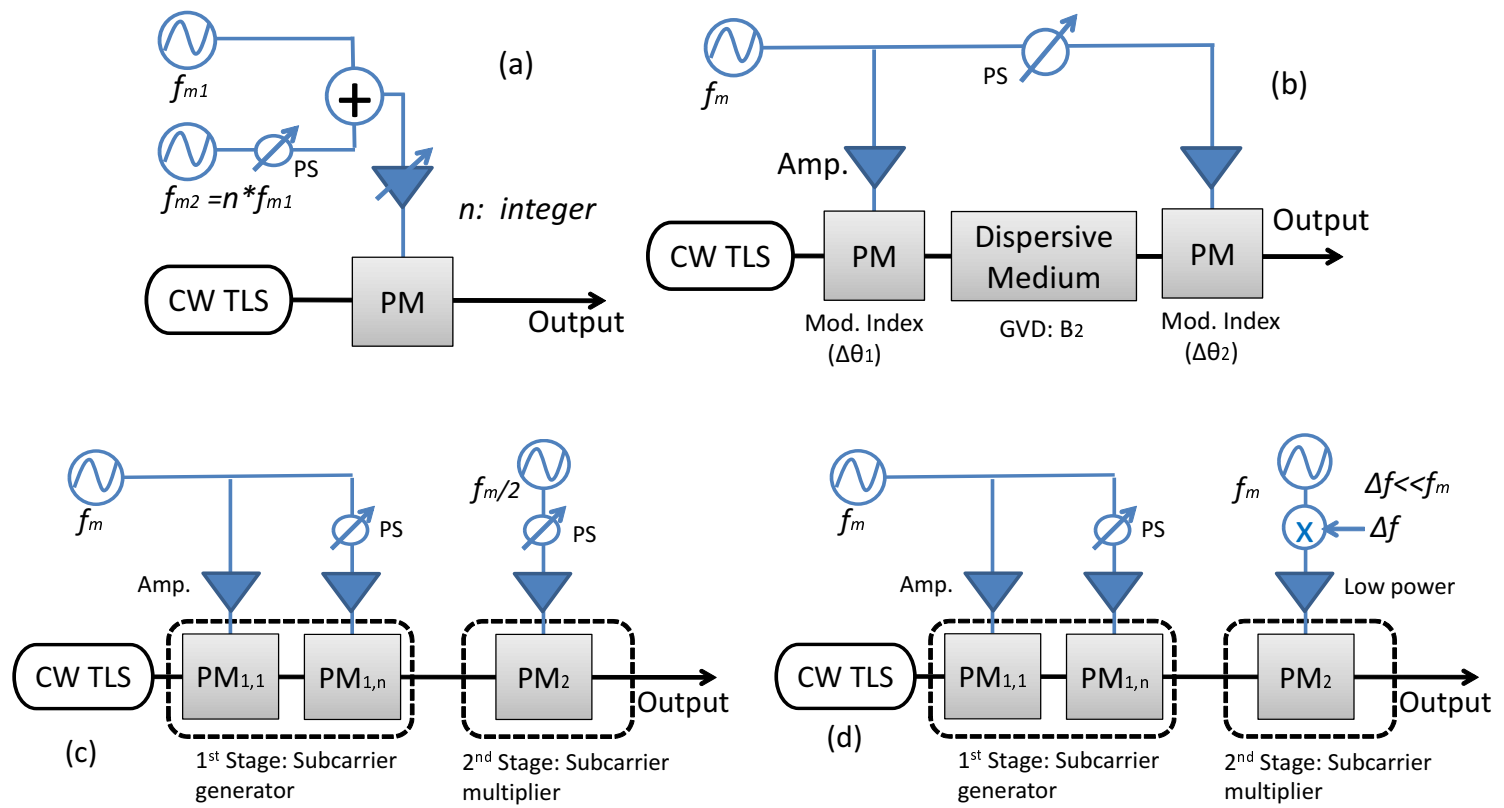

Figure 14: Combs based on phase modulators (a) Comb using single PM [115] (b) Comb using cascaded PMs with a dispersive medium in the middle [117] scheme (c) Two stage cascaded PMs without dispersive medium[118] (d) Optimized two stage scheme using single high frequency RF clock [119].

tween first and second PM, $t_{d i f}$ is:

$$
t_{\text {dif }}=N / 2 \mathrm{fm}
$$

and $\phi_{\text {dif }}$ is the resulting phase change of each sideband, caused by the timing difference of the modulation. $N$ is an arbitrary integer. A flat spectrum can be obtained if the following conditions for the modulation index and group velocity dispersion (GVD) $B_{2}$ of the dispersive medium are met:

$$
\Delta \theta_{1}=\pi / 4
$$

and

$$
B_{2}= \pm 1 / 4 f_{m}^{2}
$$

In addition, authors in [117] also found an approximate relationship between comb lines and second stage PM modulation index $\theta_{2}$.

$$
\Delta \theta_{2}=0.48 N_{\text {carrier }}-0.11
$$

If the conditions above are met, the spectral bandwidth of the OFC can be increased by increasing $\theta_{2}$. A complete system level OFC prototype demonstration, showing 61 lines 
Table V: OPTICAL FREQUENCY COMBS BASED ON SINGLE DRIVE MZM

\begin{tabular}{|c|c|c|c|c|c|c|c|}
\hline Sr. No. & Ref. & Year & Target/ Issue Addressed & $\begin{array}{l}\text { No. of } \\
\text { Lines }\end{array}$ & $\begin{array}{l}\text { Carrier } \\
\text { Spacing }\end{array}$ & Flatness & $\begin{array}{l}\text { Application/ } \\
\text { Demonstration }\end{array}$ \\
\hline 1 & [106] & 2005 & $\begin{array}{l}\text { Interferometric crosstalk control } \\
\text { in transmitter using phase } \\
\text { locked comb lines }\end{array}$ & 5,9 & $40 \mathrm{GHz}$ & $\begin{array}{l}0.1 \mathrm{~dB}, 1.2 \\
\mathrm{~dB}\end{array}$ & $\begin{array}{l}\text { SE enhancement using CoWDM } \\
\text { technique based on OFC }\end{array}$ \\
\hline 2 & [107] & 2009 & Stability & 7 & $26.8 \mathrm{GHz}$ & $\sim 20 \mathrm{~dB}$ & $\begin{array}{l}\text { Stable OFC using two MZMs and } \\
\text { AMZI instead of DP-MZM }\end{array}$ \\
\hline 3 & [108] & 2009 & $\begin{array}{l}\text { OFC enabled transceiver } \\
\text { flexibility }\end{array}$ & $1,2,3,5$ & $10 \mathrm{GHz}$ & $\sim 3 \mathrm{~dB}$ & $\begin{array}{l}\text { Experimental demons. of OFDM } \\
\text { BVT based on flexible OFC }\end{array}$ \\
\hline 4 & [66] & 2013 & $\begin{array}{l}\text { Comb enabled network } \\
\text { flexibility }\end{array}$ & 4 & $12.5 \mathrm{GHz}$ & $1 \mathrm{~dB}$ & $\begin{array}{l}\text { Hitless spectral de-fragmentation } \\
\text { of } 400 \mathrm{~Gb} / \mathrm{s} \text { signals using } \\
\text { cascaded IM comb }\end{array}$ \\
\hline 5 & [75] & 2013 & $\begin{array}{l}\text { Sinc shaped Nyquist pulse } \\
\text { generation }\end{array}$ & 9 & Variable & $1 \mathrm{~dB}$ & $\begin{array}{l}\text { Sinc pulse generation for } \\
\text { OTDM/NWDM systems using } \\
\text { rectangular shaped IM combs. }\end{array}$ \\
\hline 8 & [105] & 2014 & $\begin{array}{l}\text { Tunable comb bandwidth, } \\
\text { Number of lines }\end{array}$ & 15,20 & $10 \mathrm{GHz}$ & $<1 \mathrm{~dB}$ & $\begin{array}{l}\text { Experimental flexible comb based } \\
\text { on cascaded IM }\end{array}$ \\
\hline 9 & [111] & 2014 & $\begin{array}{l}\text { Carrier Spacing, } \\
\text { Comb using cascaded IMs }\end{array}$ & 4,5 & - & - & $\begin{array}{l}\text { Flexible OFC having carrier } \\
\text { spacing twice the modulation } \\
\text { frequency }\end{array}$ \\
\hline 10 & [113] & 2014 & $\begin{array}{l}\text { Energy efficiency, low driving } \\
\text { power required }\end{array}$ & 20 & $10 \mathrm{GHz}$ & $0.6 \mathrm{~dB}$ & $\begin{array}{l}\text { Comb only, Cascaded two IMs } \\
\text { and one DP-MZM }\end{array}$ \\
\hline 11 & [112] & 2015 & $\begin{array}{l}\text { Comb based on chirping } \\
\text { cascaded MZM }\end{array}$ & 27 & $5-40 \mathrm{GHz}$ & $<1 \mathrm{~dB}$ & $\begin{array}{l}\text { Simulation only, Uses a single RF } \\
\text { source }\end{array}$ \\
\hline 12 & [53] & 2015 & Comb enabled elastic operations & 4 & $25 \mathrm{GHz}$ & $\sim 1 \mathrm{~dB}$ & $\begin{array}{l}\text { Spectral defragmentation using } \\
\text { comb controlled by PCE control } \\
\text { plan }\end{array}$ \\
\hline
\end{tabular}

with a FSR of $25 \mathrm{GHz}$ and displaying $8 \mathrm{~dB}$ spectral flatness, can also be found in [117]. An improved approach for PM combs is to cascade PMs in two stages with two different phase modulation indices and without any additional dispersive medium. Two stages of sinusoidal phase modulations at different modulation depths are achieved by dividing PMs in two groups, as shown in the Fig. 14 (c) [118]. The first stage is called subcarrier generator and second stage is called subcarrier multiplier (doubler). Zhang et al. proposed a flat spectrum scheme, where two stage phase modulation was achieved by driving the two PM groups with different RF modulation frequencies and different modulation depths [118]. The flattened peak power of the phase-modulated optical subcarriers is achieved by optimizing the phases of the sinusoidal driving signals. The optimal phase differences and modulation index for two stage PMs was obtained theoretically and is given by [118]

$$
\Delta \varphi=\frac{(2 n+1)}{4} \pi \quad(n=0, \pm 1, \pm 2, \ldots)
$$

53 spectral lines spaced by $12.5 \mathrm{GHz}$, having TNR $>40 \mathrm{~dB}$ and less than $10 \mathrm{~dB}$ amplitude ripple, were generated experimentally. The modulators were driven by fundamental and second harmonic signals, which means such schemes require two clock sources or an additional RF multiplier/divider. Later, the authors improved their work and achieved two stage phase modulation by using a single high frequency RF source as shown in Fig. 14 (d). A small frequency offset signal (500 $\mathrm{kHz}$ offset for $25 \mathrm{GHz}$ ) was introduced in the second stage to obtain the second stage phase modulation [119]. A 21 line phase-insensitive, stable and flattened comb, with an FSR of $12.5 \mathrm{GHz}$ and a $3 \mathrm{~dB}$ spectral flatness, was successfully demonstrated. The transmission performance of the spectral lines was also evaluated in a $160 \mathrm{~Gb} / \mathrm{s}$ PM-QPSK system. DP-QPSK/ DP-8QAM terabit all optical AO-OFDM superchannel flexible transmitter [120] and DP-16QAM/DP-8QAM 
terabit Nyquist superchannel flexible transmitter, employing PM based combs, have also been tested in field trials over long haul networks. However, fluctuations in transmission performance for some subcarriers were observed mainly caused by the intensity difference between neighboring subcarriers (i.e., non-ideal flatness) and also the OSNR distribution. The intensity fluctuations were minimized to maintain the spectrum flatness by using an automatic control loop on WSS that separates subcarriers for onward data modulation. Highest capacity, capacity-distance product (at the time of publishing) and enhanced reach by use of adaptive modulation format and/or code rate was also demonstrated. Successful field trials prove that optical frequency combs can be used in future terabit transceivers, if cost-effective designs are commercially available.

4) Optical frequency combs based on nested MZM/IQ modulator : Nested MZM, sometimes also referred to as dual parallel MZM (DP-MZM) or IQ modulators, are compact integrated devices providing six control parameters for optimization. Since two sub-MZMs are integrated on a single chip, this method of OFC generation does not suffer from synchronization and phase matching issues, unlike other multiple modulators based schemes [122]. In addition, being a single device, the IQ modulator avoids the use of multiple RF clock sources. The fundamental scheme for the generation of an OFC using an IQ modulator is depicted in Fig. 15 (a).

The input optical signal from the seed laser is modulated by RF signals applied to both sub-MZMs. An OFC is generated in each of the sub-MZMs, where the phase and amplitude of the harmonics are determined by the applied RF drive voltage and modulator bias [126]. These two sub-MZM outputs then interfere at the output port of the DP-MZM. The interference is controlled by the bias of the outer MZM and the relative RF phase difference to generate a spectrally flat OFC. Different number of lines and frequency spacing can be obtained under different operating conditions. The six controllable parameters are the biases of the three MZMs, two drive voltages and the relative phase between them. Gheroma et al. proposed and demonstrated the first OFC using DP-MZM. A general expression for the relative output power in the sideband (comb line) is given by [122]:

$$
\begin{gathered}
4 I_{k}=J_{k}^{2}\left(\Delta \Phi_{1}\right) \cos ^{2}\left(\frac{\Theta_{1}}{2}+k \frac{\pi}{2}\right)+J_{k}^{2}\left(\Delta \Phi_{2}\right) \times \cos ^{2}\left(\frac{\Theta_{2}}{2}+k \frac{\pi}{2}\right)+ \\
2 J_{k}\left(\Delta \Phi_{1}\right) J_{k}\left(\Delta \Phi_{2}\right) \times \cos \left(\frac{\Theta_{1}}{2}+k \frac{\pi}{2}\right) \cos \left(\frac{\Theta_{2}}{2}+k \frac{\pi}{2}\right) \cos (k \varphi+\Psi)
\end{gathered}
$$

Here $\Theta_{1}, \Theta_{2}$ are sub-MZM bias angles, $\psi$ is bias angle of outer MZM, $\varphi$ is the RF phase difference the two inputs, $\Delta \Phi i=(\pi / 2)\left(V i / V_{\pi}\right)$ is the phase modulation index of subMZMs, with $V_{i}$ applied voltage and $V_{\pi}$ half wave voltage at the drive frequency.

O'Riordan et al. [122] carried out a detailed simulationbased analysis to find the possible number of lines and frequency spacing under different operating conditions of the six controllable parameters. The authors also demonstrated that with the appropriate selection of parameters, asymmetric harmonic generation can also be achieved. In such case, the carrier frequency is no longer at the center of the comb spectrum. Different types of combs, having 2 lines at FSR of 30 and $60 \mathrm{GHz}, 3$ lines at $30 \mathrm{GHz}, 4$ lines at 10 and $20 \mathrm{GHz}$ and 7 lines at $10 \mathrm{GHz}$ frequency spacing with less than $1 \mathrm{~dB}$ spectral flatness, were generated experimentally. Some combinations presented in the simulation analysis and requiring high drive voltages, can also be obtained, if MZMs with a low $V_{\pi}$ were employed in the DP-MZM. This means that a higher number of lines can be generated by improving the modulation index of the MZMs, similar to other EO modulator based OFC techniques.

Authors in [123] proposed a slightly modified scheme, allowing for an improved SMSR. Here, one arm of the DPMZM was driven by double the frequency with respect to the other arm, as shown in the Fig. 15 (b). A flexible number of flat comb lines, with a power variation less than $3 \mathrm{~dB}$, the SMSR higher than $15 \mathrm{~dB}$ and a tunable FSR, were generated. A Gaussian-shaped OFC with low drive voltages was also demonstrated.

DP-MZM based OFCs have also been used for Nyquist pulse generation [127]. The repetition rate of generated Nyquist pulses can be changed by tuning the frequency of the RF source. Pulses with a timing jitter of less than 258 fs and a signal to noise ratio of more than $30 \mathrm{~dB}$ were generated. Wang et al. proposed to to generate an OFC by driving only one arm of DP-MZM, as depicted in Fig. 15 (c) [124]. This design reduces the need for multiple synchronized RF sources, phase shifters and frequency multipliers and eliminates the requirement for the RF power balancing.

Nested MZM have also been widely used as a frequency shifter (FS) or single side band suppressed carrier (SSB-SC) modulator [128], [129], [130]. OFCs using a nested MZM as a FS have been rigorously investigated. Stability, high extinction ratio (ER) and SMSR are essential for a good quality FS, but electro-optic modulators suffer from bias drifting issues, which in turn affect the ER and SMSR. High values of ER and SMSR have been achieved in [131] by improving the bias stability through the use of an electronic feedback stabilization loop. It is easier to control the bias point drift of the modulator, when the bias is set to maximum, minimum or quadrature point and that can significantly simplify the practical implementation [126]. A high ER integrated DPMZM was realized in [132] by introducing an active Y-branch in both sub MZMs. Recently, integrated SOH FS have also been reported [133], demonstrating a $10 \mathrm{GHz}$ shift using 1 $\mathrm{mm}$ phase modulators. The authors also proposed a concept of temporal shaping of the drive signal, in order to overcome the trade-off between the SMSR and conversion efficiency. OFCs using DP-MZM as a FS in a re-circulating amplified loop fall into coupled cavity frequency combs category and will be discussed separately in the paper. Sambo et al. demonstrated the first software defined network (SDN) controlled SBVT and its sliceability feature, using a discrete programmable carrier source based on multiple FSs in a parallel configuration [39]. Later, the authors provided a complete stand-alone characterization and transmission performance evaluation (420 $\mathrm{Gb} / \mathrm{s}$ using TFP) of this source, named few wavelength source 
Table VI: OPTICAL FREQUENCY COMBS BASED ON SINGLE AND CASCADED PHASE MODULATORS

\begin{tabular}{|c|c|c|c|c|c|c|c|}
\hline Sr. No. & Ref. & Year & Target/ Issue Addressed & $\begin{array}{l}\text { No. of } \\
\text { Lines }\end{array}$ & $\begin{array}{l}\text { Carrier } \\
\text { Spacing }\end{array}$ & Flatness & $\begin{array}{l}\text { Application/ Technique } \\
\text { improvement }\end{array}$ \\
\hline 1 & [116] & 2005 & $\begin{array}{l}\text { Early work, Comb based on } \\
\text { single PM }\end{array}$ & $17-29$ & -- & -- & $\begin{array}{l}\text { Comb alone, PM driven by } \\
\text { fundamental and harmonic } \\
\text { signals }\end{array}$ \\
\hline 2 & [115] & 2008 & $\begin{array}{l}\text { Early work, Comb based on } \\
\text { single PM }\end{array}$ & 9,11 & $3 \mathrm{GHz}$ & $\begin{array}{l}0.8 \mathrm{~dB} \\
1.9 \mathrm{~dB}\end{array}$ & $\begin{array}{l}\text { Comb only, PM driven by dual } \\
\text { sine wave }\end{array}$ \\
\hline 3 & [117] & 2009 & $\begin{array}{l}\text { Line increase and stability } \\
\text { improvement }\end{array}$ & 61 & $25 \mathrm{GHz}$ & $8 \mathrm{~dB}$ & $\begin{array}{l}\text { A complete system level } \\
\text { prototype, CFBG used as } \\
\text { dispersive medium instead of } \\
\text { DSF }\end{array}$ \\
\hline 4 & [118] & 2012 & $\begin{array}{l}\text { Line increase, stability, insertion } \\
\text { loss, no dispersive medium }\end{array}$ & 53 & $12.5 \mathrm{GHz}$ & $<10 \mathrm{~dB}$ & $\begin{array}{l}\text { Two stage cascaded PMs, } 50 \\
\text { Gb/s PM QPSK transmission } \\
\text { charaterization }\end{array}$ \\
\hline 5 & [119] & 2013 & $\begin{array}{l}\text { Cost and complexity, stability, } \\
\text { insertion loss }\end{array}$ & 21 & $25 \mathrm{GHz}$ & $<3 \mathrm{~dB}$ & $\begin{array}{l}\text { Complexity of [118] reduced } \\
\text { using single high frequency RF } \\
\text { clock. } 160 \mathrm{~Gb} / \mathrm{s} \text { PM QPSK } \\
\text { transmission }\end{array}$ \\
\hline 6 & [120] & 2013 & $\begin{array}{l}\text { Field trial, PM comb enabled } \\
\text { terabit AO-OFDM superchannel, } \\
\text { Flexible transmitter }\end{array}$ & 8 & $25 \mathrm{GHz}$ & $<10 \mathrm{~dB}$ & $\begin{array}{l}\text { Trial 1: } 22 \text { supechannels, } 21.7 \\
\text { Tb/s, } 1503 \mathrm{~km} \text {, } \\
\text { DP-8QAM/DP-QPSK, } \\
\text { Trial2: } 22 \text { superchannels } 16.2 \\
\text { Tb/s, } 2531 \mathrm{~km} \text {, DP-QPSK } \\
\text { modulation }\end{array}$ \\
\hline 7 & [121] & 2014 & $\begin{array}{l}\text { Field trial, PM Comb enabled } \\
\text { terabit Nyquist superchannel } \\
\text { flexible transmitter }\end{array}$ & 8 & $25 \mathrm{GHz}$ & $<10 \mathrm{~dB}$ & $\begin{array}{l}\text { Trial 1: } 41 \text { Nyquist } \\
\text { supechannels, } 54.2 \mathrm{~Tb} / \mathrm{s}, 634 \\
\mathrm{~km} \text {, DP-16QAM, } \\
\text { Trial2: } 41 \text { Nyquist superchannels } \\
\text { 40.5 Tb/s, } 1822 \mathrm{~km} \text {, DP-8QAM } \\
\text { modulation }\end{array}$ \\
\hline
\end{tabular}

(FWS). An integrated design shown in the Fig. 15 (d) was also proposed for scalability and cost efficiency [125]. Although the solution uses multiple frequency shifters, it provides some distinguishing features, not available in other solutions. The FWS is different from other solutions as it:

i) does not require any filtering/de-multiplexing of subcarriers for data modulation (filterless)

ii) can provide frequency locked carriers with both equal (symmetric) and unequal (asymmetric) frequency spacing

iii) provides improved control of the number of carriers: any line can be turned on/off by turning off the driving RF signal.

However, the scalability of the solution is limited and can only be useful in modules that require 4 to 7 carriers.

5) Polarization modulator (PolM) based optical frequency combs: A PolM is a special PM that can support both TE and TM modes, however with opposite phase modulation indices [134]. A PolM introduces more controllable parameters compared with conventional intensity modulators. The generic schematic for generation of OFCs using PolM is shown in the Fig. 16 (a) [135]. When a linearly polarized incident lightwave, oriented with an angle of $45^{\circ}$ to one principle axis of the PolM, is fed to a modulator, a pair of two complementary phase modulated signals is generated along the two principle axis [136]. In the experiment, a polarization controller, at the input of the PolM, is used for the $45^{\circ}$ alignment. The PolM is driven by a frequency and amplitude tunable RF signal.
The normalized optical field, for the complementary phase modulated signals at the output of the PolM, can be expressed as [135]:

$$
\left[\begin{array}{c}
E_{x 0}(t) \\
E_{y 0}(t)
\end{array}\right] \propto \frac{\sqrt{ } 2 /}{2}\left[\begin{array}{c}
\exp \left(j \omega_{c} t+j \beta \cos \omega_{m} t+j \varnothing\right) \\
\exp \left(j \omega_{c} t-j \beta \cos \omega_{m} t\right)
\end{array}\right]
$$

where $\omega_{c}$ and $\omega_{m}$ are angular frequencies of the lightwave and modulating RF signals respectively. $\beta$ is the phase modulation index given by $\left(\pi V_{m} / V_{\pi}\right)$, where $V_{m}$ is the amplitude of modulating RF signal and $V_{\pi}$ is the half wave voltage of the modulator, $\phi$ is the static phase difference between $E_{x}$ and $E_{y}$ [135]. When the polarization modulated signal is passed through a polarizer, after adjusting the polarization state with the principle axis of the polarizer, polarization-modulation to intensity modulation takes place. The optical field after the polarizer is expressed as [137]:

$$
E_{0} \propto\left[\exp \left(j \beta \sin \omega_{m} t\right) \cos \alpha+\exp \left(j \varnothing-j \beta \sin \omega_{m} t\right) \sin \alpha\right]
$$

where $\alpha$ is the angle between one principal axis of the PolM and the principal axis of the polarizer. Both $\phi$ and $\alpha$ can be adjusted by placing a PC before the polarizer. The nonlinearity of PolM causes the generation of harmonics that make up the tones of the OFC. The optical field of each line can be found using [137]: 

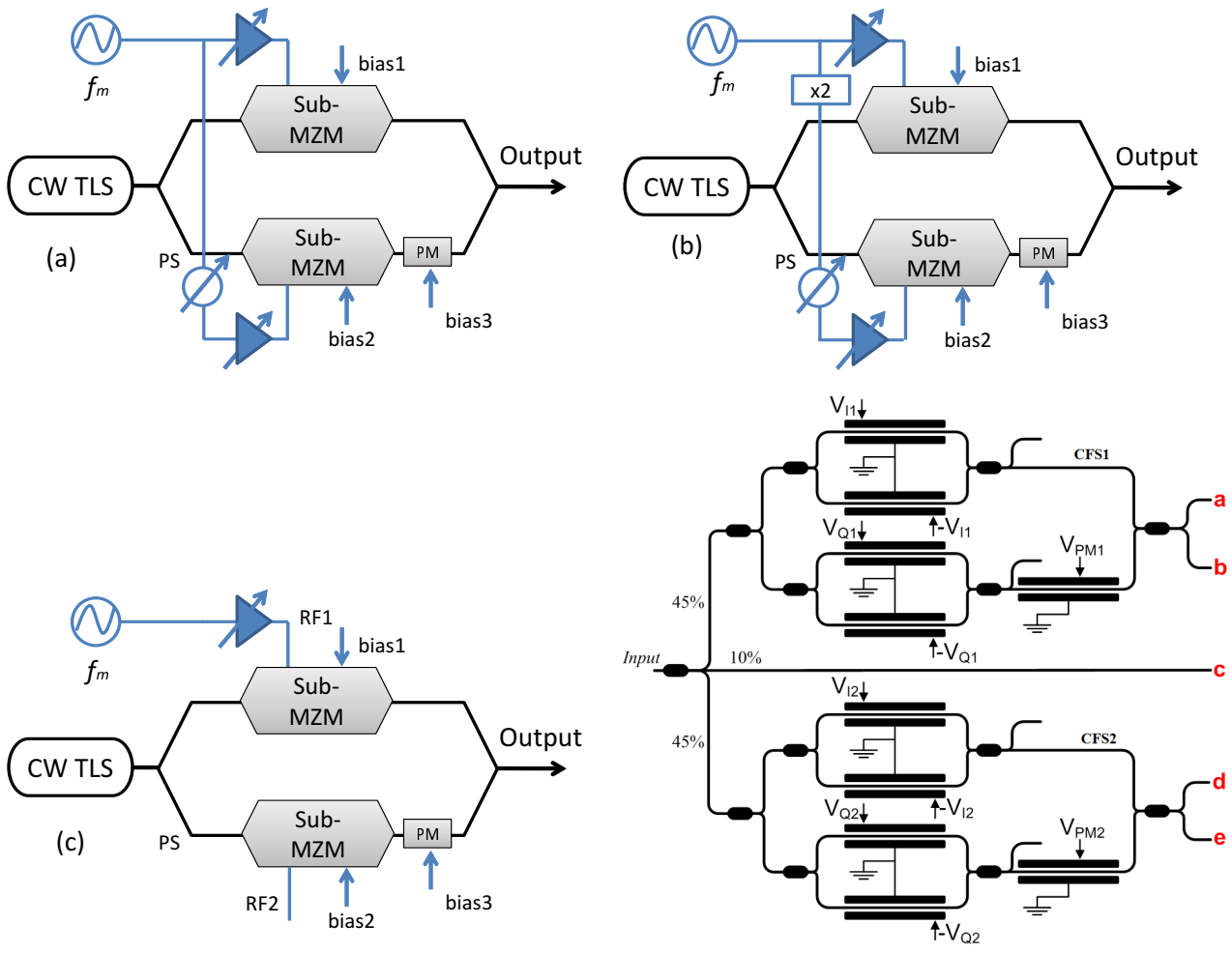

Figure 15: Combs based on nested MZM a) Generic OFC scheme using nested MZM [122] (b) Modified scheme for higher side mode suppression ratio [123] (c) Reduced complexity design [124] (d) A programmable few wavelength source [125].

$$
E_{0} \propto \sum_{k=-\infty}^{+\infty} J_{k}(\beta)\left[\cos \alpha+(-1)^{k} \sin \alpha \exp (j \varnothing)\right] \times \exp \left[j k \omega_{m} t\right]
$$

where $J_{k}(\beta)$ is the $k$ th order of the Bessel function of the first kind.

The combination of a PolM and a polarizer is equivalent to an intensity modulator, but with an additional third parameter to be controlled. In the conventional intensity modulators, $\alpha$ is fixed due to $50 / 50$ splitting ratio and only $\varphi$ and $\beta$ can be tuned. The combination of PolM and polarizer provides the tuning of $\alpha$, thus increasing the possibility of increasing the number of lines and enhancing the spectral flatness. Healy et al. [138] proposed and demonstrated the first OFC using two PolM in cascade, configured as amplitude modulators for CoWDM systems, as shown in the Fig. 16 (b). The output comb provided eleven lines spaced at $42.6 \mathrm{GHz}$ with $<2 \mathrm{~dB}$ spectral flatness. Chao $\mathrm{He}$ et al. [137] proposed a similar scheme, here the output OFC had $<1 \mathrm{~dB}$ spectral flatness and was able to provide 5 lines spaced at $17.5 \mathrm{GHz}, 20 \mathrm{GHz}$ and 25 $\mathrm{GHz}$ as well as 25 lines spaced at $3.5 \mathrm{GHz}, 4 \mathrm{GHz}$ and $5 \mathrm{GHz}$. The PolM based OFCs do not require DC bias voltages and as a result do not suffer from bias drift issues. This in turn ensures stable operation of the OFC. However, the solution requires multiple polarization controllers and generates fewer comb lines for larger frequency spacing. The larger FSRs are very important for high capacity transmission. Authors in [139] proposed an OFC based on cascaded polarization and phase modulators, as shown in Fig. 16 (c). The solution avoided the use of multiple RF sources [137] and frequency dividers or multipliers. The OFC provided 13, 11 and 9 lines, with amplitude variations of $2.1 \mathrm{~dB}, 1.3 \mathrm{~dB}$ and $1 \mathrm{~dB}$ respectively, for a FSR of $10 \mathrm{GHz}$. The theoretical analysis proved that the scheme can also provide 15, 17 and 19 lines, if high RF powers are applied.

Chen et al. [135] managed to increase the number of comb lines from 5 to 7 and achieve a spectral flatness of $1.8 \mathrm{~dB}$, by using a single PolM, driven with a single RF source and a flatness improvement block. The idea behind the flatness improvement is to separate the odd and even lines at the output of the polarizer (a polarization beam splitter) by tuning the $\alpha$ and $\phi$ parameters using a polarization controller. The even lines are then amplified and recombined with odd lines using a polarization beam combiner (PBC). Alternatively, odd lines can be attenuated and combined with the even lines, thus avoiding the use of an amplifier, whilst achieving improved flatness. This technique was used to generate an OFC with FSRs of 3, 5, 7.5 and $10 \mathrm{GHz}$. Efforts in [140], [141] introduced the use of optoelectronic oscillators (OEO), replacing conventional RF sources, as shown in the Fig. 16 (e). Wang and Yao [140] proposed an OFC similar to [137], but using two PolMs. The OEO was implemented using the first PolM, phase shifted fiber Bragg grating (PS-FBG) and a photo-detector (PD). Secondly, both PolMs were driven by the signal from the OEO. The wavelength tuning was achieved by employing two tunable lasers (TLSs). The two PolMs functioned as a cascaded MZM and a PM. The generated comb consisted of 9 or 15 lines that could be tuned from 
Table VII: OPTICAL FREQUENCY COMBS BASED ON NESTED MZM

\begin{tabular}{|c|c|c|c|c|c|c|c|}
\hline $\begin{array}{l}\text { Sr. } \\
\text { No. }\end{array}$ & Ref. & Year & Target/ Issue Addressed & No. of Lines & $\begin{array}{l}\text { Carrier } \\
\text { Spacing }\end{array}$ & Flatness & $\begin{array}{l}\text { Application/ Technique } \\
\text { improvement }\end{array}$ \\
\hline 1 & {$[122]$} & 2007 & $\begin{array}{l}\text { First comb using Nested MZM, } \\
\text { Flatness }\end{array}$ & 9 & $10 \mathrm{GHZ}$ & $<2 \mathrm{~dB}$ & Experimental comb \\
\hline 2 & [126] & 2011 & Number of lines, Carrier spacing & $\begin{array}{l}\text { variable } 2, \\
3,4,7\end{array}$ & $\begin{array}{l}10-60 \mathrm{GHz} \\
(2 \text { lines })\end{array}$ & $\begin{array}{l}\sim 1 \mathrm{~dB} \text { or } \\
\text { below }\end{array}$ & $\begin{array}{l}\text { Detailed simulations based } \\
\text { analysis and experimental } \\
\text { flexible comb demonstration }\end{array}$ \\
\hline 3 & [123] & 2014 & $\begin{array}{l}\text { SMSR improvement, Number of } \\
\text { lines }\end{array}$ & $\begin{array}{l}5,7,9 \text { for } \\
10 \mathrm{GHZ} \\
3 @ 20 \mathrm{GHz} \\
\text { and } 2 @ 40 \\
\mathrm{GHz}\end{array}$ & $\begin{array}{l}\text { Variable } \\
10-40 \mathrm{GHz}\end{array}$ & $\begin{array}{l}<3 \mathrm{~dB} \text { for } \\
9 \text { lines and } \\
<1.5 \mathrm{~dB} \\
\text { for rest }\end{array}$ & $\begin{array}{l}\text { Experimental comb, SMSR > } 15 \\
\mathrm{~dB} \text {, Also produces Gaussian } \\
\text { shaped comb }\end{array}$ \\
\hline 4 & [127] & 2014 & $\begin{array}{l}\text { Nyquist pulse generation using } \\
\text { comb based on IQ }\end{array}$ & 5 & $\begin{array}{l}\text { variable } 10, \\
20,30 \mathrm{GHz}\end{array}$ & $1 \mathrm{~dB}$ & $\begin{array}{l}\text { Single arm of modulator fed } \\
\text { with RF, SMSR }>19 \mathrm{~dB}\end{array}$ \\
\hline 5 & [124] & 2014 & $\begin{array}{l}\text { Reduced Complexity } \\
\text { Reduces complexity by using } \\
\text { only one RF port of IQ. SMSR } \\
29 \mathrm{~dB} \text { for } 5 \text { lines }\end{array}$ & 5,7 & $25 \mathrm{GHz}$ & $<1 \mathrm{~dB}$ & $\begin{array}{l}\text { Experimental comb. No multiple } \\
\text { synchronized RF } \\
\text { sources/frequency multipliers, no } \\
\text { phase shifters, no RF power } \\
\text { balancing /adjustment }\end{array}$ \\
\hline 6 & [39] & 2014 & $\begin{array}{l}\text { Comb enabled SDN controlled } \\
\text { SBVT, SBVT slice-ability }\end{array}$ & 3,5 & $\begin{array}{l}6.25-37.5 \\
\mathrm{GHz}\end{array}$ & $\sim 1 \mathrm{~dB}$ & $\begin{array}{l}\text { First SDN controlled SBVT } \\
\text { demonstration along with } \\
\text { slice-ability feature }\end{array}$ \\
\hline 7 & {$[125]$} & 2016 & $\begin{array}{l}\text { Control on number of lines, } \\
\text { Asymmetric spacing, filter-less } \\
\text { source, Scalability }\end{array}$ & 3,5 & $\begin{array}{l}6.25-37.5 \\
\mathrm{GHz}\end{array}$ & $\sim 1 \mathrm{~dB}$ & $\begin{array}{l}\text { Experimental comb, asymmetric } \\
\text { carrier spacing and slice-ability } \\
\text { demonstration, } 420 \mathrm{~Gb} / \mathrm{s} \\
\text { Transmission. Integrated design } \\
\text { proposed for scalability }\end{array}$ \\
\hline
\end{tabular}

$1500 \mathrm{~nm}$ to $1580 \mathrm{~nm}$ and achieved a tunable FSR ranging from 6.6 to $15.3 \mathrm{GHz}$. The frequency spacing was tuned by controlling the interval between wavelength of TLS and notch of the PS-FBG. Although in this case, the OEO replaces the RF source, the laser and Bragg grating notch stability is crucial to the performance of the comb. Moreover, two input lasers are needed to achieve both FSR and central wavelength tuning. Recently, an interesting comb design based on single PolM [141] that included an OEO and Brillouinassisted power equalizer (BAPE) for better spectral flatness, was reported. The idea is to initially create two OFCs, having different power levels at the output of the PolM and then feed them in to the BAPE. The BAPE flattens the OFC by attenuating the optical lines having power beyond the threshold of stimulated Brillouin scattering (SBS) and amplifying the lines having power below the threshold. This technique is employed to generate an OFC with 5, 7, 9 and 11 lines that exhibit a spectral flatness of $0.5,1.5,2$ and $4.1 \mathrm{~dB}$ respectively, at a FSR of $10 \mathrm{GHz}$. These are the highest number of OFC lines reported so far using a single PolM. In [142], the authors proposed a complementary frequency shifter (CFS) based on a PolM, as depicted in Fig. 16 (f). The CFS is placed in single and dual re-circulating frequency shifting (RFS) loop to produce 100 optical lines (Fig. 16 (h)). However, the results are based on theoretical analysis and simulations using 'VPItransmissionMaker'. A PolM based CFS has several advantages over conventional CFS based on IQ modulators. It requires fewer phase modulators, no dc bias controller, provides higher bandwidth and has low RF power requirement. Table VIII summarizes the works related to PolM based combs.
6) Ultra-broad parametric combs based on nonlinear spectral broadening: OFCs, based on phase modulation in cascaded electro-optic modulators, can provide good flatness and flexibility, but their overall the spectral bandwidth is limited. The spectral width of the combs can be increased either by using cavity based RFS loops, or by nonlinear spectral broadening. Although combs employing RFS loops provide a large number of lines, the cavities must be stabilized to support frequency pitch or carrier spacing tuning. The OFCs using nonlinear spectral broadening provide an alternative, cavityless approach to generating an ultra-broad output spectrum. This can be achieved via the nonlinear compression of high repetition rate optical pulses (provided by conventional EO combs) in highly nonlinear passive medium such as highly nonlinear fiber (HNLF). In the time domain, an OFC corresponds to a pulse train, which can be expressed in general by

$$
g(t)=A(t) e^{j \varphi(t)}
$$

where, $A(t)$ is amplitude and $\phi(t)$ is phase of the pulse. The Fourier transform of the pulse train represents the spectrum of a band-limited OFC and can be expressed as [143]:

$$
\sum_{k=-\infty}^{\infty} G(f) . \delta(f-k / \Delta T)=\digamma \sum_{n=-\infty}^{\infty} g(t) * \delta(t-n \Delta T)
$$

where, $\digamma$ denotes the Fourier transform, $\delta\left(t-t_{0}\right)$ is Dirac delta function centered at $t_{0}, \Delta T$ is the pulse repetition rate and $G(f)$ is the Fourier transform of the pulse waveform [143]. Therefore, any periodic temporal pulsed emission can also be 

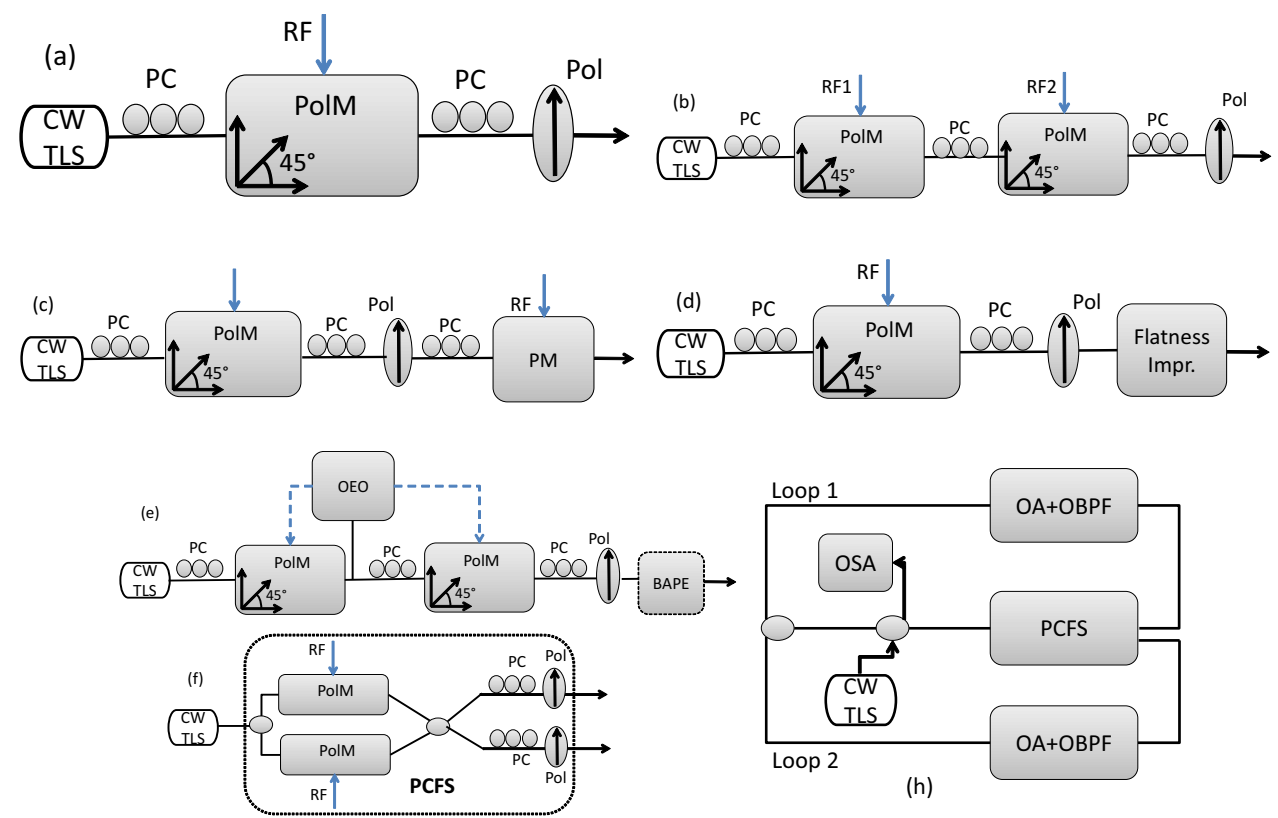

Figure 16: Combs based on polarization modulators (a) Generic OFC scheme using PolM (b) Comb using cascaded PolM [138] (c) OFC scheme based on cascaded PolM and PM [139] (d) Single PolM based comb with additional flatness improvement block [135] (e) PolM based combs with optoelectronic oscillator (OEO) [140], [141] (f, h) Complementary frequency shifter using PolMs and its used in RFS loop [142].

seen as a spectral comb [144]. The comb spectral envelope is defined by the Fourier transform of the pulse shape, $G(f)$, and the comb pitch i.e. the carrier spacing, is proportional to the pulse repetition rate $\Delta T$. Therefore, a wide frequency comb can be generated by either reducing the temporal width of the pulse, $A(t)$, by applying a strong phase modulation to high intensity optical pulses, or a combination of both. The high intensity pulses modulate the refractive index of the nonlinear material (i.e., called the mixer) and cause a strong phase modulation proportional to the intensity profile. This results in the generation of a wide frequency comb. Such nonlinear interaction is also referred to as the parametric process, because no change in material energy levels occurs during the process [144]. The efficient generation of the new frequency tones is dependent on the simultaneous fulfillment of two conditions:

1) high aggregate nonlinear figure of merit (FoM)

2) wide-band phase matching.

The FoM of a nonlinear mixer is defined as the product of optical power $(P)$, effective interaction length $(L)$ and mixer nonlinearity $(\gamma)$ [145]. Wide-band phase matching is essential to maintain a high $F o M$, because not only are the optical pulses with high peak power required, but also the peak power of the pulses should remain high, while propagating inside the nonlinear material. For example, material dispersion can cause early pulse destruction leading to a significant reduction of the FoM [144].

In traditional nonlinear mixers, pulsed MLL were widely used as high peak power pulsed sources (peak powers in excess of $1 \mathrm{~kW}$ ) [146]. However, the ability to change the comb frequency pitch is effectively lost in MLL, since the cavity cannot be simultaneously stabilized and re-configured
[147]. Moreover, if the pulse seed is based on the cavity (MLL), such a comb generation solution cannot be treated as a truly cavity-less. If $\mathrm{CW}$ lasers are to be used as the seed, replacing MLLs, a longer length of the nonlinear mixer would be required to achieve the same phase shift $(\gamma \operatorname{Pmax} L)$ or FoM. For instance, if two CWs injecting $1 W$ of power are used, several kilometers of $\operatorname{HNLF}\left(\gamma \sim 20 \mathrm{~W}^{-1} \mathrm{Km}^{-1}\right)$ would be required to generate a comb with hundreds of lines. This would make the solution less compact and less practical. The increased size of a mixer would also make controlling the dispersion and suppressing the Brillouin effects difficult. In order to achieve high a FoM using low power $\mathrm{CW}$ seeds, a high peak power waveform must be synthesized. Authors in [148], [149] proposed multi frequency seeds by modulating a single CW laser with electro-optic modulator, driven by an electrical tone, shown in the Fig. 17 (a). Myslivets et al. introduced two $\mathrm{CW}$ tones as a seed that can be easily tuned to produce an arbitrary comb frequency pitch and demonstrated a new mixer architecture based on 'chirp-compress-and-mix cycle' for wideband comb generation as depicted in Fig. 17 (b) [145]. The new architecture relies on the efficient creation of higherorder mixing tones in phase-matched nonlinear fiber stages, separated by a linear compressor. Wideband generation was enabled by precise dispersion engineering of multiple-stage parametric mixers. The beating of two input $\mathrm{CW}$ tones in the first nonlinear stage $\left(N L_{1}\right)$, causes positive chirp induced by Kerr effect and produces a limited number of higher order frequency tones. The chirped signal from $N L_{l}$ is then passed through a SMF, having negative second-order dispersion $\left(\beta_{2}\right)$ and acting as a compressor. The compressor stage compensates the chirp and provides high power, narrow width pulses in the time domain. However, the compression process is not ideal 
Table VIII: OPTICAL FREQUENCY COMBS BASED ON POLARIZATION MODULATORS

\begin{tabular}{|c|c|c|c|c|c|c|c|}
\hline Sr. No. & Ref. & Year & Target/ Issue Addressed & $\begin{array}{l}\text { No. of } \\
\text { Lines }\end{array}$ & $\begin{array}{l}\text { Carrier } \\
\text { Spacing }\end{array}$ & Flatness & $\begin{array}{l}\text { Demonstration/ } \\
\text { Technique improvement }\end{array}$ \\
\hline 1 & [138] & 2007 & $\begin{array}{l}\text { First comb using two } \\
\text { polarization modulators, Avoid } \\
\text { use of bias controllers }\end{array}$ & 11 & $42.6 \mathrm{GHz}$ & $<2 \mathrm{~dB}$ & $\begin{array}{l}\text { Comb generation for CoWDM } \\
\text { system. SMSR }>12 \mathrm{~dB}\end{array}$ \\
\hline 2 & [137] & 2012 & $\begin{array}{l}\text { Tunable comb source using } \\
\text { cascaded PolM, Flatness, } \\
\text { Number of lines }\end{array}$ & $\begin{array}{l}5 \text { and } \\
25\end{array}$ & $\begin{array}{l}17.5-25 \mathrm{GHz} \\
5 \text { lines, } \\
3.5-5 \mathrm{GHz} \\
25 \text { lines }\end{array}$ & $\begin{array}{l}0.49 \mathrm{~dB} \text { for } \\
25 \mathrm{GHz}\end{array}$ & $\begin{array}{l}\text { Experimental comb, theoretical } \\
\text { analysis, no transmission } \\
\text { characterization }\end{array}$ \\
\hline 3 & [139] & 2013 & $\begin{array}{l}\text { PolM and PM cascaded, } \\
\text { equivalent of cascaded IM and } \\
\text { PM, stability }\end{array}$ & $\begin{array}{l}13,11, \\
9\end{array}$ & $10 \mathrm{GHz}$ & $\begin{array}{l}0.94 \mathrm{~dB} \text { for } \\
5 \mathrm{GHz}\end{array}$ & $\begin{array}{l}\text { Experimental comb, single RF } \\
\text { source }\end{array}$ \\
\hline 4 & [135] & 2013 & $\begin{array}{l}\text { Flatness, Line increase } \\
\text { Single PolM based comb }\end{array}$ & 7 & $\begin{array}{l}3,5,7.5,10 \\
\mathrm{GHz}\end{array}$ & $\begin{array}{l}2.1,1.3,1 \\
\mathrm{~dB}\end{array}$ & Experimental comb \\
\hline 5 & [140] & 2013 & $\begin{array}{l}\text { Photonic RF synthesis, Tunable } \\
\text { comb based on cascaded PolMs }\end{array}$ & $\begin{array}{l}9 \text { for } \\
8-12 \\
\mathrm{GHz}, 15 \\
\text { for } 8 \\
\mathrm{GHz}\end{array}$ & $6.6-15 \mathrm{GHz}$ & $1.8 \mathrm{~dB}$ & $\begin{array}{l}\text { Comb alone, carrier spacing and } \\
\text { central wavelength tuning } \\
\text { demonstrated }\end{array}$ \\
\hline 6 & [142] & 2014 & $\begin{array}{l}\text { Number of lines, stability, } \\
\text { carrier spacing, CFS based on } \\
\text { PolM proposed and used in } \\
\text { single and double RFS loops }\end{array}$ & 100 & $>40 \mathrm{GHz}$ & $\begin{array}{l}1.5 \mathrm{~dB}, 2.2 \\
\mathrm{~dB}\end{array}$ & $\begin{array}{l}\text { Simulations only, Fewer Phase } \\
\text { modulators than other RFS } \\
\text { schemes, Higher FSR than } \\
\text { Conventional CFS }\end{array}$ \\
\hline 7 & {$[141]$} & 2014 & Flatness, photonic RF synthesis & $\begin{array}{l}5,7,9 \\
11\end{array}$ & $10 \mathrm{GHz}$ & $\begin{array}{l}0.5 \mathrm{~dB} \\
1.5 \mathrm{~dB} \\
2 \mathrm{~dB} \\
4.1 \mathrm{~dB}\end{array}$ & $\begin{array}{l}\text { Brillouin assisted power } \\
\text { equalizer to improve the flatness }\end{array}$ \\
\hline
\end{tabular}

and its imperfection leading to parasitic side-lobes, causing interference. The side-lobes prevent the generation of a flat top intensity pulse, which results in spectral ripple (poor flatness) [86], [145]. The output of the SMF is then passed through the second nonlinear mixer stage $\left(N L_{2}\right)$, where four photon mixing interaction produces a wideband OFC. The chirp-compressmix cycle improves the achievable comb bandwidth, which can be further increased by adding more stages. Although the scheme decouples pulse generation from cavity imposed limits, the output spectrum of this cavityless scheme exhibits poor spectral flatness (less than $10 \mathrm{~dB}$ ). Parametric combs inherently suffer from spectral linewidth growth problem that scales with the four wave mixing (FWM) tone order. This effect reduces the quality of higher-order tones and contributes to the degradation of OSNR of the OFC lines. Tong et al. demonstrated an OFC spanning $200 \mathrm{~nm}$, providing more than hundred lines, while preserving the linewidth. Linewidth preservation was obtained by seeding the comb with phase correlated CW pumps. Phase synchronization between pumps was achieved by injection-locking two distinct slave lasers with two phase-modulated sidebands of a single LO. The injection locking of the slave lasers, in addition to preserving linewidth, also regenerates (amplifies) the master tones and reduces the relative intensity noise (RIN) [152].

As explained in earlier sections, when the pulses are accompanied by a strong quadratic phase modulation, the magnified profile of the pulses' intensity is mapped onto the comb spectrum i.e., flat-top combs can be generated by quadratic phase modulation of flat top pulses. However, the spectral bandwidth of such combs is limited. On the other hand, parametric combs provide a wide bandwidth, but have poor flatness. The authors in [153] used the combination of both methods to generate a wide-band flat-topped OFC. An OFC consisting of 365 spectral lines at $10 \mathrm{GHz}$ spacing, with 3.5 $\mathrm{dB}$ spectral flatness and an overall spectral bandwidth of 28 $\mathrm{nm}$, was presented using a seed comb with Gaussian envelope and HNLF as a NL mixer. Atai et al. improved the flatness by acting on the pulse shape, carving it precisely and introducing the regeneration stage to suppress the side-lobes (see Fig. 17 (c)) [147].

For high quality comb generation it is essential to synthesize the high peak power waveforms prior to the frequency generation process. The periodic beat tones can be generated by the AM, followed by the PM, to provide the chirp, prior to subsequent dispersion-mediated temporal compression in standard SMF. The compression of high peak power pulses, propagating in the normal dispersion regime, leads to shockwave or optical wave breaking [154]. Different frequency components generated by SPM meet and interfere at the same location [10]. As a result of the shock-wave phenomenon, the peak intensity increases by more than two orders of magnitude. This translates to a hundred-fold increase in the FoM of the HNLF mixer [147]. However, for flat comb generation, reshaping of the seed pulse, after the compression stage, is essential to suppress the side modes. This is done in order to keep the high power and linear chirp part of the pulse, whilst removing the nonlinearly chirped side-lobes [145]. A nonlinear optical loop mirror (NOLM) transfer function can provide such properties. Authors in [68], [147] proposed a regeneration stage based on the NOLM to suppress the sidelobes. In the experiments, the output of the NOLM-based regeneration stage provided near-ideal seed properties for the 

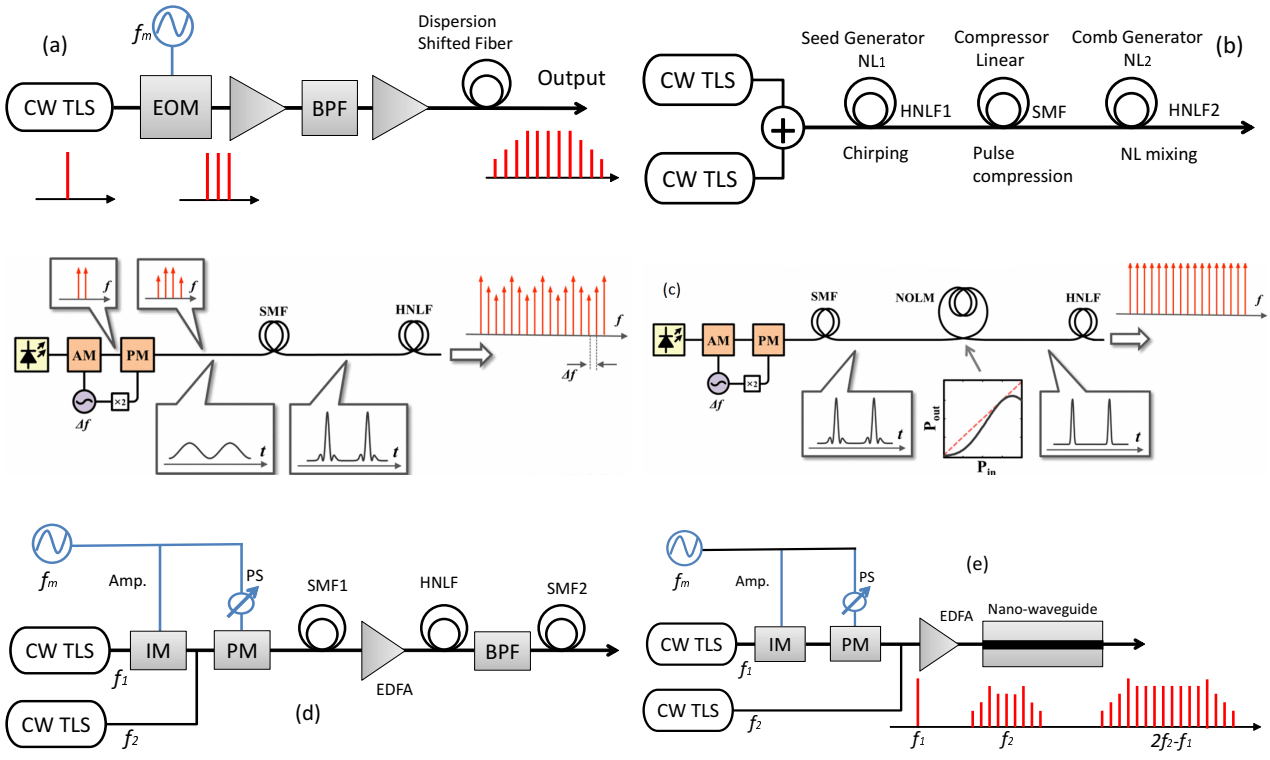

Figure 17: Parametric combs (a) Parametric comb with multi-frequency seed [148], [149] (b) CW seeded chirp-compress-mix cycle [145] (c) CW-seeded Shock-Wave Mixer Principle: (left) phase modulator (PM) generates chirp to induce shock-wave (compression) and increase peak power prior to the parametric comb generation in HNLF; imperfect pulse compression leads to pulse sidelobes, resulting in comb spectral ripple; (right) NOLM transfer function is used to regenerate compressed waveform and seed mixing stage (HNLF) with near-ideal seeds, eliminating comb ripple. [147] (d) Comb based on cascaded FWM [150] (e) Integrated parametric comb using FWM in an on-chip nano-waveguide [151].

next mixing stage (HNLF), resulting in a highly flat wideband frequency comb. A $120 \mathrm{~nm}$ wide OFC with less than $2 \mathrm{~dB}$ spectral flatness, providing 1500 lines spaced at $10 \mathrm{GHz}$ and having $40 \mathrm{~dB}$ OSNR, was successfully demonstrated. Subsequently, the same group reported first use of such parametric comb as a coherent optical carrier in a QPSK transmitter. The performance of the comb based transmitter exceeded that of the standard tunable coherent device, while providing lower frequency uncertainty and drift [68]. In continuation to previous work on spectral flatness improvement through re-shaping, in addition to NOLM, Ataie et al. introduced the use of nonlinear optical amplifying loop mirror (NALM) in the regeneration stage [143]. The difference between the two can be understood by looking into the mathematical representations of transfer function for NOLM and NALM. The NOLM power transfer function can be expressed as [143]:

$$
T=(1-2 \alpha(1-\alpha))(1+\cos (\gamma P L(1-2 \alpha)))
$$

where, $T$ is the NOLM transmittance, $(\alpha)$ is the directional coupler split ratio, $\gamma$ is the nonlinear coefficient, $P$ is the optical power, and $L$ is the nonlinear interaction length. The magnitude of the spectral flatness is proportional to the peakto-pedestal ER. The higher splitting ratio $(\alpha)$ in the NOLM leads to a higher side-lobe suppression. However, as $(\alpha)$ approaches the 0.5 value, the required nonlinearity-length $(\gamma L)$ product, necessary for a NOLM to operate at transparency diverges, making such design impractical. The NOLM uses unequal splitting ratios of a coupler to create the imbalances of nonlinear phase shifts of counter propagating waves in the loop. The NALM is a slightly modified version of the NOLM, with a short bidirectional amplifier in the loop and can work with a 50:50 coupler to further enhance the ER. NALM uses the amplifier to create imbalance in the nonlinear phase shifts of counter propagating waves in the loop, thus providing a higher ER, simultaneously compensating for the losses (amplifying the waveforms). However the amplifier introduces additional noise. The transfer function of the NALM is given by [143]:

$$
T=\frac{1}{2} G A\left(1-\cos \left(\frac{1}{2} \gamma P L(G-A)\right)\right)
$$

where, $T$ is the NALM transmittance, $G$ and $A$ are the gain and attenuation within the loop respectively, $\gamma$ is the nonlinear coefficient, $P$ is the optical power. $L$ is the nonlinear interaction length. In principle, multiple NOLMs/NALMs can be cascaded to increase the spectral flatness of the comb. Two different combs with coarse $(100 \mathrm{GHz})$ and dense $(10$ $\mathrm{GHz}$ ) tone spacing, exhibiting a $4 \mathrm{~dB}$ flatness over $100 \mathrm{~nm}$ and sub- $2 \mathrm{~dB}$ spectral flatness over $120 \mathrm{~nm}$ bandwidth (1500 lines), were produced respectively [143]. Subsequently, a comb based 20 Gbaud 16QAM transmitter was demonstrated using carriers from an OFC (based on the principle described above) providing 126 lines with a FSR of $100 \mathrm{GHz}$. Each of the OFC line portrayed an OSNR of $\geq 45 \mathrm{~dB}$ and average channel power per line of $7 \mathrm{dBm}$ [155]. The suitability of parametric combs for superchannel and flex-grid transmitters was presented, by demonstrating dynamic re-configuration of comb carrier spacing $(6.25 \mathrm{GHz}$ to $100 \mathrm{GHz})$ for a 16QAM transmitter, with less than $0.5 \mathrm{~dB}$ Q-factor penalty [156]. An ultra-dense and wideband parametric comb, further increasing the number of lines to 1800 , with $6.25 \mathrm{GHz}$ spacing and $3 \mathrm{~dB}$ spectral 
flatness over $100 \mathrm{~nm}(\mathrm{C}+\mathrm{L}$ band), was also demonstrated [144].

Both SPM and FWM can be used for spectral broadening of OFCs in a nonlinear passive medium. The authors in [157] provided a comparative analysis of five different OFC generation schemes, using either SPM or cascaded four wave mixing (CFWM) or both, in HNLF. The experimental results show that when only CFWM occurs in the HNLF, a broadband OFC can be generated, but the spectral flatness is poor. When only SPM takes place in the HNLF, a $10 \mathrm{GHz}$ spaced OFC of $103 \mathrm{comb}$ lines within $5 \mathrm{~dB}$ power deviation is obtained. Whereas, when both CFWM and SPM simultaneously occur in the HNLF, an OFC of 143 comb lines with $4.5 \mathrm{~dB}$ spectral flatness is generated. The authors also investigated how the performance of the OFC is affected by parameters like launched power into the HNLF, central wavelength of seed laser and frequency of RF signal driving modulator.

Supradeepa et al. demonstrated bandwidth scaling and spectral flatness enhancement of an OFC based on intensity and phase modulated CW lasers using CFWM in HNLF, as shown in the Fig. 17 (d). An OFC having 100 spectral lines separated by $10 \mathrm{GHz}$ within a $10 \mathrm{~dB}$ was reported, in which a record 75 lines had $1 \mathrm{~dB}$ amplitude variation [150]. The experiments showed that CFWM process in HNLF increases the bandwidth of the input comb by a factor of five. A comb bandwidth scaling, using FWM in an on-chip silicon nanowaveguide, rather than in HNLF, was demonstrated in [151] and is depicted in Fig. 17 (e). The dispersion characteristics of silicon waveguide were tailored by engineering the waveguide geometry to increase the conversion bandwidth. The FWM process in the waveguide doubled the bandwidth of the seeded comb (generated by the modulation of a CW laser), producing an output comb with more than 100 lines, spaced by $10 \mathrm{GHz}$ and with $5 \mathrm{~dB}$ spectral flatness. This work is a significant achievement for integrated parametric combs, paving the way for their use in future networks, by reducing cost, footprint and power consumption. Table IX presents the summary of reported works.

7) Coupled cavity combs: Resonant schemes are an alternative approach to increase the comb spectral bandwidth. FabryPerot (FP) modulator and fiber ring cavities containing EO modulators are examples of this approach. A FP modulator essentially consists of an EO phase modulator, placed in a FP cavity and driven at a frequency corresponding to the cavity length [159], [160], [161]. An OFC based on FP modulators have been used in frequency metrology [159], [162] and optical pulse train generation [163]. However, they are not suitable for optical communications due to the poor frequency resolution (few $\mathrm{GHz}$ ). To achieve a fine resolution, the cavity length has to be adjusted precisely, according to operational frequency. This increases the complexity and switching time. Since fiber rings have long cavities, a much narrower $(\mathrm{MHz})$ frequency spacing can be achieved. This however, makes stable operation very difficult, unless multiple cavity modes per comb line are permitted, to stabilize the comb [164]. Fiber ring based comb generators are realized by repetitive frequency shifting of an optical signal circulating in the loop. A generic scheme of fiber ring comb generators is shown in the
Fig. 18 (a). A light signal from a narrow linewidth $\mathrm{CW}$ source is supplied to the loop, which is composed of a 50:50 coupler, an EO modulator, an optical amplifier (OA), a tunable optical band-pass filter (BPF) and a polarization controller. The OA is used to compensate the optical losses in the loop and the BPF is used to set the spectral width of the comb. Such combs can be broadly divided into two major categories, based on frequency shifting process:

i) double side band frequency shifting, and

ii) single sideband frequency shifting.

The former type typically uses phase modulators (PM) in the loop, while the latter uses an IQ modulator. Single sideband RFS combs using PolM [142] and a three branch waveguide interferometer [165] have also been proposed recently. Moreover, fiber ring based RFS combs can also be classified based on RFS loop architecture, optical CW seed and modulating RF drive signal type, as shown in the Fig. 18 (b). In the following we shall review different works related to both RFS comb types.

a) Fiber ring cavities containing PM based dual sideband frequency shifter : In the fiber ring schemes, the optical signal is passed multiple times through a modulator placed in a fiber ring or loop, acting as an external resonator, to increase the OFC bandwidth. Ho and Kahn proposed a fiberring-based optical frequency comb in 1993, similar to generic schemes shown in Fig. 18 (a) [166]. The PM is enhanced by multiple passes of light through the PM driven by a sine wave signal. Up-shifted and down-shifted sidebands from the frequency of the seed CW source are created on each pass, creating a comb-like spectrum. The amplifier in the loop compensates for the round trip loss. The authors also provided a time domain analysis of the scheme. Such an OFC was experimentally presented by Bennett et al. providing 103 lines at a FSR of $25 \mathrm{GHz}$ in a $1.8 \mathrm{THz}$ span and within a 40 $\mathrm{dB}$ power envelop [164]. Later, frequency domain analysis was provided to understand the effects of fiber dispersion, the non-flat gain profiles, and the RF mismatch. OFCs having a $1 \mathrm{THz}$ (100 lines, $6 \mathrm{~dB}$ flatness) and $2 \mathrm{THz}$ (200 lines, $10 \mathrm{~dB}$ flatness) spectral bandwidth were reported in [167], [168]. The authors also demonstrated frequency spacing tuning from $6 \mathrm{GHz}$ to $12.5 \mathrm{GHz}$ in $12.5 \mathrm{MHz}$ steps, while maintaining the spectral profile. The switching time was less than one second. For a fiber ring based OFC, the comb width can be limited by dispersion and other phase-mismatching conditions. The comb width of dispersion limited fiber rings is not affected by the modulation frequency i.e. the width would remain the same and fewer lines will be generated for higher modulating frequencies. The span of the comb can be improved by increasing the modulation depth and reducing the chromatic dispersion in the ring. Loop control circuits are essential for the stable operation [167]. Zhang et al. proposed an OFC containing two cascaded PMs [169] in the loop. Phase modulators have low insertion loss and do not need DC bias control. The number of lines in RFS combs is limited by accumulation of noise from the optical amplifier (i.e. EDFA). An IQ modulator based FS generates one line in each circulation, whereas a higher number of lines (22 in this 
Table IX: ULTRA-BROAD PARAMETRIC COMBS BASED ON NONLINEAR SPECTRAL BROADENING

\begin{tabular}{|c|c|c|c|c|c|c|c|}
\hline $\begin{array}{l}\text { Sr. } \\
\text { No. }\end{array}$ & Ref. & Year & Target/ Issue Addressed & $\begin{array}{l}\text { No. of } \\
\text { Lines }\end{array}$ & $\begin{array}{l}\text { Carrier } \\
\text { Spacing }\end{array}$ & Flatness & $\begin{array}{l}\text { Application/ Technique } \\
\text { improvement }\end{array}$ \\
\hline 1 & [146] & 2002 & $\begin{array}{l}\text { Supercontinuum multicarrier } \\
\text { source for WDM systems }\end{array}$ & 1000,106 & $\begin{array}{l}12.5 \mathrm{GHz} \\
25 \mathrm{GHz}\end{array}$ & $\underset{\mathrm{dB}}{10 \mathrm{~dB}, 5}$ & $\begin{array}{c}\text { Nonlinear fiber pumped by } \\
\text { picosecond optical pulses. } \\
\text { Transmission } 106 \times 10 \mathrm{~Gb} / \mathrm{s}, 640 \\
\mathrm{~km}\end{array}$ \\
\hline 2 & [149] & 2010 & $\begin{array}{l}\text { Carrier spacing tunability, } \\
\text { Replaces MLL with } \\
\text { multi-frequency seed by } \\
\text { modulating CW laser. }\end{array}$ & C-band & $25 \mathrm{GHz}$ & $\sim 10 \mathrm{~dB}$ & $\begin{array}{l}\text { Experimental comb, } \\
\text { octave-spanning SC spectra is } \\
\text { generated by passing pulses } \\
\text { through dispersive fiber and } \\
\text { highly nonlinear Te PCF }\end{array}$ \\
\hline 3 & {$[150]$} & 2012 & $\begin{array}{c}\text { Comb bandwidth and spectral } \\
\text { flatness }\end{array}$ & 100 & $10 \mathrm{GHz}$ & $10 \mathrm{~dB}$ & $\begin{array}{l}\text { Experimental comb based on } \\
\text { cascaded FWM in HNLF }\end{array}$ \\
\hline 4 & [145] & 2012 & $\begin{array}{c}\text { Arbitrary carrier spacing, } \\
\text { Proposed new design of NL } \\
\text { mixer based on } \\
\text { chirp-compress-mix cycle }\end{array}$ & $150 \mathrm{~nm}$ & Variable & $\sim 15 \mathrm{~dB}$ & $\begin{array}{l}\text { Experimental comb, No need for } \\
\text { pulsed laser source }\end{array}$ \\
\hline 5 & [152] & 2012 & $\begin{array}{c}\text { Phase coherence, Line-width } \\
\text { preservation }\end{array}$ & $200 \mathrm{~nm}$ & $\begin{array}{l}200 \mathrm{GHz} \\
\text { and } 400 \\
\mathrm{GHz}\end{array}$ & $12 \mathrm{~dB}$ & $\begin{array}{l}\text { Experimental comb, Line-width } \\
\text { preservation over larger comb } \\
\text { bandwidth using phase } \\
\text { synchronized CW lasers. } 35 \mathrm{~dB} \\
\text { OSNR }\end{array}$ \\
\hline 6 & [147] & 2013 & $\begin{array}{c}\text { Flatness, Number of lines, } \\
\text { OSNR, Shockwave Mixer design } \\
\text { proposed }\end{array}$ & 1500 & $10 \mathrm{GHz}$ & $2 \mathrm{~dB}$ & $\begin{array}{c}\text { Experimental flat comb, } 40 \mathrm{~dB} \\
\text { OSNR }\end{array}$ \\
\hline 7 & [68] & 2013 & $\begin{array}{l}\text { Parametric comb as coherent } \\
\text { optical carrier and LO }\end{array}$ & $\begin{array}{l}120(100 \\
\mathrm{nm})\end{array}$ & $100 \mathrm{GHz}$ & $3-4 \mathrm{~dB}$ & $\begin{array}{l}\text { BTB QPSK transmission, } 5 \\
\mathrm{dBm} \text { power per tone, } 47 \mathrm{~dB} \\
\text { OSNR, } 0.2 \mathrm{~W} \text { power } \\
\text { consumption per tone }\end{array}$ \\
\hline 8 & [157] & 2013 & $\begin{array}{l}\text { Comb quality, compares } \\
\text { different comb generation } \\
\text { schemes based on cascaded } \\
\text { FWM and SPM inn HNLF }\end{array}$ & 103,143 & $10 \mathrm{GHz}$ & $\begin{array}{c}5 \mathrm{~dB}, 4.5 \\
\mathrm{~dB}\end{array}$ & $\begin{array}{l}5 \text { different OFC generation } \\
\text { schemes compared. Investigates } \\
\text { effect of launch power, laser } \\
\text { central wavelength and RF } \\
\text { signal frequency on the comb } \\
\text { performance }\end{array}$ \\
\hline 9 & [143] & 2014 & $\begin{array}{l}\text { Flatness, NL mixer regeneration } \\
\text { stage analysis and design rules }\end{array}$ & $\begin{array}{c}100 \mathrm{~nm} \\
\& 120 \mathrm{~nm} \\
1500\end{array}$ & $\begin{array}{c}100 \mathrm{GHz} \& \\
10 \mathrm{GHz}\end{array}$ & $4 \mathrm{~dB}, 2 \mathrm{~dB}$ & $\begin{array}{l}\text { Theoretical and experimental } \\
\text { investigation of regeneration } \\
\text { stage and its effects on flatness. } \\
\text { Defines spectral equalization } \\
\text { principle. }\end{array}$ \\
\hline 10 & [158] & 2014 & $\begin{array}{l}\text { Analysis of parametric comb for } \\
\text { telecom and datacom } \\
\text { applications }\end{array}$ & N/A & N/A & N/A & $\begin{array}{l}\text { Highlights key qualities and } \\
\text { defines certain parameters for } \\
\text { evaluation }\end{array}$ \\
\hline 11 & {$[155]$} & 2014 & $\begin{array}{c}\text { Comb enabled transmission, } \\
\text { C }+\mathrm{L} \text { band comb }\end{array}$ & 126 & $100 \mathrm{GHz}$ & $\sim 3 \mathrm{~dB}$ & $\begin{array}{c}\text { Comb based } 20 \text { GBaud 16QAM } \\
\text { transmitter, } 5 \text { stage mixer, } 45 \mathrm{~dB} \\
\text { OSNR, average power } 7 \mathrm{dBm}\end{array}$ \\
\hline 12 & [156] & 2014 & $\begin{array}{l}\text { Dynamic reconfiguration } \\
\text { 16QAM flex-grid superchannel } \\
\text { using carrier spacing tunability }\end{array}$ & $70 \mathrm{~nm}$ & $\begin{array}{c}6.25-100 \\
\mathrm{GHz}\end{array}$ & $2-3 \mathrm{~dB}$ & $\begin{array}{c}\text { Carrier spacing tuned from } 6.25 \\
\text { GHz to } 100 \mathrm{GHz} \text { for } 5 \text { and } 10 \\
\text { GBaud 16QAM transmitter. } \\
\text { Phase stability, BER } \\
\text { performance }\end{array}$ \\
\hline 13 & [151] & 2014 & $\begin{array}{l}\text { Integrated comb, on-chip silicon } \\
\text { nano-waveguide as NL medium }\end{array}$ & 100 & $10 \mathrm{GHz}$ & $5 \mathrm{~dB}$ & $\begin{array}{l}\text { Experimental comb using FWM } \\
\text { in an on-chip nano- waveguide. } \\
\text { HNLF replaced with waveguide }\end{array}$ \\
\hline 14 & {$[144]$} & 2015 & $\begin{array}{l}\text { Ultra-high count coherent } \\
\text { NWDM transmission, Line } \\
\text { spacing tunability }\end{array}$ & 1800 & $6.25 \mathrm{GHz}$ & $3 \mathrm{~dB}$ & $\begin{array}{c}\mathrm{C}+\mathrm{L} \text { band, } 100 \mathrm{~nm} \text { comb, } \\
\text { 16/32/64QAM channels making } \\
32 \mathrm{~Tb} / \mathrm{s}\end{array}$ \\
\hline
\end{tabular}

case) can be generated using cascaded PMs in one iteration, thus increasing the comb width and improving worst case TNR. The generated comb had 113 lines at $25 \mathrm{GHz}$ spacing, 5 $\mathrm{dB}$ spectral flatness, $26 \mathrm{~dB}$ TNR in $22.6 \mathrm{~nm}$ and was also used to transmit $11.2 \mathrm{~Tb} / \mathrm{s}$ signals [170]. The phase deviation of RF drive signals on the two phase modulators should be carefully controlled to generate more subcarriers. The same group later demonstrated the generation of a full C-band coherent and frequency-locked multi-carriers, by using two RFS loops based on phase modulator with external injection-seeding. Each loop 

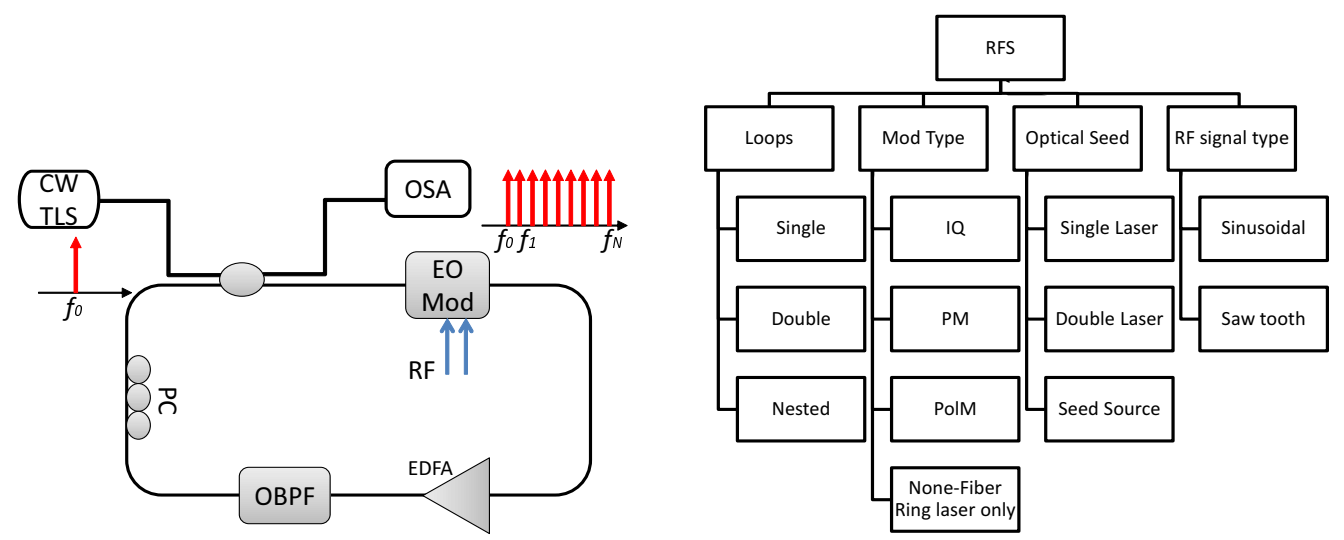

Figure 18: (left) Generic architecture of fiber ring re-circulating frequency shifter comb (right) Classification of reported fiber ring RFS schemes.

has only one phase modulator: one loop is used to generate long wavelength and the other short wavelength subcarriers. A full C-band comb with 178 subcarriers and $26 \mathrm{GHz}$ subcarrier frequency spacing, with $3 \mathrm{~dB}$ spectral flatness and $20 \mathrm{~dB}$ TNR covering nearly $36.66 \mathrm{~nm}$, was generated [171]. The authors in [172] demonstrated an InP based integrated comb generator consisting of MZI in an amplified ring. The device uses a MZI in a quadrature to generate the sidebands with an amplified feed-back loop to broaden the spectrum. The phase modulators in the MZI use 3-step asymmetric quantum wells to improve the phase modulation efficiency. Unlike fiber based rings, such integrated solutions are more resistant to environmental instabilities like temperature and can lead to more stable solutions in future. Using this approach, a comb with 6 lines within a $3 \mathrm{~dB}$ spectral window and with a FSR of $10 \mathrm{GHz}$ was successfully generated. The flatness of fiber ring combs involving dual side band (DSB) FS is relatively poor compared to single side band (SSB) FS (discussed in next paragraph). This is because the two sidebands generated in every loop circulation have decaying amplitudes. Optical Serrodyne combs have also been reported in the literature. These combs use sawtooth RF waveforms instead of sinusoidal signal for driving the modulator in the loop [173], [174], [175]. A PM driven by a sawtooth waveform acts as SSB modulator and can provide equal amplitude lines on one side of $\mathrm{CW}$. Table X summarizes the recent efforts in this area.

b) Fiber ring cavities containing IQ modulator based SSB-SC frequency shifter: In the DSB frequency shifting scheme new tones are generated in each circulation on both positive and negative frequencies and overlapping is inevitable. The characteristics of the generated OFC can be influenced, if there are any phase or frequency differences between the overlapped tones. OFC tones generated by a SSB frequency shifting process do not overlap, thus can preserve good characteristics of the seed CW source such as linewidth. Endo et al. demonstrated the first SSB frequency shifted RFS comb using a DD-MZM for sideband suppression and a Sagnac loop for carrier suppression [176]. An OFC with an overall bandwidth of $1 \mathrm{THz}$, consisting of 68 lines within a $20 \mathrm{~dB}$ spectral flatness and a FSR of $12 \mathrm{GHz}$ was generated. Subsequently, integrated IQ modulators were used to replace the DD-MZM and the
Sagnac loop as a frequency shifter. The frequency shifting performance of the IQ modulator has already been discussed in detail in section IV-A4. For SSB RFS combs, IQ modulators are used as EO modulators in the generic scheme shown in Fig. 18 (a) [177]. A light signal from a narrow line-width CW source is supplied to the loop, which is composed of a 50:50 coupler, an IQ modulator, an optical amplifier (OA), a tunable optical band-pass filter (BPF) and a polarization controller. The $\mathrm{OA}$ is used to compensate the optical losses in the loop and BPF is used to set the spectral width of the comb. The I and $\mathrm{Q}$ ports of the IQ modulator are driven by RF clock signals of equal amplitude and $\pi / 2$ phase difference. Both sub-MZM are biased at the 'null' and the PM in the outer MZM is biased to provide $\pi / 2$ or $-\pi / 2$ phase shift. In each circulation, the incoming light signal is shifted in frequency by an interval equal to the RF clock frequency, resulting in multiple tones at the output of the coupler.

The quality of the RFS comb is defined by the low crosstalk (the ratio of the first and third harmonic), high effective OSNR (i.e., TNR) and an optimum flatness. The crosstalk is related to the quality of the frequency shifter and is dependent on the IQ modulator device imperfection and operating conditions. The higher crosstalk results in a lower TNR and also in a poor flatness. TNR is limited by gain competition in the amplifier, dependence of ASE on wavelengths and noise accumulation in successive tones, due to repetitive circulations. A lower TNR limits the number of lines than can be generated in an RFS comb generator. Gain competition and crosstalk also affects the flatness of the comb. Authors in [178], [179], [180], [181] provided a comprehensive theoretical and experimental analysis of RFS comb quality and stability in the presence of modulator and amplifier nonlinearities and investigated underlying factors for crosstalk. If the electric field of the input seed is denoted as $E_{i n}(t)=E \exp \left(j 2 \pi f_{0} t\right)$, the RF drive voltages as $f_{I}=V_{p p} \sin \left(2 \pi f_{m} t\right)$ and $f_{Q}=V_{p p} \cos \left(2 \pi f_{m} t\right)$, the normalized transfer function of the RFS loop is given by [179]:

$$
\left.T=\left[\exp \left(j 2 \pi f_{m} t\right)+b \exp \left(j 2 \pi n_{0} f_{m}\right) t\right)\right] \exp \left(j \varnothing_{R T}\right)
$$

where $b$ and $n_{0}(=-3,-2,-1,0)$ are the normalized coeffi- 
Table X: COMBS BASED ON FIBER RING CAVITIES CONTAINING PM BASED DSB FREQUENCY SHIFTER

\begin{tabular}{|c|c|c|c|c|c|c|c|}
\hline $\begin{array}{l}\text { Sr. } \\
\text { No. }\end{array}$ & Ref. & Year & Target/ Issue Addressed & $\begin{array}{l}\text { No. of } \\
\text { Lines }\end{array}$ & $\begin{array}{l}\text { Carrier } \\
\text { Spacing }\end{array}$ & Flatness & $\begin{array}{l}\text { Application/Technique } \\
\text { improvement }\end{array}$ \\
\hline 1 & [166] & 1993 & $\begin{array}{l}\text { One of earliest works } \\
\text { introducing PM in the loop }\end{array}$ & 54 & Variable & poor & $\begin{array}{l}\text { Theoretical analysis of scheme, } \\
\text { time domain model }\end{array}$ \\
\hline 2 & [164] & 1999 & Recirculating loop with PM & 103 & $1-25 \mathrm{GHz}$ & $40 \mathrm{~dB}$ & $\begin{array}{l}\text { Experimental comb, poor } \\
\text { flatness }\end{array}$ \\
\hline 3 & [167] & 2007 & $\begin{array}{l}\text { Carrier spacing tunability, comb } \\
\text { bandwidth }(1 \mathrm{THz})\end{array}$ & 100 & $6-12.5 \mathrm{GHz}$ & $6 \mathrm{~dB}$ & $\begin{array}{l}\text { Frequency domain numerical } \\
\text { analysis, Experimental comb, } \\
\text { FSR tuning from } 6 \text { to } 12.5 \mathrm{GHz} \\
\text { in } 12.5 \mathrm{MHz} \text { steps }\end{array}$ \\
\hline 4 & [168] & 2007 & $\begin{array}{l}\text { Comb bandwidth }(2 \mathrm{THz}) \text {, phase } \\
\text { noise suppression, extension of } \\
\text { previous work }\end{array}$ & 200 & $6-12.5 \mathrm{GHz}$ & $10 \mathrm{~dB}$ & $\begin{array}{c}\text { Experimental comb, low pahse } \\
\text { noise- } 130 \mathrm{dBc} / \mathrm{Hz} \text { at } 1 \mathrm{MHz} \\
\text { offset, low TNR }\end{array}$ \\
\hline 5 & [169] & 2011 & $\begin{array}{c}\text { Comb bandwidth ( } 2.8 \mathrm{THz}) \text {, } \\
\text { TNR improvement, uses two } \\
\text { PMs in loop }\end{array}$ & 113 & $25 \mathrm{GHz}$ & $5 \mathrm{~dB}$ & $\begin{array}{l}26 \mathrm{~dB} \text { TNR in } 22.6 \mathrm{~nm} \text {, Impact } \\
\text { of EDFA gain }\end{array}$ \\
\hline 6 & [170] & 2011 & $\begin{array}{c}11.2 \mathrm{~Tb} / \mathrm{sec} \text { OFDM transmission } \\
\text { using [169] }\end{array}$ & 112 & $25 \mathrm{GHz}$ & $5 \mathrm{~dB}$ & $\begin{array}{l}11.2 \mathrm{Tbit} / \mathrm{s} \text { optical } \\
\text { PM-QPSK-OFDM signal over } \\
640 \mathrm{~km} \text { SMF- } 28\end{array}$ \\
\hline 7 & [171] & 2011 & $\begin{array}{c}\text { Number of lines, TNR } \\
\text { improvement, double loop PM } \\
\text { RFS }\end{array}$ & 178 & $26 \mathrm{GHz}$ & $\begin{array}{l}\sim 10 \mathrm{~dB} \text { and } \\
3 \mathrm{~dB} \text { with } \\
\text { filter }\end{array}$ & $\begin{array}{l}\text { Uses only one phase modulator } \\
\text { in each loop, standalone comb, } \\
20 \mathrm{~dB} \text { TNR }\end{array}$ \\
\hline 8 & [172] & 2012 & $\begin{array}{l}\text { InP based integrated RFS comb } \\
\text { solution based on MZI and } \\
\text { phase shifters. Stability }\end{array}$ & 6,5 & $\begin{array}{l}10 \mathrm{GHz} \text { and } \\
14 \mathrm{GHz}\end{array}$ & $\begin{array}{l}3 \mathrm{~dB} \text { and } 5 \\
\mathrm{~dB}\end{array}$ & $\begin{array}{l}\text { Experimental comb, No fiber } \\
\text { instabilities, comb tunability } \\
\text { over } 80 \mathrm{~nm}, 3 \text {-step asymmetric } \\
\text { quantum wells to improve the } \\
\text { phase modulation efficiency }\end{array}$ \\
\hline
\end{tabular}

cient and the order of crosstalk component respectively and $\varnothing_{R T}$ is the phase delay per round trip. Considering the BPF in the loop blocks any frequency less than or equal to $f_{0}$ and higher than $f_{N}$, the output of the RFS comb can be expressed as [179], [180]:

$$
\begin{aligned}
E_{\text {final }}(t) \approx E_{\text {in }}(t) & \sum_{n=0}^{N} \exp \left(j 2 \pi n f_{m} t\right) \cdot \exp \left(j n \varnothing_{R T}\right)+E_{\text {in }}(t) . \\
& \sum_{n=1}^{N} C_{n} \exp \left(j 2 \pi n f_{m} t\right) \cdot \exp \left(j n \varnothing_{R T}\right) \quad(20)
\end{aligned}
$$

In (20), the first term on the right-hand side corresponds to the desired tone components i.e., "signal components" $f_{m}$ and the second term corresponds to the "unwanted" crosstalk [178]. $C_{n}$ is the normalized crosstalk co-efficient and can be expressed as [179]:

$$
\begin{aligned}
& C_{n}=n b \exp \left[j\left(1-n_{0}\right) \varnothing_{R T}\right], \quad n<\left(N+n_{0}\right) \\
& C_{n}=\left(N+n_{0}\right) b \exp \left[j\left(1-n_{0}\right) \varnothing_{R T}\right], \quad n \geq\left(N+n_{0}\right)
\end{aligned}
$$

Li et al. provided theoretical investigation on the stability of RFS comb in the presence of modulator and amplifier nonlinearities. Authors established that crosstalk is the limiting factor for the performance of a RFS and that it requires proper adjustment of the applied RF signal strength $\left(V_{p p}\right)$ [178]. The crosstalk is dependent on the relationship between the $\left(V_{p p}\right)$ and the desired number of lines $(N)$ : the higher the $N$ and $\left(V_{p p}\right)$, the larger the crosstalk. Later, the same group extended work by investigating the impact of the crosstalk component originated from i) the intrinsic manufacturing imperfections of the IQM, ii) the deviation of DC bias operation voltage and iii) the imbalance in the amplitude and phase of the RF drive signals [178]. The authors concluded that

1) The intrinsic imperfections of a modulator will result in poor carrier suppression.

2) The deviation from right bias voltage will generate the undesired even-order harmonics affecting the spectral quality of the comb.

3) The imbalance in the power and phase of RF drive signals will produce the undesired odd-order tones.

Tian et al. experimentally analyzed the impact of the above factors and the polarization on the flatness of the RFS based comb and successfully generated 50 lines spaced by 12.5 $\mathrm{GHz}$ and having $2.5 \mathrm{~dB}$ spectral flatness [180]. A modified FS scheme aimed at improving the crosstalk by suppressing the third harmonic, has also been proposed and numerically investigated [182]. Here, one arm of the IQ modulator was driven by three times the $\left(3 f_{0}\right)$ fundamental frequency $f_{0}$.

The effective OSNR defines the quality of the signal, which will be available for further data modulation, if the RFS combs are used as carrier source. The noise from amplifier and crosstalk are limiting factor for effective OSNR. The resultant effective OSNR from OA and the crosstalk components is given by [178], [179] :

$O S N R_{O A}(d B) \approx 58+P_{\text {out }}(d B m)-N F(d B)-L_{\text {total }}(d B)-20 \log N$

$$
\operatorname{OSNR}_{\text {crosstalk }}(d B) \approx-20 \log (|b|)-10 \log \left(N+n_{0}\right)
$$


where $P_{\text {out }}$ is the saturation power of the OA, NF is the noise figure and $L_{\text {total }}$ is the total loss of the components in the loop. The effective OSNR of the multi-carrier source can be expressed by combining the contributions from $\mathrm{OA}$ and crosstalk:

$$
O S N R_{e f f}(d B) \approx 10 \log \left(10^{-\left(\frac{O S N R_{O A}}{10}\right)}+10^{-\left(\frac{O S N R_{\text {crosstalk }}}{10}\right)}\right)
$$

In addition to the effect of seed laser linewidth [183] on the quality of OFC, phase noise characteristics of the comb lines and their relationship with frequency spacing [184] have also been studied. Yu et al. demonstrated a 90 line OFC, having $4.26 \mathrm{~dB}$ amplitude variation, by optimizing the length and gain of the loop using additional variable optical attenuator (VOA) and delay lines [185]. Several approaches focused on improving the TNR and increasing the number of lines have been proposed in the literature. These include injecting multiple seeds [186], [187], constructing multiple loops [188], suppressing noise [189], [190] and implementing phase coherence control [191].

Multiple Seeds: Multi-channel multi-carrier source based RFS scheme was demonstrated by introducing multiple lasers in the loop and creating multiple pass-band profiles using a wavelength selective switch (WSS) [192]. Two comb blocks, generating 28 and 29 lines, were created. In this scenario, N seed lasers can produce $\mathrm{N}$ comb blocks and the width of each block depends on the selection made by the WSS in the loop. The single frequency shifting RFS scheme produces one line per circulation and the number of lines is limited due to a low TNR. Typically up to 50 lines exhibiting a TNR of 20 $\mathrm{dB}$ can be generated. The multi-frequency shifting technique produces $\mathrm{N}$ subcarriers in one circulation, reducing the number of circulations by $1 / N$ [186], [187]. The RFS loop is fed by $\mathrm{N}$ seed lasers and the modulator is driven by $N f_{s}$, instead of $f_{s}$, to avoid overlap of generated tones. Two variants of the multiple seed source have been reported and compared with a typical single frequency shifting RFS. In the first type two standard ECLs were used [186], while in the second, a carrier source consisting of single ECL and an IM, was built using DSB suppressed carrier modulation [187]. Multi-frequency shifting using two ECLs, when compared to the single frequency shifting scheme, provides a $10 \mathrm{~dB}$ improvement, while the single ECL based carrier source provides an $8 \mathrm{~dB}$ improvement in the TNR respectively.

Multiple loops: In the traditional RFS loop either a positive or negative frequency shifting is used. The number of lines can thus be increased by exploiting both shifts using two loops. An IQ modulator provides positive frequency shift in one loop and a negative frequency shift in the other [188]. The drawback of the scheme is that double resources (two IQ modulators, two amplifiers) are required for the two loops. $\mathrm{Li}$ et al. proposed and numerically investigated an alternative scheme, utilizing an IQ modulator as a complementary FS (IQ modulator with two outputs), thus saving one IQ modulator [193]. Nested loops have also been proposed for multiple comb block generation. These have several advantages, such as the ability to use a single FS, tunable BPFs instead of expensive
WSS and avoiding gain competition in amplifiers [194].

Noise suppression and phase coherence control: TNR improvement by noise suppression have also been proposed by using an optical finite impulse response (FIR) filter [189] and an AMZI in the loop [190]. A technique providing phase coherence control in the loop has been proposed, which reduces the nonlinearity in the fiber, which allows an increase in the number of lines [191]. Successful long haul terabit NGI-CO-OFDM PM-QPSK [72], [195] and CoWDM PMRZ-16QAM [196] transmission experiments, employing RFS comb as carrier source, have been reported. Nevertheless, for a long term stable operation of fiber ring RFS combs, a feedback mechanism to maintain cavity length, is essential. Typically, RFS based combs producing more than 50 lines suffer from stability issues.

\section{B. Combs based on nonlinearities in periodically poled Lithium Niobate (PPLN) waveguides}

Optical frequency combs can be generated using Periodically Poled Lithium Niobate (PPLN) waveguides by exploiting nonlinear effects called second harmonic generation (SHG), sum frequency generation (SFG) and difference frequency generation (DFG). It is important to give a brief introduction about PPLN waveguides and second order nonlinear effects present in these devices.

1) Periodically Poled Lithium Niobate (PPLN) waveguides; A brief introduction: The PPLN waveguides are highly efficient devices for nonlinear wavelength conversion processes [197]. High efficiency of second order nonlinear effects in PPLN is due to quasi-phase matching (QPM) condition obtained through periodic poling of Lithium Niobate crystals. Periodic poling [198] consists in applying an electric field to particular crystals, by means of properly spaced electrodes. The field is periodically reversed and it has to be strong enough to cause domain reversal, which implies the periodic compensation of phase mismatch that leads to QPM. The fabrication process for PPLN is shown in the Fig. 19 (a). However, QPM condition is achieved over certain limited bandwidth (typically $1 \mathrm{~nm}$ ) and is not true for any wavelength.

Second order nonlinear processes involve mixing of three electromagnetic waves. The magnitude of the nonlinear response of the crystal is characterized by the $\chi^{2}$ coefficient. When two waves having wavelengths $\lambda_{1}$ and $\lambda_{2}$ propagate inside a $\chi^{2}$ crystal, their interaction produces three interesting phenomena: second harmonic generation, sum frequency generation and difference frequency generation as shown in the Fig. 19 (b).

Second Harmonic Generation (SHG) or Frequency Doubling: In SHG two input photons of signal $S$ having same wavelength $\lambda_{S}$, within QPM band, are combined to generate a third photon at half of their starting wavelength $\lambda_{1 / 2}$ i.e. double frequency, due to interaction between light and periodically poled $\chi^{2}$ crystals.

Sum Frequency Generation (SFG): SFG effect combines two photons at $\lambda_{P}$ and $\lambda_{S}$ to generate a third photon at $\lambda_{S F G}$, where :

$$
1 / \lambda_{S F G}=1 / \lambda_{P}+1 / \lambda_{S}
$$


Table XI: COMBS BASED ON FIBER RING CAVITIES BASED ON SSB-SC FREQUENCY SHIFTER

\begin{tabular}{lll}
\hline Sr. No. & Focus Aspect/ Issue Addressed & Related Works \\
\hline 1 & $\begin{array}{l}\text { First SSB RFS comb, DD MZM as SSB generator } \\
\text { and sagnac loop for carrier suppression }\end{array}$ & {$[176]$} \\
\hline 2 & Proposes SSB RFS comb using IQ modulator. & {$[177]$} \\
\hline 3 & Multiple Seeds Multichannel FS (MCFS) & {$[192],[195]$} \\
\hline 4 & Comb enabled Terabit transmission & {$[72],[181],[195],[196]$} \\
\hline 5 & RFS Comb Stability and quality & {$[178],[183],[191]$} \\
\hline 6 & Crosstalk & {$[179],[182]$} \\
\hline 7 & Flatness & {$[180],[183],[194]$} \\
\hline 8 & Phase noise & {$[184]$} \\
\hline 9 & TNR and lines & {$[185],[186],[187],[188],[190],[189]$} \\
\hline 10 & Multi-frequency shifting & {$[186],[187]$} \\
\hline 11 & Dual loops & {$[188],[193],[194]$} \\
\hline 12 & complexity and cost & {$[193],[194]$} \\
\hline 13 & Noise Suppression & {$[189],[190]$} \\
\hline
\end{tabular}

From energy conservation perspective, one photon at frequency $f_{P}$ and one photon at frequency $f_{S}$ are annihilated by two photon absorption and the atom jumps to higher (virtual) energy level. Subsequently, a photon at $f_{S F G}=f_{P}+f_{S}$ is generated and the atom drops back to original state [199].

Difference Frequency Generation (DFG): In DFG, two input photons at $\lambda_{P}$ and $\lambda_{S}$ are combined to generate an output photon at $\lambda_{D F G}$, where :

$$
1 / \lambda_{D F G}=1 / \lambda_{P}-1 / \lambda_{S}
$$

Suppose $\lambda_{S}>\lambda_{P}$, the higher wavelength photon $\lambda_{S}$ induces the lower wavelength photon $\lambda_{P}$ to divide into two other photons, one at $\lambda_{S}$ and the other at $\lambda_{D F G}$.

2) Optical Frequency combs using PPLN waveguides: Preliminary works on comb generation using PPLN [200] were based on oscillators with low repetition rates (less than $200 \mathrm{MHz}$ ) and their suitability for applications in optical communications was not investigated. Scaffardi et al. made first demonstration of PPLN based optical frequency combs for optical communication applications [201]. The working principle is shown in Fig. 20. The $N$ seed optical signals (originated from multiple $\mathrm{CW}$ lasers or a seed generator), placed across QPM frequency within the PPLN QPM band, are fed to PPLN. The nonlinear interaction of these seed signals within PPLN produces SHG and SFG. The generated lines are shifted to $\mathrm{C}$ band exploiting DFG, by placing another pump laser $\mathrm{CW}_{\mathrm{e}}$ at an appropriate frequency. As a result, a comb of optical lines is generated due to SHG and SFG cascaded with DFG. The number of lines can be increased by adding seed lines within the QPM bandwidth. The spacing between the seed lines determines the spacing between the output comb lines. The central tuning or shifting of the comb spectrum is achieved by tuning $\mathrm{CW}_{\mathrm{e}}$, which defines where DFG lines have to be in the output spectrum. The number and spacing between seed lines determine the number of lines, which in turn are dependent on QPM bandwidth of PPLN (typically $~ 1$ $\mathrm{nm})$. The QPM bandwidth limits the achievable carrier/line spacing and overall bandwidth of the generated comb. Authors in [201] also proved that non-uniform spacing between seed lasers decreases overlapping contributions of SFG and SHG, thus increasing the number of lines (for uniform spacing, $N$ seed lines can produce $2 N-1$ lines). In the experiments described in [201], the seeds are sourced from independent lasers, thus resulting in a comb, whose lines were not coherent. Vercesi et al. [202] introduced a seed generator based on a nonlinear element, such as a MZM driven by an RF signal. The PPLN was fed with frequency and phase locked seed generators, instead of independent lasers, thus resulting in a coherent comb, with FSRs of $25 \mathrm{GHz}$ and $37.5 \mathrm{GHz}$. In addition the work also demonstrated efficient multicasting using PPLN based combs, as shown in the Fig. 21. The generated multicast replicas are frequency locked and require less number of pumps than previous multicasting schemes based on PPLN and other technologies. The same group also demonstrated software controlled hitless dynamic reconfiguration of multi-wavelength source based on PPLN [203]. The carrier spacing of modulated signals was reconfigured from $37.5 \mathrm{GHz}$ to $12.5 \mathrm{GHz}$ in $0.5 \mathrm{GHz}$ steps in $100 \mathrm{sec}$ and error free transmission was demonstrated during the whole process. The overall reconfiguration time includes reconfiguration time for RF synthesizer, filters (WSS) and the operating voltage of the DPSK demodulator. The authors also determined the frequency steps for different BER regimes, e.g., sweeping time can be reduced to $55 \mathrm{sec}$ if allowed BER is FEC limit $\left(<10^{-3}\right)$ and larger $1.25 \mathrm{GHz}$ frequency steps are used. Table XII summarizes the state of the art showing contributions of each work.

\section{Combs based on gain switched lasers (with and without external injection)}

Optical frequency combs can be generated by using a direct modulation technique called gain switching. It entails the use of an amplified sine wave or electrical pulse to directly modulate a DC biased semiconductor laser. Several types 
Table XII: COMBS BASED ON PPLN WAVEGUIDES

\begin{tabular}{|c|c|c|c|c|c|c|c|}
\hline $\begin{array}{l}\text { Sr. } \\
\text { No. }\end{array}$ & Ref. & Year & Target/ Issue Addressed & $\begin{array}{l}\text { No. of } \\
\text { Lines }\end{array}$ & $\begin{array}{l}\text { Carrier } \\
\text { Spacing }\end{array}$ & Flatness & $\begin{array}{l}\text { Application/ Technique } \\
\text { improvement }\end{array}$ \\
\hline 1 & [200] & 2011 & $\begin{array}{l}\text { Supercontinuum } \\
\text { generation in PPLN }\end{array}$ & $\begin{array}{l}1350 \\
\mathrm{~nm}- \\
2800 \\
\mathrm{~nm}\end{array}$ & $72 \mathrm{MHz}$ & $40 \mathrm{~dB}$ & $\begin{array}{l}\text { Experimental demonstration, not } \\
\text { suitable for telecom applications }\end{array}$ \\
\hline 2 & [201] & 2014 & $\begin{array}{l}\text { Flexible PPLN based } \\
\text { comb for Optical comm., } \\
\text { FSR tunability }\end{array}$ & 19 & $\begin{array}{l}20 \mathrm{GHz} \text { and } \\
25 \mathrm{GHz}\end{array}$ & $15 \mathrm{~dB}$ & $\begin{array}{l}\text { Experimental comb, } 40 \mathrm{~Gb} / \mathrm{s} \\
\text { DQPSK transmission, central } \\
\text { wavelength tunability, line-width } \\
\text { characterization }\end{array}$ \\
\hline 3 & [202] & 2014 & $\begin{array}{l}\text { Coherent comb output, } \\
\text { Multicast using PPLN } \\
\text { comb lines }\end{array}$ & 5 & $\begin{array}{l}25 \mathrm{GHz} \text { and } \\
37.5 \mathrm{GHz}\end{array}$ & $4.5 \mathrm{~dB}$ & $\begin{array}{l}\text { Seed generator instead of } \\
\text { independent lasers enabling } \\
\text { coherent comb output, } 2 \mathrm{~N}-1 \text { line } \\
\text { for } \mathrm{N} \text { seed lines, Multicasting } \\
\text { demonstrated }\end{array}$ \\
\hline 4 & [67] & 2015 & $\begin{array}{l}\text { Software controlled } \\
\text { hitless dynamic FSR } \\
\text { reconfiguration }\end{array}$ & 5 & $\begin{array}{l}12.5 \mathrm{GHz}- \\
37.5 \mathrm{GHz}\end{array}$ & $5-15 \mathrm{~dB}$ & $\begin{array}{l}\text { Hitless reconfiguration from } \\
37.5 \mathrm{GHz} \text { to } 12.5 \mathrm{GHz} \text {, optimum } \\
\text { frequency reconfiguration steps } \\
\text { for different BER regimes }\end{array}$ \\
\hline 5 & [203] & 2016 & $\begin{array}{l}\text { Dynamic FSR } \\
\text { re-configuration }\end{array}$ & 5 & $\begin{array}{l}12.5 \mathrm{GHz}- \\
37.5 \mathrm{GHz}\end{array}$ & $5-15 \mathrm{~dB}$ & $\begin{array}{l}\text { Hitless reconfiguration using } \\
\text { commercial } 10 \mathrm{GbE} \text { transceivers }\end{array}$ \\
\hline
\end{tabular}

of commercially available semiconductor lasers (distributed feedback-DFB, Fabry Perot-FP, discrete mode-DM, vertical cavity surface emitting lasers-VCSELs) have been used for gain switched OFC generation. A brief introduction to the technique of gain switching is given below after which a selection of the most relevant work in this area is highlighted. The last section looks at externally injected gain switched lasers as important properties of the gain switched OFC could be improved via external light injection.

1) Gain switched lasers; A brief introduction: Gain switching is a direct modulation technique. It essentially involves modulating the laser diode in conjunction with a DC bias to switch the gain of the laser "ON" and "OFF". The typical process of gain switching can be described as follows; a sine wave, at a desired frequency, is amplified with the aid of a high-power RF amplifier. A bias tee is then used to combine the RF signal with a DC bias to enable gain switching of a commercially available laser. It is important to note that gain switching originated as a pulse generation technique, in the early 1980's [204], [205], [206]. One of the major shortcomings with this proposed direct modulation technique was the large temporal jitter and chirp across the generated pulse. These flaws limited its employability in optical time division multiplexed systems. The issues of temporal jitter and chirp were overcome in the 1990's by employing techniques such as external injection [207] and selfseeding [208] to improve the quality of the pulses. From the mid-2000's the spectral properties of externally injected gain switched lasers began to attract attention as the use of OFCs as coherent multicarrier sources within high-capacity optical communication networks, became prominent. The typical rate equations for semiconductor lasers including stochastic carrier recombination and spontaneous emission suffice to show how an optical frequency comb evolves, starting from the freerunning state, right through the final injection-locked state. The single longitudinal laser field rate equations for the carrier density N and slowly varying field envelope E [209] are:

$$
d N d t=\frac{I(t)}{e(V)}-R(N)-a\left(N-N_{0}\right)|E|^{2}+F_{N}
$$

$$
\left.\frac{d E}{d t}=\frac{1}{2}\left[\left(1-j \alpha_{H}\right) a\left(N-N_{0}\right)\right)-\frac{1}{\tau p}\right] E+j \frac{\alpha_{H}}{2 \tau p} E+k_{c} E_{i n j}+F_{E}
$$

The symbols have their usual meaning and are defined in Table XIII. E is normalized such that $|E|^{2}$ is the optical power. The carrier recombination term is $R(N)=A N+B N^{2}+C N^{3}$. External injection is given by the $k_{c} E_{i n j}$ term, $E_{i n j}=E_{i n j^{\prime}}$ $\exp \left(j 2 \pi \Delta f_{\text {det }}\right)$, where $E_{i n j}$ is the field for the master laser obtained by solving (1) and (2) only considering applying a DC current and without external injection. The detuning $\Delta f_{\text {det }}$ is the frequency difference between the $\mathrm{CW}$ master and $\mathrm{CW}$ slave lasers.

\section{Table XIII: LASER PARAMETERS}

\begin{tabular}{ccc}
\hline Symbol & Quantity & Value and Units \\
\hline A & Non radiative carrier lifetime & $1 \times 10^{9} \mathrm{~s}^{-1}$ \\
B & Bimolecular recombination coefficient & $1 \times 10^{-16} \mathrm{~m}^{3} / \mathrm{s}$ \\
$\mathrm{C}$ & Auger recombination coefficient & $1 \times 10^{-41} \mathrm{~m}^{6} / \mathrm{s}$ \\
$a$ & Differential gain & $8 \times 10^{-13} \mathrm{~m}^{3} / \mathrm{s}$ \\
$\alpha_{H}$ & Linewidth enhancement factor & 4 \\
$N_{0}$ & Carrier density concentration at transparency & $1 \times 10^{24} \mathrm{~m}^{-3}$ \\
$\mathrm{~V}$ & Volume of active region & $6 \times 10^{-17} \mathrm{~m}^{3}$ \\
\hline
\end{tabular}

2) OFC Generation using a Gain Switched Single Mode Laser: The first demonstration of this technique was in 2009, where a discrete mode (DM) laser was gain switched, as depicted in Fig. 22 (a), to show an OFC with an FSR of 10 $\mathrm{GHz}$ with 13 lines within a $10 \mathrm{~dB}$ spectral window [210]. The modulation bandwidth of the DM laser was limited, hence the number of lines and flatness of the generated OFC were poor as shown in Fig. 22 (b). However this demonstration 

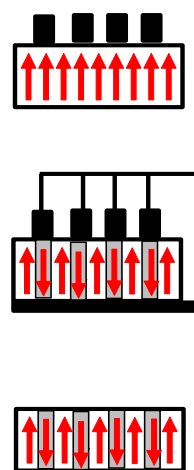

Apply electrodes

to LiNbO3

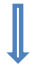

Apply high voltage to periodically poled LiNbO3

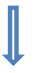

Remove electrodes to produce PPLN

(a)

SHG: second harmonic generation
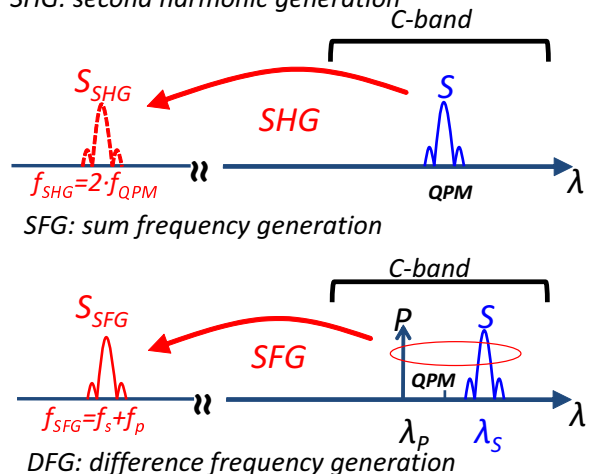

DFG: difference frequency generation

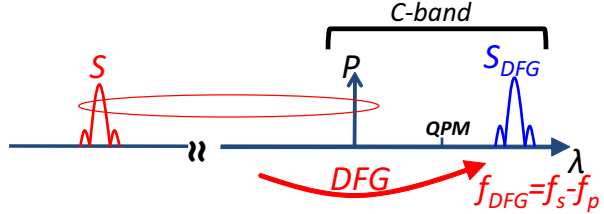

Figure 19: (a) Fabrication process of PPLN [198](b) S- signal, P- Pump signal, QPM- quasi phase matching, SHG, second harmonic generation (top), SFG, sum frequency generation (middle), DFG, difference frequency generation (bottom).

involved the generation of optical pulses with a corresponding frequency comb in the spectral domain. The modulation bandwidth of the DM laser was limited, hence the number of lines and flatness of the generated OFC were poor as shown in Fig. 22.

One of the first implementations of the gain switched DM laser based OFC, in a $10.7 \mathrm{~Gb} / \mathrm{s}$ WDM passive optical access network was reported in [211]. The comb source generated eight tones within a power variation of $1.3 \mathrm{~dB}$. The tones were then individually filtered and modulated (DPSK) prior to transmission over $50 \mathrm{~km}$ of SSMF. Error free performance was reported for each of the eight modulated comb lines with a maximum variation of $1.4 \mathrm{~dB}$ in receiver sensitivity between the channels. This work was then furthered by Maher at al. [212], where authors used the gain switched DM laser based comb source in a Coherent WDM (CoWDM) system. Here again 8 clearly resolved $10.664 \mathrm{GHz}$ coherent tones within 3 $\mathrm{dB}$ of the spectral envelope peak were generated. 7 sub-carriers with a flatness of $2 \mathrm{~dB}$ (improved via filtering) were modulated resulting in a $74.648 \mathrm{~Gb} / \mathrm{s}(7 \times 10.664)$ DPSK CoWDM signal

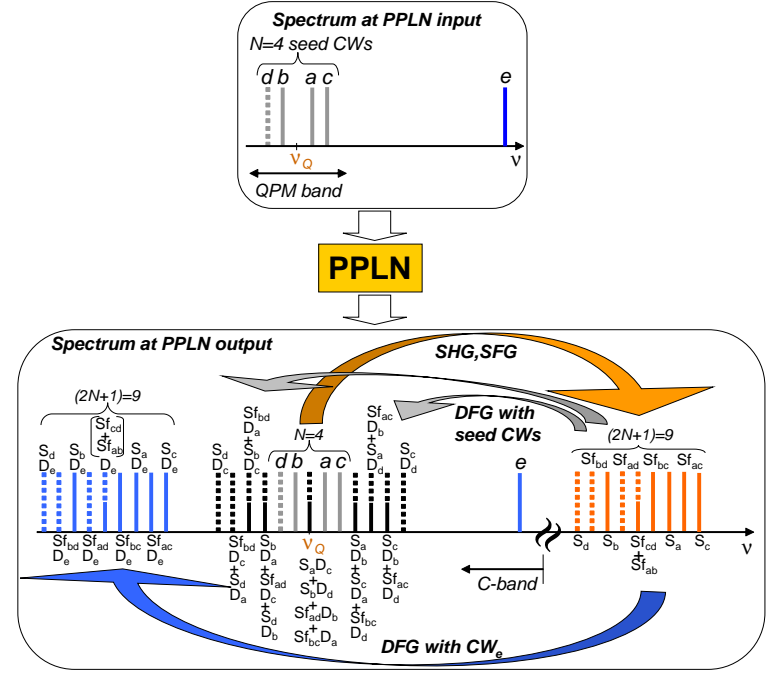

Figure 20: PPLN comb working principle. Si: line generated by SHG of CWi. Sfjk: line generated by SFG of $\mathrm{CWj}$ and CWk. SiDj: DFG of SHG line Si with CWj. SfjkDy: DFG of SFG line Sfjk with CWy. $v$ : QPM frequency of the PPLN. SfjkDy and SiDj lines overlapped with seed CWs are not labeled. CWd, the lines generated in the PPLN by interaction with CWd are displayed with dots [201].

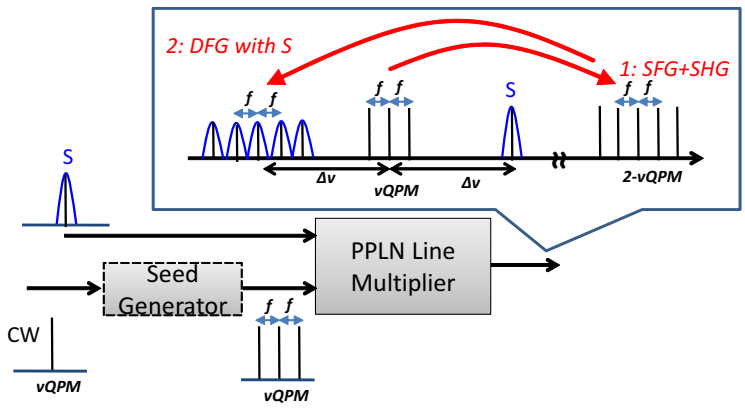

Figure 21: Multicast using PPLN comb, S, signal to be multicast; vQPM, quasi phase matching frequency; $\Delta \mathrm{v}$, wavelength de-tuning with respect to vQPM; f, spacing between comb lines [202].

with a spectral efficiency of $0.64 \mathrm{bit} / \mathrm{s} / \mathrm{Hz}$. Error free operation on each of the sub-channels was reported.

The concept of gain switching a DM laser versus a DFB laser, for comb generation, was then compared by Anandarajah et al. [213]. Here the authors showed DM laser comb with 8 tones separated by $10.7 \mathrm{GHz}$ within $3 \mathrm{~dB}$ of the spectral envelope peak while the modulation (gain switching) of the DFB laser results in a significantly broadened spectrum 23 (a) and (b). As a major constrain associated with this technique is the limited number of tones generated, the authors went on to show some results on comb expansion, where they employed a combination of linear compression with dispersion compensating fibre (DCF) and non-linear compression with HNLF. The frequency spacing and $3 \mathrm{~dB}$ spectral width of the expanded comb were $10.7 \mathrm{GHz}$ and $500 \mathrm{GHz}$, respectively as shown in Fig. 24.

This result was furthered by Latkowski et al. in [214], where 

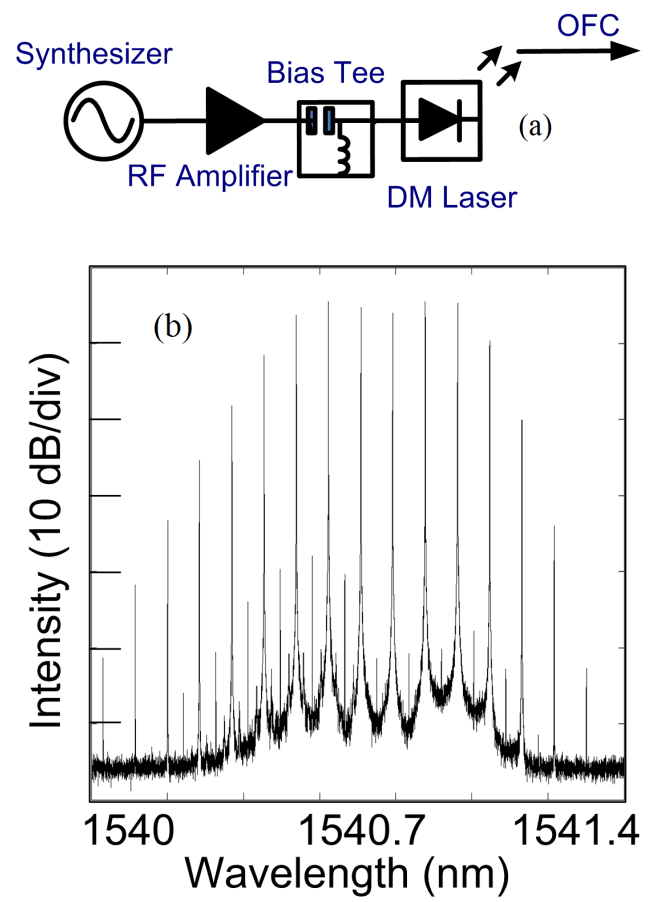

Figure 22: Gain Switching a DM Laser (FSR Tunable OFC) (a) Set-up and (b) optical spectrum [209].
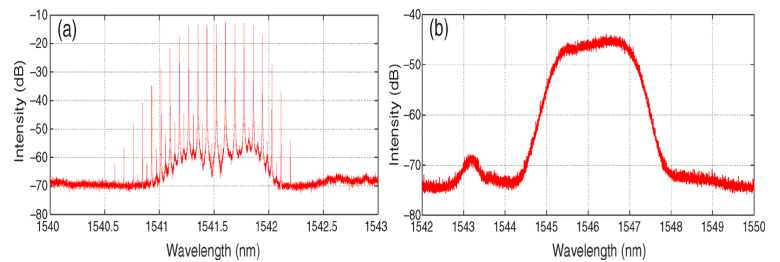

Figure 23: Gain switched optical spectra of (a) DM laser (b) DFB laser.

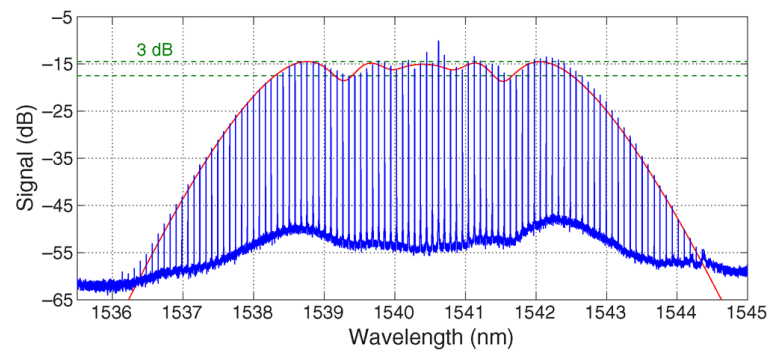

Figure 24: Expanded comb spectrum of the gain-switched DM laser. Measured spectrum (blue trace). Numerical simulation (red trace).

the the gain switched DM comb was expanded by using a phase modulator. Possibilities if simplifying the expansion was also discussed. In 2013, A. R. Criado et al. [215] reported on the use of VCSEL for gain switched comb generation. The authors reported on the generation of a comb exhibiting 20 tones within a $3 \mathrm{~dB}$ ripple at a FSR of $6.25 \mathrm{GHz}$. They went further to beat tones and measure the RF linewidth to exhibit the excellent phase correlation between the tones of the comb. The carrier to noise ratio (CNR) of the comb is not taken into account as the displayed spectrum shows a CNR of only $5 \mathrm{~dB}$ (limited by the OSA). The authors in [216] reported on a gain switched DFB laser that generated a comb with 8 lines within $10 \mathrm{~dB}$ spectral window at an FSR of $15 \mathrm{GHz}$. This paper demonstrated penalty-free alloptical wavelength conversion of 10.7 GBaud QPSK data using four wave mixing and dual-correlated pumps derived from a gain switched comb source having large phase noise characteristics. It showed that the non-degenerate scheme is independent of the phase noise of the dual-pumps as long as they have correlated phase noise. Recently, S. O'Duill et al. [209] presented detailed numerical simulations of the laser dynamics that describe optical frequency comb formation by injection-locking a gain switched laser. They used a standard stochastic laser rate equation model for this work and showed that an extended locking range exists because the comb can be locked by injecting close to the stronger lines of the comb. The locking is corroborated by calculating the FM-noise spectral density, showing that the FM-noise spectral density of the comb lines tends to that of the master as the injection power is increased. Another recent article, in this area from Anandarajah et al. [217] reports on how an optimum OFC could be generated by gain switching a DFB laser close to its relaxation oscillation frequency (ROF) as shown in Fig. 25. The show 9 lines at a FSR of $19 \mathrm{GHz}$ within a spectral ripple of $6 \mathrm{~dB}$. However, the CNR of this OFC is reported to be around the range of $40 \mathrm{~dB}$.

The only implementation of the gain switched DFB comb is reported by Anandarajah et al. in [218]. A six line OFC with each of its lines modulated with 2.5 Gbaud 16-QAM to make a $6 \times 10 \mathrm{~Gb} / \mathrm{s}$ signal is illustrated. The pilot-tone frequency is set at the baud rate and matched filtering is used to recover the high-order QAM symbols in the presence of very high levels of laser phase noise. The 16-QAM signal is then transmitted over an unamplified span of feeder fibre. Subsequently, the pilot tone enables a linewidth independent direct detection scheme at the receiver, thereby enhancing the phase noise tolerance of the system by negating the requirement of a low linewidth tunable LO laser at the receiver and transmitter laser. In general, gain switching of single mode semiconductor lasers provide a cost efficient way of generating an $\mathrm{OFC}$ as it entails the direct modulation of a commercial off the shelf (COTS) laser. In addition, it offers a high degree of flexibility in terms of tuning the FSR achieved simply by tuning the modulation frequency. However, it suffers from limited overall spectral bandwidth, a relatively poor CNR, high linewidth and lack of wavelength tunability (when not externally injected). Table XIV summarizes the reported works.

3) Externally injected gain switched laser (single and multimode): As mentioned earlier, external injection has been reported to improve the quality of the generated comb. One of the first demonstrations of externally injected gain switched lasers (EI GSL) for comb generation was reported by Anandarajah et al. in [213] and is schematically depicted in Fig. 26 (a). This paper reports an OFC exhibiting 19 tones generated within $3 \mathrm{~dB}$ of the spectral envelope peak at a FSR of 10.7 GHz. However, the CNR recorded was about $35 \mathrm{~dB}$. The externally injected gain switched concept was improved by 


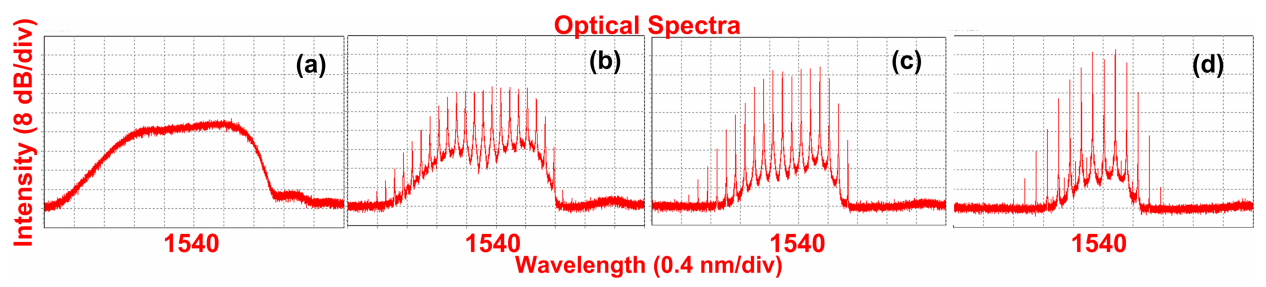

Figure 25: Gain switched DFB laser and it's optical spectra when DFB laser is biased @ $50 \mathrm{~mA} \&$ gain-switched at a frequency of (a) $12 \mathrm{GHz}$, (b) $14 \mathrm{GHz}$, (c) $16 \mathrm{GHz} \&$ (d) $18 \mathrm{GHz}$.

Table XIV: COMBS BASED ON GAIN SWITCHED LASERS

\begin{tabular}{|c|c|c|c|c|c|c|c|}
\hline $\begin{array}{l}\text { Sr. } \\
\text { No. }\end{array}$ & Ref. & Year & Target/ Issue Addressed & $\begin{array}{l}\text { No. of } \\
\text { Lines }\end{array}$ & $\begin{array}{l}\text { Carrier } \\
\text { Spacing }\end{array}$ & Flatness & $\begin{array}{l}\text { Application/Technique } \\
\text { improvement }\end{array}$ \\
\hline 1 & [210] & 2009 & $\begin{array}{l}\text { First demonstration of a gain } \\
\text { switched discrete mode (DM) } \\
\text { laser to generate a pulse/comb }\end{array}$ & 13 & $10 \mathrm{GHz}$ & $10 \mathrm{~dB}$ & Comb alone, Experimental \\
\hline 2 & {$[211]$} & 2010 & $\begin{array}{l}\text { Gain switched DM laser to } \\
\text { generate a comb. }\end{array}$ & 8 & $10.7 \mathrm{GHz}$ & $1.3 \mathrm{~dB}$ & $\begin{array}{l}8 \times 10.7 \mathrm{~Gb} / \mathrm{s} \text { DPSK data } \\
\text { transmission in a PON scenario }\end{array}$ \\
\hline 3 & [212] & 2010 & $\begin{array}{l}\text { Gain Switched DM laser to } \\
\text { generate a comb }\end{array}$ & 8 & $10.66 \mathrm{GHz}$ & $3 \mathrm{~dB}$ & $\begin{array}{c}\text { Comb enables CoWDM system } \\
(7 \times 10.664=74.648 \mathrm{~Gb} / \mathrm{s}), \\
\text { DPSK }\end{array}$ \\
\hline 4 & [214] & 2011 & $\begin{array}{c}\text { GSL comb expansion using } \\
\text { HNLF }\end{array}$ & $8,16,50$ & $10.7 \mathrm{GHz}$ & $3 \mathrm{~dB}$ & Experimental comb \\
\hline 5 & [213] & 2011 & $\begin{array}{l}\text { GSL comb expansion using } \\
\text { linear and nonlinear } \\
\text { compression schemes }\end{array}$ & 8,50 & $10.7 \mathrm{GHz}$ & $3 \mathrm{~dB}$ & Comb alone \\
\hline 6 & [215] & 2013 & $\begin{array}{l}\text { Gain switched VCSEL based } \\
\text { Optical Frequency Combs }\end{array}$ & 20 & $6.25 \mathrm{GHz}$ & $3 \mathrm{~dB}$ & $\begin{array}{c}\text { Comb alone, Phase correlation } \\
\text { investigated }\end{array}$ \\
\hline 7 & [216] & 2014 & $\begin{array}{l}\text { Gain switched DFB laser to } \\
\text { generate a comb }\end{array}$ & 8 & $15 \mathrm{GHz}$ & $10 \mathrm{~dB}$ & $\begin{array}{c}\text { Phase correlation to demonstrate } \\
\text { penalty free wavelength } \\
\text { conversion of } 10.7 \text { Gbaud QPSK } \\
\text { data }\end{array}$ \\
\hline 8 & [209] & 2014 & $\begin{array}{l}\text { Optimum injection conditions to } \\
\text { generate a gain switched optical } \\
\text { comb. }\end{array}$ & - & - & - & $\begin{array}{l}\text { Detailed numerical simulations } \\
\text { of the laser dynamics that } \\
\text { describe OFC formation by } \\
\text { injection-locking a GS laser. }\end{array}$ \\
\hline 9 & [217] & 2015 & $\begin{array}{l}\text { Flexible OFC via gain-switching } \\
\text { of a single-mode DFB } \\
\text { semiconductor laser }\end{array}$ & $10,8,6$ & $\begin{array}{c}14,16,18 \\
\mathrm{GHz}\end{array}$ & $3 \mathrm{~dB}$ & $\begin{array}{l}\text { Numerical analysis and } \\
\text { experimental demonstration, } \\
\text { phase noise }\end{array}$ \\
\hline 10 & [218] & 2015 & $\begin{array}{l}\text { OFC enables multicarrier } \\
\text { transmitter and phase noise } \\
\text { tolerant direct detection }\end{array}$ & 6 & $12.5 \mathrm{GHz}$ & $3 \mathrm{~dB}$ & $\begin{array}{l}\text { UDWDM PON with } 6 \text { X } 2.5 \\
\text { GBaud 16-QAM, } 50 \mathrm{~km} \\
\text { un-repeated downstream } \\
\text { transmission }\end{array}$ \\
\hline
\end{tabular}

Zhou et al. [219], who demonstrated a wavelength tunable OFC source. This OFC was based on the gain switching of an externally injected Fabry Pérot (FP) laser diode and the set-up is illustrated in Fig. 26 (b). In this manner, a highly coherent multi-carrier signal consisting of about 8 clearly resolved 10 $\mathrm{GHz}$ tones generated within $3 \mathrm{~dB}$ of the spectral envelope peak with a CNR in excess of $50 \mathrm{~dB}$ was achieved, and the generated optical comb was shown to be tunable over a wavelength range of $40 \mathrm{~nm}$ (1530 to $1570 \mathrm{~nm}$ ). Two other highlights from this work entails the comb expansion via the use of a phase modulator ( 16 lines in a $3 \mathrm{~dB}$ spectral window) and the transference of the low optical linewidth of the master laser (ECL) to the OFC tones (linewidth recorded as $\sim 100 \mathrm{kHz}$ over the $\mathrm{C}$ band).
This work was furthered by M. D. G. Pascual, et al. in [220], where the tunable comb operation was automated and additional characterisation was carried out. Here, the authors reported on a software reconfigurable, FSR and wavelength tunable gain switched OFC source. This source was based on an externally injected and temperature tuned FP laser diode. It was reported to offer quasi-continuous wavelength tunability over the C-band $(30 \mathrm{~nm})$ and FSR tunability ranging from 6-14 GHz. They demonstrated the spectral quality of the comb tones (RIN $\sim-130 \mathrm{~dB} / \mathrm{Hz}$ and low phase noise of 300 $\mathrm{kHz}$ ). This paper also reported on the stability of the OFC by showing that the fluctuations of the individual comb tones, characterized over 24 hours, were less than $0.5 \mathrm{~dB}$ in power and $5 \mathrm{pm}$ in wavelength. Another report on an EI GSL was 

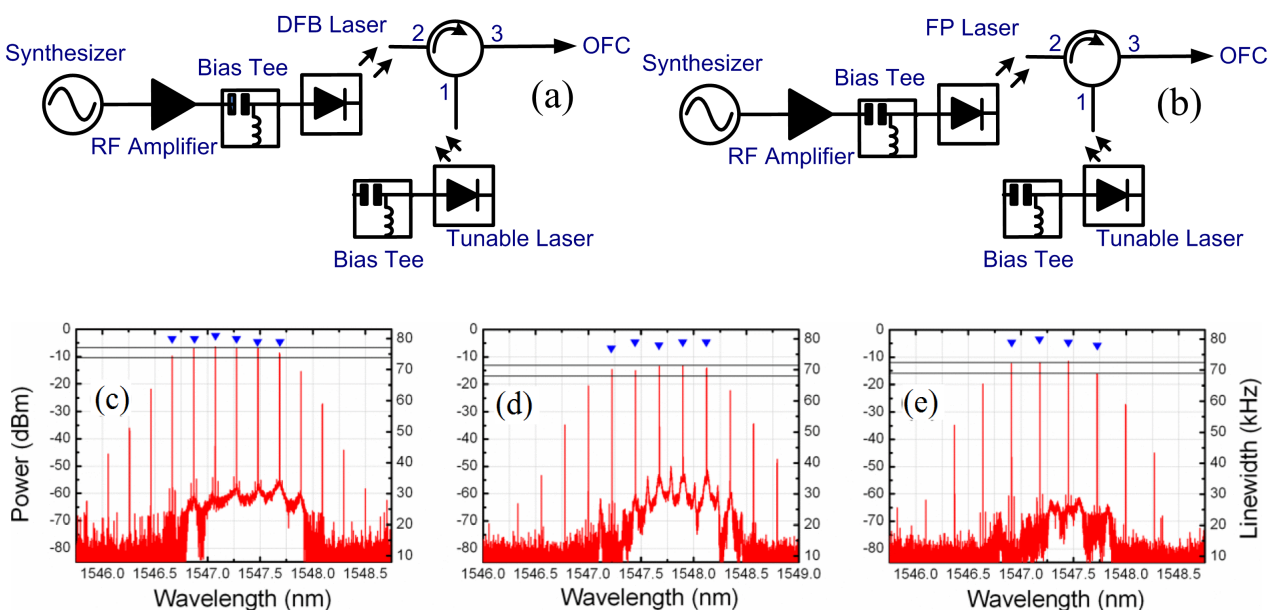

Figure 26: Experimental set-up of (a) gain switching of an externally injected DFB laser (b) gain switching of an externally injected FP laser and Generated combs at the desired FSR (c) $25 \mathrm{GHz}$ (d) $28 \mathrm{GHz}$ (e) $33 \mathrm{GHz}$. Superimposed in blue triangles is optical linewidth of individual comb lines.

from Anandarajah et al. [221], who demonstrated a flexible optical multi-carrier source capable of generating frequency combs at FSRs of 25, 28 and $33 \mathrm{GHz}$. The comb source was realized by simply tuning the injection power and drive frequency. They reported on 6,5 and 4 tones at the respective FSRs as shown in Fig. 26 (c, d and e).

The first system implementation of the EI GSL was reported in [222]. A 7 channel OFC with an FSR of $10 \mathrm{GHz}$ was used with each of tones being modulated with a 3 Gbaud DP-QPSK signal (corresponding to a $10 \mathrm{~Gb} / \mathrm{s}$ data rate). A worst case receiver sensitivity of $44 \mathrm{dBm}$ was reported for a $100 \mathrm{~km}$ unamplified UDWDM PON downlink. The authors go on to show that 14 channels could be used even though the extreme tones would be outside the $3 \mathrm{~dB}$ spectral window. This work was advanced by Zhou et al. in [223], where the comb FSR was increased to $20 \mathrm{GHz}$ with 5 lines used for modulation. A 6 x $40 \mathrm{~Gb} / \mathrm{s}$ (6 Gbaud DP-16QAM) LR DWDM-PON downlink system operating over $80 \mathrm{~km}$ of SSMF was demonstrated with a worst-case channel sensitivity of $-35.6 \mathrm{dBm}$ (54 photons/bit) at a BER of $1.5 \times 10^{-2}$. Another interesting application of the gain switched wavelength tunable comb is reported in [224]. The OFC yielded 7 clearly resolved phase correlated optical tones within a $3 \mathrm{~dB}$ spectral envelope with each of the tones offset by an integer multiple of the drive frequency $(12.5 \mathrm{GHz})$. The $3 \mathrm{~dB}$ optical linewidth of individual comb tones was measured to be $\sim 60 \mathrm{MHz}$ using a delayed selfheterodyne (D-SH) method. A sine wave at $4 \mathrm{GHz}$ supplied to the LO input of the IQ mixer resulted in a 1.25 Gbaud QPSK signal being upconverted to a frequency of $4 \mathrm{GHz}$. The QPSK signal was passed through a $90^{\circ}$ electrical hybrid whose two outputs (offset by $90^{\circ}$ in phase) were then applied to a DD-MZM which resulted in a SSB SCM modulation of the optical comb. The modulated 5 channel SSB-SCMQPSK signal and comb lines (pilot tones) were transmitted over $100 \mathrm{~km}$ with a reported receiver sensitivity of $-20 \mathrm{dBm}$. This pilot tone aided direct detection scheme eliminates the need for a low linewidth optical local oscillator, optical $90^{\circ}$ hybrid and optical phase locking. The first $\mathrm{Tb}$ system using a 9 line EI GSL comb and 16QAM signalling was reported by J. Pfeifle, et al. [225]. Six super-channel architectures that are based on 3 FSR $(20,18.5$ and $12.5 \mathrm{GHz})$ and 2 modulation formats (QPSK and 16QAM.) are verified. The main highlight here entails a net data rate of $1.109 \mathrm{~Tb} / \mathrm{s}$ was achieved for transmission over $150 \mathrm{~km}$ of SSMF using a spectral bandwidth of $166.5 \mathrm{GHz}$, which corresponds to a net spectral efficiency of $6.7 \mathrm{bit} / \mathrm{s} / \mathrm{Hz}$. The net data rate was boosted to $2.112 \mathrm{~Tb} / \mathrm{s}(1.867 \mathrm{~Tb} / \mathrm{s})$ for transmission over $300 \mathrm{~km}$ of SSMF by using a bandwidth of $300 \mathrm{GHz}$ and QPSK modulation on the weaker carriers. The research on externally injected gain switched laser was furthered with reports on an integrated approach. The use of two different monolithically integrated lasers for comb generation via gain switching was first reported by Zhou at al. in [226]. One of the lasers used was a 2-section discrete mode (DM) index patterned laser while the second was a commercially available passive feedback laser (PFL). The 2-section DM laser was gain switched at 5 and $10 \mathrm{GHz}$ resulting in a 9 and 3 line comb (with a $3 \mathrm{~dB}$ spectral ripple) respectively. Subsequently, the authors report on the comb generation with the integrated PFL device at an FSR of 10 and $15 \mathrm{GHz}$ resulting in a 9 and 5 line comb respectively. The PFL comb was then implemented in a $100 \mathrm{~Gb} / \mathrm{s}$ OFDM system by Anandarajah et al. in [227]. Here they realized a 5 optical tone comb source by gain switching the PFL at $12.5 \mathrm{GHz}$. They then intensity modulated each of the 5 tones using single side band direct detection orthogonal frequency division multiplexing (SSB-DD-OFDM). System performance was investigated for propagation distances up to $25 \mathrm{~km}$ of SSMF demonstrating a receiver sensitivity of -15 $\mathrm{dBm}$ without mid-span amplification. M. D. G. Pascual, et al. in [228] proposed a simple potentially integrable configuration for expanding a wavelength tunable comb by cascading gain switched FP lasers. Using such a scheme they demonstrated a $10 \mathrm{GHz}$ spaced comb, exhibiting 13 coherent low-linewidth tones within a $3 \mathrm{~dB}$ window, and tunable over $20 \mathrm{~nm}$. To show that the expanded comb lines exhibited a high degree of phase coherence with each other, the RF beat tone linewidth 
was characterised and shown to have $3 \mathrm{~dB}$ linewidth of 30 $\mathrm{Hz}$ in all cases. M. D. G. Pascual, et al. furthered this work in [229] by simultaneously injection locking two FP longitudinal modes to generate a coherent comb that exhibited a $6 \mathrm{~dB}$ bandwidth of $325 \mathrm{GHz}$ and wavelength tunability over $30 \mathrm{~nm}$. A low optical linewidth $(300 \mathrm{kHz})$ and high coherence between the comb tones was also reported by the authors. Another key application of the EI GSL is a $100 \mathrm{Gbit} / \mathrm{s}$ system implemented as a real-time $5 \times 21.6 \mathrm{Gbit} / \mathrm{s}$ WDM electro-optical transceiver is presented by F. A. Gutiérrez, et al. in [230]. A five line OFC spaced by $20 \mathrm{GHz}$ with each one transmitting four orthogonally overlapping broadband subcarriers where only analogue electronics was employed. The novelty in this report, resides in the analogue implementation where inexpensive microwave IQ mixers and low pass filters permit the orthogonal transmission. A spectral efficiency in purely analogue DD/SCM links was reported, with $1.6 \mathrm{bit} / \mathrm{s} / \mathrm{Hz}$ in the electrical signal and $>1$ $\mathrm{bit} / \mathrm{s} / \mathrm{Hz}$ including the pilot carriers and guard bands. More recent work entails photonic integration of the master-slave lasers used in the gain switched OFCs. In [231], the authors present a photonic integrated device for the generation of flexible gain switched OFCs. The device comprised of two integrated lasers in a master-slave configuration. The spacing between the carriers was adapted by simply modifying the gain switching RF frequency while the number of carriers was altered by modifying the interaction between the two integrated lasers. The FSRs were varied within a range of 6-9 GHz. The main limitation reported here was the limited bandwidth which in turn limited the number of lines. Another negative outcome due to the limited bandwidth is the upper bound placed on the achievable FSR. The externally injected gains switched laser improves the overall bandwidth and also enhances the CNR of the OFC. It is important to note that the complexity of the external injection arrangement can only be mitigated by photonic integration. If this technique were to report on larger bandwidth OFCs, EI-GSLs will prove to be pretty attractive technique. Table XV summarizes the recent works related to EI-GSL combs.

\section{Microring resonator-based comb generator}

1) Introduction: A technique of generating OFCs that has recently gathered research interest, utilizes the Kerr nonlinearity to induce the parametric oscillation in high-Q microcavities. Frequency combs, generated using this method, have been demonstrated in multiple platforms such as silica microtoroids [235], [236], $\mathrm{CaF}_{2}$ resonators [237], [238], $\mathrm{MgF}_{2}$ resonators [239], [240], silica microspheres [241], high-index silica-glass microrings [242] and silicon-nitride microrings [243], [244], [245], [246], [247]. Silicon-nitride microrings are especially attractive, since the resonator and the coupling waveguides can be monolithically integrated. This can lead to a design of an on-chip OFC source that is robust and environmentally isolated. The microresonators are based on a whispering gallery phenomenon, in which certain wavelengths can travel around the ring, building up in intensity over multiple roundtrips due to constructive interference (schematically shown in Fig. 27 (top). The wavelength supported by a ring of a radius $R$ can be calculated using the following equation:

$$
\lambda_{\text {res }} \cdot k=2 \pi \cdot R \quad k \text {-integer }
$$

The light within the ring resonator is guided by total internal reflection at the interface between a dielectric (e.g. semiconductor) material and the surrounding air or distributed Bragg reflection (DBR) from periodical structures. A wide range of microresonator shapes has been explored, with the most widely used being rotationally symmetric structures such as spheres, cylinders, toroids, and disks. Such structures have been shown to support very high-Q whispering-gallery (WG) modes, with modal field intensity distribution concentrated near the dielectric-air interface.
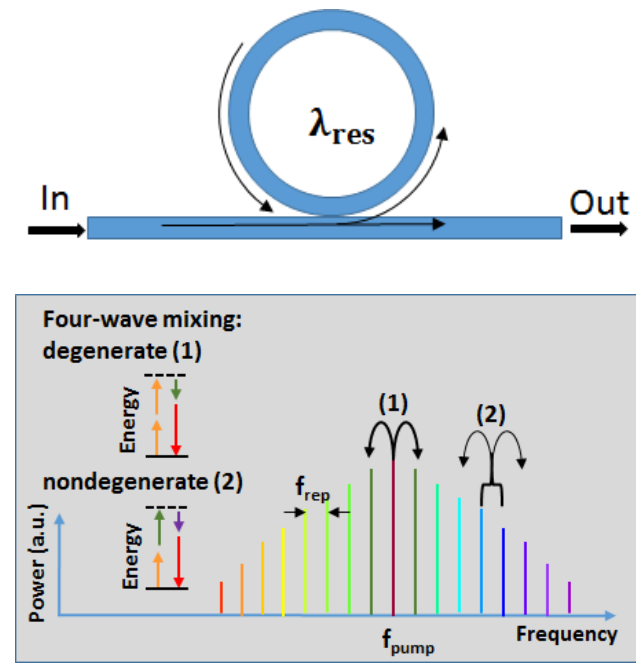

Figure 27: (top) Schematic of a microring resonator; (bottom) Comb generation in ring resonator through degenerate and non-degenerate Four-Wave Mixing.

The comb generation process in the ring resonator is based on the degenerate and non-degenerate FWM process and is instigated by launching a pump signal into the ring. This frequency conversion process originates from the intensitydependent refractive index $n$ :

$$
n=n_{0}+I \times n_{2}
$$

where $n_{2}$ is the Kerr coefficient, $n_{0}$ is the linear refractive index, and I denotes the laser intensity. When a microresonator made from a third-order nonlinearity material is pumped with a CW laser, two pump photons (with angular frequency $\omega_{p}$ ) will be annihilated to create a new a pair of photons: a frequency up-shifted signal $\left.\left(\omega_{s}\right)\right)$, and a frequency downshifted idler $\left.\left(\omega_{i}\right)\right)$. The conservation of energy:

$$
2 h . \omega_{p}=h \omega_{i}+h \omega_{s}
$$

where $h$ is the reduced Planck constant, implies that the frequency components are equally spaced with respect to the pump i.e.:

$$
\omega_{s}=\omega_{p}+\Omega \quad \text { and } \quad \omega_{i}=\omega_{p}-\Omega
$$

If the signal and idler frequencies coincide with optical microresonator modes, the parametric process is enhanced, 
Table XV: COMBS BASED ON EXTERNALLY INJECTED GAIN SWITCHED LASERS

\begin{tabular}{|c|c|c|c|c|c|c|c|}
\hline $\begin{array}{l}\text { Sr. } \\
\text { No. }\end{array}$ & Ref. & Year & Target/ Issue Addressed & $\begin{array}{l}\text { No. of } \\
\text { Lines }\end{array}$ & $\begin{array}{l}\text { Carrier } \\
\text { Spacing }\end{array}$ & Flatness & $\begin{array}{l}\text { Application/ Technique } \\
\text { improvement }\end{array}$ \\
\hline 1 & [219] & 2011 & $\begin{array}{l}\text { First demonstration of an } \\
\text { externally injected gain switched } \\
\text { FP laser based comb }\end{array}$ & $5-8$ & $10 \mathrm{GHz}$ & $3 \mathrm{~dB}$ & $\begin{array}{l}\text { Comb alone, experimental } \\
\text { demonstration of wavelength } \\
\text { tunable OFC }(40 \mathrm{~nm})\end{array}$ \\
\hline 2 & [222] & 2013 & $\begin{array}{l}\text { First demonstration of an } \\
\text { injected GS DFB laser based } \\
\text { flexible comb }\end{array}$ & $6,5 \& 4$ & $\begin{array}{c}25,28 \& 33 \\
\mathrm{GHz}\end{array}$ & $3 \mathrm{~dB}$ & $\begin{array}{c}\text { Shows tunability of FSR by } \\
\text { simply tuning the injection } \\
\text { power }\end{array}$ \\
\hline 3 & {$[221]$} & 2013 & $\begin{array}{c}\text { EI GS DFB laser based OFC } \\
\text { enabled Transmission }\end{array}$ & 15 & $10 \mathrm{GHz}$ & $3 \mathrm{~dB}$ & $\begin{array}{l}3 \text { Gbaud DP-QPSK in a } \\
\text { coherent PON, } 100 \mathrm{~km}\end{array}$ \\
\hline 4 & [223] & 2014 & $\begin{array}{c}\text { EI GS DFB laser based OFC } \\
\text { enabled Transmission }\end{array}$ & 6 & $20 \mathrm{GHz}$ & $3 \mathrm{~dB}$ & $\begin{array}{c}6 \text { Gbaud DP-16QAM } \\
\text { transmission in a CO- PON, } 80 \\
\text { km }\end{array}$ \\
\hline 5 & {$[224]$} & 2014 & $\begin{array}{l}\text { SCM-QPSK Modulation and } \\
\text { Direct Detection }\end{array}$ & 7 & $12.5 \mathrm{GHz}$ & $3 \mathrm{~dB}$ & Experimental demonstration \\
\hline 6 & [228] & 2014 & $\begin{array}{c}\text { Cascaded FP lasers for coherent } \\
\text { expansion of wavelength tunable } \\
\text { GS OFC }\end{array}$ & 13 & $10 \mathrm{GHz}$ & $3 \mathrm{~dB}$ & $\begin{array}{c}\text { Comb only, integrable } \\
\text { configuration for tunable OFC } \\
\text { expanding by cascading GS FP } \\
\text { lasers }\end{array}$ \\
\hline 7 & [225] & 2015 & Carrier spacing tunability & $\begin{array}{c}24,15 \& \\
13\end{array}$ & $\begin{array}{l}12.5,18.5 \& \\
20 \mathrm{GHz}\end{array}$ & $3 \mathrm{~dB}$ & $\begin{array}{c}6 \text { super-channel having variable } \\
\text { FSR (20 GHz, } 18.5 \mathrm{GHz} \text { and } \\
12.5 \mathrm{GHz}) \text { and } 2 \text { modulation } \\
\text { formats (QPSK and 16QAM) } \\
\text { are verified }\end{array}$ \\
\hline 8 & [227] & 2015 & $\begin{array}{l}\text { GS monolithically integrated } \\
\text { passive feedback comb source }\end{array}$ & 4 & $12.5 \mathrm{GHz}$ & $3 \mathrm{~dB}$ & $\begin{array}{l}\text { Integrated comb enabled } \\
\text { transmission, } 5 \times 20 \mathrm{~Gb} / \mathrm{s} \\
\text { WDM-SSB-DD-OFDM } \\
\text { channels, SE } 1.6 \mathrm{~b} / \mathrm{s} / \mathrm{Hz}\end{array}$ \\
\hline 9 & [230] & 2015 & $\begin{array}{l}\text { Comb enabled OFDM } \\
\text { transmission }\end{array}$ & 5 & $20 \mathrm{GHz}$ & $3 \mathrm{~dB}$ & $\begin{array}{l}\text { A real-time } 5 \times 21.6 \mathrm{Gbit} / \mathrm{s} \mathrm{WDM} \\
\text { EO transceiver is presented }\end{array}$ \\
\hline 10 & [229] & 2015 & Broadband flexible OFC & 44 & $6.25 \mathrm{GHz}$ & $3 \mathrm{~dB}$ & $\begin{array}{l}\text { Dual mode injection locking of a } \\
\text { FP Laser for tunable broadband } \\
\text { OFC generation. CNR } 35 \mathrm{~dB}\end{array}$ \\
\hline 11 & [220] & 2015 & $\begin{array}{l}\text { Injection locked GS FP laser } \\
\text { based OFC, Wavelength and } \\
\text { FSR tunability, stability }\end{array}$ & 14 to 6 & $6-14 \mathrm{GHz}$ & $3 \mathrm{~dB}$ & $\begin{array}{c}\text { Comb alone, Software tuning } \\
\text { and control of centre wavelength } \\
\text { tunability is demsontrated. RIN, } \\
\text { linewidth and stability } \\
\text { characterization }\end{array}$ \\
\hline 12 & [232] & 2016 & $\begin{array}{l}\text { OFC enabled real-time } 108 \\
\text { Gbit/s WDM/SCM DSP-less } \\
\text { transceiver }\end{array}$ & 6 & $20 \mathrm{GHz}$ & $3 \mathrm{~dB}$ & $\begin{array}{l}\text { direct detection (DD) } 5 \times 21.6 \\
\text { Gb/s real-time all-analog } \\
\text { WDM/OSCM transmission } \\
\text { scheme. The achieved SE is } \\
\text { higher than in any previously } \\
\text { reported DSP-less WDM/SCM } \\
\text { system }\end{array}$ \\
\hline 13 & [233] & 2017 & $\begin{array}{l}\text { InP photonic integrated EI GS } \\
\text { comb. }\end{array}$ & 9 & $6.25 \mathrm{GHz}$ & $3 \mathrm{~dB}$ & $\begin{array}{c}\text { Comb alone, Experimental } \\
\text { characterization of integrated } \\
\text { OFC }\end{array}$ \\
\hline 14 & [234] & 2017 & $\begin{array}{l}\text { Transmission performance of } \\
\text { integrated gain switched optical } \\
\text { frequency comb source } \\
\text { (GS-OFCS) }\end{array}$ & 9 & $6.25 \mathrm{GHz}$ & $3 \mathrm{~dB}$ & $\begin{array}{c}\text { System1: } 3.125 \text { GBaud PAM-4, } \\
3 \mathrm{~km} \mathrm{SSMF} \mathrm{transmission} \\
\text { System 2: } 5 \text { GBaud Nyquist } \\
\text { QPSK, } 50 \mathrm{~km} \text { SSMF } \\
\text { transmission }\end{array}$ \\
\hline
\end{tabular}

resulting in efficient sideband generation. Next, the generated signal and idler sidebands themselves serve as seeds for further parametric frequency conversion. This phenomenon is also referred to as cascaded FWM or nondegenerate FWM [248]. The non-degenerate FWM causes the creation of multiple equidistant frequency components that can span over $500 \mathrm{~nm}$. The optical comb generation in ring resonator is depictured in Fig. 27 (bottom) [249]. While the ring resonators are capable of generating combs consisting of thousands of lines, they suffer from strong amplitude fluctuations and phase noise. Another drawback of this method is the limited tunability of the comb carrier envelope frequency and the FSR. The first can be adjusted through varying the pump laser frequency with respect to the cavity mode. The FSR on the other hand, can be tuned by using the intensity-dependent round-trip time of the resonator (changing the pump laser power changes the 
refractive index within the cavity through heating as well as the Kerr nonlinearity) [250]. First demonstration of frequency comb generator from a monolithic microresonator was presented by Del'Haye et al. in [235]. The authors reported on the generation of an optical comb by injecting a $130 \mathrm{~mW}$ pump signal at $1550 \mathrm{~nm}$ into a $75 \mu \mathrm{m}$ diameter monolithic toroidal microcavity with a $Q>10^{8}$. The generated OFC consisted of 70 lines, spanning over $400 \mathrm{~nm}(70 \mathrm{THz})$ around $1550 \mathrm{~nm}$. The authors also confirmed the uniformity of the mode spacing to be within a relative experimental precision of $7.3 \times 10^{-18}$.

In [250] same authors demonstrated a control and stabilization of monolithic microresonator-based frequency comb generation. The device used was a $750 \mu \mathrm{m}$ diameter monolithic toroidal microresonators made of fused silica, pumped with $200 \mathrm{~mW}$ laser at $1569 \mathrm{~nm}$ and generating a comb with a mode spacing of $86 \mathrm{GHz}$. Since the generated phase coherent modes occur at frequencies $f_{m}=f_{C E O}+\mathrm{m} f_{\text {rep }}$ (where $\mathrm{m}$ is the number of the comb mode, frep is the mode spacing and $f_{C E O}$ is the offset frequency, given by the carrier envelope offset frequency), there are two degrees of freedom that can be used to control and stabilize a comb: the comb mode spacing and carrier envelope offset frequency. In their experiments, the authors adjusted the frequency of the pump laser in order to control the central mode of the comb. The pump laser was phase-locked to an optical reference defined by a mode of a fully stabilized erbium fiber laser-based comb with a repetition rate of $100 \mathrm{MHz}$. The second degree of freedom, the comb frequency spacing, was controlled by beating the comb modes on a photodiode to produce an RF signal at $86 \mathrm{GHz}$. This was further downconverted to $30 \mathrm{MHz}$, by mixing with the 6th harmonic $(14.3 \mathrm{GHz})$. A phase-locked loop was then used to stabilize the RF signal, thus the frequency spacing of the comb, by adjusting the power of the pump laser. This type of stabilization was highly robust as it did not require any moveable parts and allowed for the mode spacing to be locked to a frequency reference.

Authors in [238] demonstrated the generation of a tunable optical comb in in $\mathrm{CaF}_{2}$ resonators. This was achieved by selecting the proper detuning of the carrier frequency of the pump laser, with respect to a selected resonator frequency. The tuning of the mode spacing with a step of $25 \mathrm{GHz}$ (corresponding to the FSR of the resonator) was presented. The comb spectral width exceeded $30 \mathrm{THz}$ and the lines exhibited very good coherence as proved by the low $(40 \mathrm{~Hz})$ linewidth of the modes beat tone. The flatness of the comb however was relatively poor, with the power difference between lines exceeding $20 \mathrm{~dB}$.

A comb with a record low FSR of $13 \mathrm{GHz}$, using a fluorite whispering gallery mode resonator with the $Q$ factor of $6 x 109$, was demonstrated in [237]. The authors also showed an example of mixed generation, where two combs with different FSR (one FSR being a multiple of the other) were generated simultaneously. The authors attributed this generation to the intensity of Stokes components being strong enough to generate its own spectral comb. Selection of the generation regimes can be determined by coupling conditions and pump power values.

The first monolithically integrated CMOS-compatible comb based on a silicon nitride ring resonator on silicon was presented in [243]. The authors chose the silicon nitride as the material for their resonator, which has a nonlinear refractive index an order of magnitude larger than silica. The advantage of this material is that it allows for a lower $Q$ of the resonator and is CMOS-compatible. By changing the size of the resonator the FSR of choice can be designed. Two devices of different size were demonstrated: one with $58 \mu \mathrm{m}$ radius and $Q$ of 500.000 generated 87 lines spanning S, C, L and $\mathrm{U}$ bands $(1450-1750 \mathrm{~nm})$ and a second smaller one $(20 \mu \mathrm{m}$ radius and $\mathrm{Q}$ of 100.000), which generated 21 wavelengths over a $200 \mathrm{~nm}$ span, with a spacing of $9.5 \mathrm{~nm}$. The authors measured the phase noise of a single mode to be $424 \mathrm{kHz}$. This value was larger than the linewidth of the pump source (140 $\mathrm{kHz}$ ), which the authors attributed to the effect of cascaded FWM process causing the comb generation. In [251] same authors demonstrated the use of their silicon nitride frequency comb in a WDM system. The comb was generated by $116 \mu \mathrm{m}$ resonator and consisted of over 100 new wavelengths spanning across $200 \mathrm{~nm}$. In the experiment, the output of the resonator was collimated and coupled into a fiber. After removing the pump signal, amplification and filtering, 6 individual lines were externally modulated with $10 \mathrm{~Gb} / \mathrm{s}$ data signal. The BER below $10^{-9}$ was measured on the detected lines in a backto-back scenario. In addition, a BER for one of the lines, transmitted over $10 \mathrm{~km}$ of fiber, was also measured and showed less than $0.5 \mathrm{~dB}$ penalty vs. the back-to-back value.

The first demonstration of a coherent transmission using a microresonator comb was presented in [252]. In this publication, the authors used a feedback loop to constantly adjust the power, wavelength and polarization of the pump signal, in order to minimize the phase noise of the generated comb. This was based on the 2 step approach: firstly, by measuring the pump power at the output of the resonator and secondly by observing the modes beat tone on the RF spectrum analyser and maintain a single narrow tone. Two experiments, using two microresonator, were described: one with a FSR of 17 $\mathrm{GHz}$ and a $Q$-factor of $8 \times 10^{5}$ and the second with a FSR of $25 \mathrm{GHz}$ and a Q-factor of $2 \times 10^{6}$. Firstly, five QPSK channels and one 16QAM channel were generated and modulated at a symbol rate of $14 \mathrm{GBd} / \mathrm{s}$. By the use of polarization multiplexing and Nyquist pulse shaping an aggregate data rate of $392 \mathrm{~Gb} / \mathrm{s}$ was achieved. This corresponded to a net spectral efficiency of $6 \mathrm{bits} / \mathrm{s} / \mathrm{Hz}$ for 16QAM and $3 \mathrm{bits} / \mathrm{s} / \mathrm{Hz}$ for QPSK signals (considering 7\% FEC overhead). All channels are well below the EVM 38\% limits for error-free detection for QPSK, and channel 5 showed a sufficiently good performance for 16QAM transmission (11\% EVM). In the second experiment the authors generated 20 channels carrying the total of 1.44 $\mathrm{Tb} / \mathrm{s}$ and transmitted them over $300 \mathrm{~km}$ of fiber. 20 channels showed the EVM below the threshold of $38 \%$.

A novel regime of Kerr comb generation that uses single dissipative solitons was presented in [253]. In this publication the authors exploit the temporal solitons that exist, when a resonator with second-order anomalous group velocity dispersion is pumped far above threshold. The microresonator used for the demonstration had a FSR of $95.8 \mathrm{GHz}$ and a $Q$-factor of $10^{6}$. Using a $3 \mathrm{~W}$ pump power, a set of channels 
spanning the entire $\mathrm{C}$ and L-band with the amplitude variation of +/-5.4 dB was generated. The device was then used for a data transmission of $21.1 \mathrm{~Tb} / \mathrm{s}$ - a record for a chip-scale frequency comb. In order to achieve such a high rate, the odd and even channels were separated and each line was modulated with 28 GBd PDM-16QAM. The combined signal was then transmitted over $75 \mathrm{~km}$ of SSMF. After the coherent detection and offline processing, the BER of 96 channels was calculated. All but two channels had a BER below the FEC threshold. The failing channels were the two located nearest to the pump signal, which suffered from low optical carrier to noise ratio (OCNR - or TNR as used previously). This was caused by an insufficient attenuation of the ASE noise form the EDFA used for the amplification of the pump signal.

The reduction of the comb phase-noise is presented in [254]. Here the authors use a silicon nitride microresonator with a FSR of $18 \mathrm{GHz}$ and an intrinsic $Q$-factor of $1.3 \times 10^{9}$. By injecting an optical pump power of $2 \mathrm{~W}$, a comb spanning $65 \mathrm{THz}$ and containing 3600 lines within $60 \mathrm{~dB}$ from the maximum power line, was generated. The phase noise of the individual lines was measured using the RF beat tone. The lowest value of phase noise to date $(-130 \mathrm{dBc} / \mathrm{Hz}$ at $1 \mathrm{MHz}$ offset) was consistently measured across the comb span. The requirement for a high pump power in order to generate a comb in a microring resonator was addressed in [255]. Here the authors presented a $600 \mathrm{~nm}$ thick $\mathrm{SiN}$ microresonator with an intrinsic $Q$ of 17 million and FSR of $25 \mathrm{GHz}$. The comb initiation power in the device was shown to be as low $5.6 \mathrm{~mW}$, which was attributed to the high $Q$ of a resonator (the pump power decreases quadratically with increasing $Q$ ). The stateof-the-art of the microring resonator-based combs generation in summarized in Table XVI.

\section{COMPARATIVE ANALYSIS OF DIFFERENT OFC GENERATION TECHNIQUES}

This section provides a comparative analysis of different OFC generation techniques, presented in the previous section, in terms of features required by flexible optical carrier sources (outlined in section II-E). The choice of combs and its ideal characteristics is dependent on the target application and no single solution fits all situations. It can be observed from this survey that optical frequency combs based on EO modulation (single or multiple EO devices) dominate the multicarrier generation techniques. The EO comb generators can provide carriers with desired optical characteristics and are flexible, integrable and scalable. Techniques providing few to tens and hundreds of lines have been demonstrated and their transmission performance has also been evaluated. However, high power and broadband electrical amplifiers are required to drive modulators for higher conversion efficiency and the carrier spacing is limited to less than $50 \mathrm{GHz}$. OFCs based on single EO device (such as single and dual drive MZMs, IQ modulator) can provide 8 to 10 lines and are simple, cost effective and power efficient. As such, they are best suited for modular transceivers in real systems. The spectral bandwidth of the OFC can be increased by cascading similar or different types of multiple EO devices. Cascaded IM and PM is the most popular technique that can provide ultra-flat OFC with tens of lines. Spectral bandwidth up to $1071 \mathrm{GHz}$ (enough to generate 28 lines spaced at $37.5 \mathrm{GHz}$ ) have been demonstrated. Several integrated OFCs have also been reported. Similarly, cascaded IMs also increase spectral bandwidth, provide better rectangular shape due to good flatness $(<1 \mathrm{~dB})$ and high SMSR. However, the number of lines for the larger carrier spacing are limited (approximately 9) compared to cascaded IM and PM schemes. The intensity modulators also require bias stabilizers.

OFC using PMs provide comparable number of lines, but are more stable, have smaller insertion loss and footprint and require no DC bias. EO combs have been demonstrated using $\mathrm{InP}, \mathrm{LiNbO}_{3}$ and silicon organic hybrid ( $\left.\mathrm{SOH}\right)$ devices. The improvement in the material system enhances the modulation index and hence comb quality and power efficiency.

Similar to PMs, PolMs also do not require DC bias control, hence provide more stable operation of combs. The typical operational frequency range for PolMs is $40 \mathrm{GHz}$, which can ensure larger overall OFC bandwidth and frequency spacing. The use of PolM for flexible OFC generation is relatively new and has several open issues to be addressed and future research possibilities. Despite several promising works reported above, no transmission characterization of PolM based OFCs has been performed yet (to the best of our knowledge). It is also important to ascertain the cost and complexity of opto-electronic oscillators (OEO). Moreover, the integration of polarization controllers, polarizers and PolMs on a single platform can be challenging and a fully integrated OFC solution based on PolM has not been reported so far.

RFS combs increase the spectral bandwidth by repeated frequency shifting in the fiber ring without using multiple modulators in cascade. The flatness of RFS combs containing PMs is relatively poor because two sidebands generated in each circulation have decaying amplitudes. The stability of the generated OFC can be degraded if there are any phase or frequency differences between the overlapped tones. The overlapping of tones is absent in IQ modulators based rings, which rely on SSB frequency shifting, but bias controllers are required to cater for bias drifting issues. In SSB FS combs, the main limiting factor, affecting the comb quality, is crosstalk. It is related to the quality of the frequency shifter and is dependent on the IQ modulator device imperfection and operating conditions. The number of lines, in both types, is limited by accumulation of noise from the EDFA, which reduces the TNR. Nevertheless, a feedback mechanism is essential to maintain cavity length for long term stability of the comb. The photonic integration can help to overcome stability issues. Another limitation of cavity based combs is the limited range, over which $\mathrm{CW}$ can be tuned.

Parametric combs exhibit flexibility, wide bandwidth (over $100 \mathrm{~nm}$ ), high coherence (narrow line-width $\sim 10 \mathrm{kHz}$ ). The cost and power consumption for each line is significantly lower than conventional lasers. The performance of such OFCs have been validated by transmission experiments. Dynamic transceiver reconfiguration has also been demonstrated. Larger than $50 \mathrm{GHz}$ carrier spacing can also be provided with an additional pre-chirping section and phase synchronized seed 
Table XVI: COMBS BASED ON MICRORING RESONATORS

\begin{tabular}{|c|c|c|c|c|c|c|c|}
\hline $\begin{array}{l}\text { Sr. } \\
\text { No. }\end{array}$ & Ref. & Year & Target/ Issue Addressed & $\begin{array}{l}\text { No. of } \\
\text { Lines }\end{array}$ & $\begin{array}{l}\text { Carrier } \\
\text { Spacing }\end{array}$ & Flatness & $\begin{array}{l}\text { Application/Technique } \\
\text { improvement }\end{array}$ \\
\hline 1 & {$[235]$} & 2007 & $\begin{array}{c}\text { First demonstration of frequency } \\
\text { comb generator from a } \\
\text { monolithic microresonator }\end{array}$ & 70 & $875 \mathrm{GHz}$ & $\begin{array}{l}38 \text { lines } \\
\text { within } 10 \\
\text { dB }\end{array}$ & Comb alone \\
\hline 2 & {$[250]$} & 2008 & $\begin{array}{c}\text { Stabilization and control of FSR } \\
\text { and central wavelength of the } \\
\text { comb }\end{array}$ & 29 & $86 \mathrm{GHz}$ & $\begin{array}{l}20 \text { lines } \\
\text { within } 20 \\
\quad \mathrm{~dB}\end{array}$ & $\begin{array}{l}\text { Comb and millimeter wave } \\
\text { generation }\end{array}$ \\
\hline 3 & [238] & 2008 & $\begin{array}{l}\text { Generation of tunable optical } \\
\text { combs }\end{array}$ & - & $\begin{array}{l}\text { Multiples of } \\
25 \mathrm{GHz}\end{array}$ & - & Comb and mmWave generation \\
\hline 4 & {$[237]$} & 2009 & $\begin{array}{l}\text { MRR based OFC with record } \\
\text { low repetition rate of } 13 \mathrm{GHz}\end{array}$ & $5-8$ & $\begin{array}{c}13 \text { to } 359 \\
\mathrm{GHz}\end{array}$ & $20 \mathrm{~dB}$ & Experimental comb \\
\hline 5 & {$[243]$} & 2010 & $\begin{array}{l}\text { First monolithically integrated } \\
\text { CMOS-compatible comb based } \\
\text { on a silicon nitride ring } \\
\text { resonator on silicon }\end{array}$ & $21 \& 87$ & $\begin{array}{l}1.17 \mathrm{THz} \& \\
403 \mathrm{GHz}\end{array}$ & $20 \mathrm{~dB}$ & Comb alone \\
\hline 6 & {$[251]$} & 2012 & $\begin{array}{l}60 \mathrm{~Gb} / \mathrm{s} \text { data transmission based } \\
\text { on } 6 \text { comb tones over } 10 \mathrm{~km}\end{array}$ & 6 & $200 \mathrm{GHz}$ & - & Comb and OOK transmission \\
\hline 7 & {$[252]$} & 2014 & $\begin{array}{c}\text { First demonstration of coherent } \\
\text { data transmission using MRR } \\
\text { OFC }\end{array}$ & 5 & $17 \mathrm{GHz}$ & $15 \mathrm{~dB}$ & Comb and coherent transmission \\
\hline 8 & {$[253]$} & 2015 & $\begin{array}{l}\text { First demonstration of a } \\
\text { cavity-soliton Kerr comb used } \\
\text { for data transmission }\end{array}$ & 94 & $95.8 \mathrm{GHz}$ & $15 \mathrm{~dB}$ & Comb and data transmission \\
\hline 9 & {$[254]$} & 2015 & $\begin{array}{l}\text { Lowest phase noise floor } \\
\text { achieved to date }\end{array}$ & 3600 & $18 \mathrm{GHz}$ & $60 \mathrm{~dB}$ & Comb characterization \\
\hline 10 & {$[255]$} & 2015 & $\begin{array}{l}\text { MRR OFC with record low } \\
\text { pump power of } 5.6 \mathrm{~mW}\end{array}$ & 3 & $25 \mathrm{GHz}$ & $40 \mathrm{~dB}$ & Demonstration of device \\
\hline
\end{tabular}

lasers. These distinguishing features make parametric combs an attractive choice for replacement of lasers as carriers. The integrated solutions for parametric combs could become strong candidates for future systems e.g. SDM networks, as demonstrated recently [256], where number of carriers needed will increase multi-fold.

Both RFS and parametric combs provide large number of carriers. However, transponder boards in commercial systems are modular and compact, with each transponder board having its own dedicated carrier source (lasers). Thus the use of combs in current node architecture will not be feasible from cost, space and energy efficiency perspective. They could however be considered for application in other architectures, where a centralized carrier source is shared by all the transponders in a node or network. Node and network level considerations will be discussed in detail in the following sections.

Although PPLN based combs are flexible, compact, integrate able, exhibit narrow line-width and support efficient multicasting but they still have certain limitations and further research is needed. The current solutions have poor flatness, low extinction ratio, high power penalty and low line conversion efficiency i.e., ratio of input seeds and output lines is low. Moreover, overall comb bandwidth and achievable FSR is limited by QPM bandwidth of the PPLN. The performance of PPLN devices is sensitive to temperature variations.

Utilization of micro ring resonators for optical frequency comb generation is amongst the newest methods of achieving a MW source. Its unquestionable advantage is the ability to generate a multitude of spectral lines spanning hundreds of nm. In addition, silicon-nitride based microrings lend themselves towards integration with other photonics components. The main disadvantages of this method lies in the need for a high power pump laser necessary to trigger the nonlinear process within the ring. Other challenges include a difficulty in obtaining a good amplitude and phase stability as well as the limited tunability of the FSR.

Direct modulation of a commercial-off-the-shelf (COTS) laser enables the technique of gain switching, for optical frequency comb generation, to demonstrate features such as simplicity and cost efficiency. Other major advantages of the gain switched OFC technique includes low tone linewidth, a high degree of flexibility (FSR and wavelength tunability), excellent stability and small footprint. However, in order to achieve an optimum degree of flatness, number of tones, wavelength tunability and low optical tone linewidth, this technique has to be used in conjunction with external injection. With the use of monolithic or photonic integration, the complexities and shortcomings of external injection can easily be mitigated. Some of the main disadvantages could be the limited number of tones generated (due to the bandwidth of the lasers) and the relatively high power consumption. Obviously the latter disadvantages could be addressed by expanding the comb (nonlinear techniques or passing through cascaded modulators) at the expense of complexity and cost.

The case for feasibility; integrated designs, flexibility demonstrations and field trials:

Table XVII summarizes the key demonstrations of the integrated designs and field trials, which strongly support the 
case for feasibility of MW sources based on OFCs.

Table XVII: FIELD TRIALS AND INTEGRATED DESIGNS

\begin{tabular}{ccc}
\hline Sr. No. & Focus Aspect & Related works \\
\hline 1 & Integrated combs & {$[78],[79],[92],[102]$,} \\
& & {$[151],[172],[226],[244]$} \\
\hline 2 & Transceiver and Network flexibility & {$[66],[108],[53],[125]$,} \\
& & {$[39],[156],[203]$} \\
\hline 3 & Comb enabled Terabit transmission & {$[71],[72],[144],[170]$,} \\
& & {$[181],[195],[196],[225]$,} \\
& & {$[253]$} \\
\hline 4 & Field trials & {$[120],[121]$} \\
\hline
\end{tabular}

\section{OFC DE-MULTIPLEXING/ FILTERING TECHNIQUES}

\section{A. Introduction}

OFC sources have been proven to be very useful for many applications. As each comb line/channel is related to a different wavelength, most of the afore-mentioned applications require channel selection and manipulation. Hence, optical wavelength selection of individual tones (optical filtering/demultiplexing) is of paramount importance. The same applies to DWDM high speed optical communication systems that employ OFCs. De-multiplexing/separation of individual comb tones prior to data encoding is a key operation. Hence, an optical filter, that offers channel selectivity of singlein and multiple-out capability, will be a key component in systems that employ OFCs. This section describes the key features and current technologies for OFC filters. Currently, de-multiplexing of individual channels is one of the major challenges that OFCs face in terms of market adoption. The main parameters that the filter needs to portray include an optimum passband bandwidth, low insertion loss, small footprint, large channel isolation, FSR tuning, low cost and small/no group delay across the filter bandwidth. The stringent requirements and their impact are itemised below:

Low insertion loss of de-multiplexer: Most of the OFCs generated exhibit pretty low per-tone average optical power. Hence, a high insertion loss associated with the de-multiplexer would mean that the filtered carriers need to be compensated by conventional amplification technologies at the expense of degrading the OSNR.

De-multiplexer with small FSR and small footprint: Traditional WDM systems have been using the ITU-T standard grid for channel spacing. In such systems, multiplexing and de-multiplexing of the multi-wavelength channels was carried out by arrayed waveguide gratings (AWG), which satisfied the requirements for FSRs of 200, 100, 50 and $25 \mathrm{GHz}$. However, the next generation systems propose spacing of 12.5 and 6.25 GHz. These denser channel spacing (ultra-dense wavelength division multiplexed-UDWDM) impose a major challenge to the fabrication of optimal filters for the OFC based systems in terms of achieving the stipulated channel isolation. In addition, the decreasing channel spacing (smaller FSR), leads to the footprint of the AWGs getting larger and unmanageable (countering the miniaturization, which integration tries to achieve).
Passband bandwidth of de-multiplexer: Achieving a high finesse filter with a repeating comb like transfer function has proved to be challenging thus far. A low finesse filter employed in UDWDM systems (12.5 or $6.25 \mathrm{GHz}$ spacing) will result in the leakage of neighbouring channels (poor adjacent channel rejection ratio), which in turn will lead to cross channel interference. Optimum channel isolation factors are specified to be $>25 \mathrm{~dB}$.

Temperature stability: The temperature dependence of the waveguide used could cause shifts in the filter bands, thereby resulting in degraded system performance. Obviously, the impact of this effect is worse in UDWDM systems.

Centre wavelength tunability: If the filter is adopted into a system that portrays wavelength tunability, the tuning range of the filter will be required to cover the entire C-band.

There are many different parameters that could be used to classify filters. However, in the context of filter technologies reported in frequency comb experiments thus far and relevant to this review, we have split the filters into two major categories namely; passive and active optical filters. Each of the techniques would have its pros and cons, which can be classified under the stringent specifications highlighted above. It should be noted that this is not an exhaustive list and that the focus of our paper is to highlight the challenges faced in de-mulitplexing of OFCs.

\section{B. Passive optical filters}

There are many types of passive optical filters that have been used in optical communications systems. An overview of some of the most commonly used passive filters is given below. One of the most basic would be the Fabry-Perot (FP) interferometer filter [257]. It's based on a resonant cavity between two mirrors. The device will have high transmittance when the reflections from the mirrors reach the exit at the same phase. The transmittance of an FP filter is a periodic Lorentzian function of frequency. These filters generally exhibit a relatively poor finesse and can also suffer from relatively high insertion loss. Another commonly used comb filter is based on the Mach-Zehnder (MZ) interferometer configuration [179]. Incoming light is split in two ways and recombined after a short distance. The combining fields will interfere according to the phase difference between the two fields. When a time delay is introduced in one arm, a periodic frequency-dependent phase difference is formed. The output intensity is therefore a periodic function on the optical frequency, usually with a small FSR. However, the MZ transfer function suffers from low finesse due to its inherent cosine shape and needs to be cascaded together to form a single-in multiple-out de-multiplexer. The difficulty in obtaining optimum filters has led to quite a lot of the OFC based system demonstrations, within a laboratory environment, using MZ based interferometers or delay interferometers (DIs). They are also commonly referred to as odd and even filters and consist of a single input and two outputs. The fibre Bragg grating (FBG) is another type of filter that is commonly used in photonics [258]. It consists of a periodic perturbation of the refractive index along a length of fibre, which is formed by 
exposure of the core to an intense optical interference pattern. Here again, to achieve a single-in multiple-out de-multiplexer function, several FBGs need to be cascaded together. This results in a very high insertion loss and more importantly an unequal attenuation to the channels. As mentioned earlier, multiplexing and de-multiplexing of the multi-wavelength channels, in traditional DWDM systems, was carried out by AWGs [259]. AWGs enable the simultaneous filtering of the densely spaced channels and have been the widely adopted demultiplexing solution adopted. However, with the decreasing channel spacing (smaller FSR), the footprint of the AWGs gets larger and unmanageable (countering the miniaturization which integration tries to achieve). Another issue is the temperature dependence that could cause shifts in the filter bands thereby resulting in degraded system performance. Yet another problem with the use of AWGs is that it could introduce relatively large losses Another technology that is commonly used to de-multiplex OFCs is a wavelength selective switch (WSS) [260]. Typically, a WSS is realized by combining a quantized dispersion element and an array of switching. The dispersion allows one to focus all the wavelengths within predetermined wavelength bands onto the same location in the switching array. The plurality of switching elements can then be adapted to couple the de-multiplexed beam onto the respective switching element. Most of the reports on WSSs are based on liquid crystal on silicon (LCOS). These LCOS based WSSs are also currently used as the basic building block of ROADM in recent years. In the process of migrating to a higher port count WSS without introducing a complex configuration or loss increase entails the use of planar lightwave circuits (PLCs) [261]. The design and fabrication of siliconbased integrated multichannel filter cascaded by a wavelength de-multiplexer has been reported by numerous groups [262], [263], [264]. The multichannel filtering is achieved by a DI structure and the de-multiplexing is performed by an AWG. Many other methods can also perform the desired functions, such as the dielectric multilayered filters [262], the photonic crystal based drop filter [263], and the plasmonic nanodisk cavities [264]. One of the main issues with this technique is that the channel count in the demonstrations to date are pretty low. The passive techniques usually suffer from high insertion loss, large FSR, temperature stability and a non-flat group delay across the filter bandwidth. The major advantage with this type of filtering is that it involves passive elements; hence is cost and energy efficient. The reconfiguration time of passive filters varies from hundreds of microseconds to tens of milliseconds (based on the technology used). For example; LCOS based WSSs can be tuned in 100s of microseconds while MRRs are tuned in 100s of milliseconds.

\section{Active optical filters}

Much work was done with active filters during the 1980s, which involved the use of a laser (usually below threshold) to allow selective wavelength filtering of input signals. By using more complicated cavity geometry devices such as distributed feedback (DFB) [265] and sampled grating distributed Bragg gratings (SG-DBR) [266] narrowband filtering is achievable with high suppression ( $>30 \mathrm{~dB}$ ) of spurious wavelengths. The active nature of the devices also means that, through carrier injection, the index can be altered resulting in tunability of the filter. Used above threshold, active filters become useful in filtering coherent combs. Through injection locking, the coherence of the filtered wavelengths with the original comb source is retained. This gives active filters potential application in coherent communication system as de-multiplexers. Some of the work in this are focuses on the use of slotted FabryPérot (SFP) semiconductor lasers as active filters [267]. Experiments were carried out to ensure that SFP lasers were useful as tunable active filters. More recent work in this area has been reported by Zhou et al. [268] where the authors present a one-in-four out injection locked comb tone demultiplexer and the use of such a device in a 4-channel $100 \mathrm{~km}$ UDWDM transmission system operating with 12 GBd Nyquist QPSK modulation. They also show experimental results, where stable locking is accomplished with a reasonable residual tone suppression. In addition, they report on the demultiplexer adapting to different input comb tone spacing (6.25 and $12.5 \mathrm{GHz}$ ). This work was then furthered by the same group via an experimental demonstration of mixed symbol rate and modulation format transmission system using the asymmetric/unequally spaced outputs from the injection locked de-multiplexer [269]. More recently, M. D. Gutierrez et al. reported on a four-output monolithically integrated demultiplexer using optical injection locked semiconductor lasers for coherent comb tone separation [231]. In essence, the active technique is based on the principle of injection locking, which translates to disadvantages such as higher power consumption, polarization dependence and temperature instability. However, it lends itself to photonic integration, which can mitigate most of the ill effects mentioned above. Moreover, there are many advantages such as wavelength tunability, optical amplification and maintenance of phase coherence. The active filters entail the use of current to achieve its tuning hence result in shorter reconfiguration times in the order of hundreds of nanoseconds.

\section{NODE AND NETWORK LEVEL CONSIDERATIONS}

The OFC based carrier source can provide better spectral efficiency, transmission performance at physical layer and ease of flexibility compared to the standard lasers. Nevertheless, it is equally important to make sure that the OFC poses no constraints at network layer performance and node architecture. In this section the impact of OFC based carrier sources on the network performance is discussed. Moreover, different possible real network deployment strategies and their impact on node architecture and techno-economics is also presented. Standard lasers do not introduce any constraint on routing and spectrum assignment (RSA): each individual laser can be tuned independently to any wavelength and allocated to any frequency slot in the spectrum. Comb SCGs provide stable frequency locked carriers, but have certain constraints outlined below and depicted in Fig. 28:

Spectral contiguity: the carriers have to be allocated to contiguous frequency slots. If flows are routed in different directions, contiguous spectrum is also needed on different routes, a further constraint during RSA. 

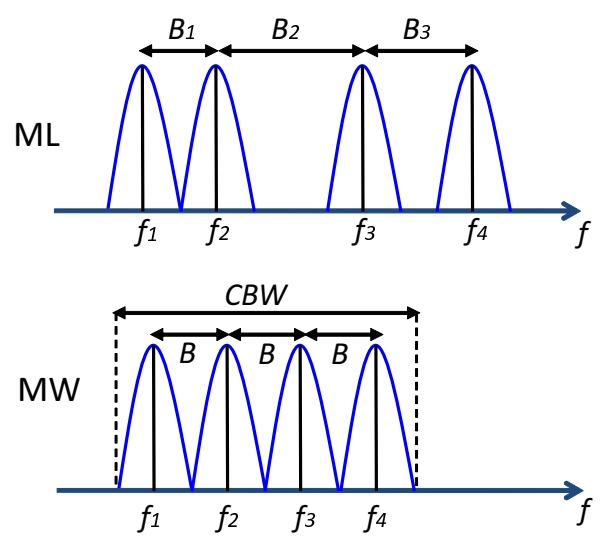

Figure 28: Understanding MW constraints from RSA perspective: (top) Spectrum for carriers based on standard multiple lasers (ML), any value of B can be set. (bottom) Spectrum for Comb-SCG; B, carrier spacing; CBW, comb bandwidth; $\mathrm{f}_{\mathrm{i}}$, central frequency.

Limited carrier spacing: The achievable carrier spacing is limited by electrical bandwidth of EO devices and RF clock sources typically $(\mathrm{B}<50 \mathrm{GHz})$. In case of individual lasers there is no constraint on carrier spacing (within the $\mathrm{C}$ band).

Spectral bandwidth of OFC: The comb spectral bandwidth $(\mathrm{CBW})$ is the overall bandwidth occupied by all the lines of the generated comb. Each OFC can provide spectral quality of carriers only within a certain spectral bandwidth. Typically the $3 \mathrm{~dB}$ bandwidth is considered.

These constraints may result in increased probability of blocking during provisioning and restoration.

\section{A. Impact of MW SCG on network performance}

Dallaglio et al. [270] investigated the impact of SBVTs based on MW sources during provisioning and restoration in elastic optical networks and compared with SBVTs employing standard lasers (ML-SBVT). The two operating modes of MW-SBVT and ML-SBVT were evaluated 1) NO SLICE 2) SLICE (when slice-ability is applied) for two network operation scenarios; provisioning and restoration. In the NO SLICE configuration, the MW source provided lower blocking probability than multiple lasers, both during provisioning and restoration. In the SLICE configuration, multiple lasers had a lower blocking probability during provisioning, whereas during restoration blocking depended on the network load. For low and medium network loads, the MW source performed better, while at high network load, multiple lasers had lower blocking probability. Another important observation was that during provisioning, the MW source had the same performance for both SLICE and NO SLICE configurations, while multiple lasers performance improved with the SLICE mode. It appears that during restoration, spectrum efficiency benefit provided by the MW source, overcomes the additional RSA constraint [270].

The authors extended the work by considering limited number of transponders in the node (previous study assumed that unlimited number of transponders is available in the node).
Network performance as a function of number of available transponders in each node and the offered network load was investigated in a dynamic scenario. In addition, a novel routing, spectrum and transponder assignment (RSTA) algorithm was proposed. It combined the transponder selection with RSA by taking into account the knowledge of transponder technology, current availability, and the constraints introduced by each specific transponder [77], [271]. The study evaluated the network performance for two different transponder technologies (ML-SBVT and MW SBVT), considering two traffic profiles (400 Gb/s traffic only and hybrid traffic) and three node architectures $(100 \% M W, 100 \% M L, x \% M W$ and $y \% M L)$, where $\mathrm{x}$ and $\mathrm{y}$ represent respectively the percentage of the MW and ML transmitters within the node. It was found that the blocking problem was resolved into two components: transponder blocking and spectrum blocking. The spectrum blocking relates to continuity constraint, contiguity constraint and spectrum scarcity, while transponder blocking refers to unavailability of transponders. It was observed that MW SBVT technology is better for superchannels due to improved SE and ML-SBVT is more suitable, when slice-ability is applied, owing to absence of any carrier spacing constraint. The performance of RSA and RSTA was also compared in a heterogeneous node architecture, where the node was equipped with both type of transponders in different percentages $(x \% M W$ and $y \% M L)$, to combine their respective benefits. The RSTA scheme provided significant network performance enhancement, compared to conventional RSA schemes, by efficient assignment of a transponder to a specific light-path request, depending on the transponder technology and the light-path spectrum requirements [77], [271].

Another, unanswered question is what would be the blocking probability, when terabit super-channels that need larger spectral contiguity were also included in mix traffic scenario, a most likely situation. The current OFC schemes provide symmetric spacing, which can be another source of blocking for future applications. OFC designs capable of providing both symmetric and arbitrary asymmetric carrier spacing will be an attractive solution. The findings of the work described above highlight the need for alternate solutions for carrier deployment, taking advantages of both types of sources.

\section{B. Optical carrier source deployment strategies}

As we described earlier, the reference architecture and above reports have mainly assumed a dedicated carrier source for each transponder. In such case unused carriers cannot be shared with other transponders as shown in the Fig. 29 (left). Alternative schemes for carrier source implementation in the node should also be explored to overcome this limitation. The notion is supported by the fact that a wide range of OFC generators providing few to hundreds of carriers have been reported in the literature. The dedicated SCG can accommodate only a few of these solutions. It is more likely that a single solution will not be suitable for all network applications and domains.

The idea of having two types of transponder technologies, combining the benefits of ML-SBVT and MW-SBVT [77] for enhanced network performance is interesting, but not without 
drawbacks. First, it is in contrast to the concept of having a bandwidth variable transponder supporting multiple rates and reach. Secondly, it will increase inventory cost because two types of spares have to be maintained. It is important to explore a carrier source deployment strategy that provides benefits of both MW and ML sources by overcoming the limitations of the former. Two centralized source deployment strategies have been reported so far: carrier source in the network (CSiNet) and carrier source in the node $(\mathrm{CSiN})$.

1) Carrier source in the network (CSiNet): The authors in [273] presented the design and evaluation of elastic optical network with a centralized multi-carrier light source (C-MCLS) [272] shown in the Fig. 29 (centre). This is an extension of their previous works for fixed grid networks [274], [275]. The idea is to have a centralized carrier source for all the nodes in the network. In this architecture, the elastic ROADM drops both the data signal and the carriers. However, the proposed architecture ignores the need for LO's for coherent detection in the receiver, which is essential in terabit flexible transponders. All the carriers are always available in the network and are dropped on demand. Since such sources provide hundreds of carriers, covering C-band, the comb bandwidth does not limit network performance. Similarly, unlike dedicated sources on each board, contiguity and limited carrier spacing constraint is not applicable, because any carrier can be dropped on demand by using a BV-WSS. However, concurrent tuning of subcarriers is not possible and reallocation of the same wavelength may also be restricted in some cases. The authors claim the solution to be cost and power efficient than distributed standard lasers.

2) Carrier source in the node $(C S i N)$ : Another possibility is to have a centralized flexible optical carrier source module (flex-OCSM) for the whole node as shown in the Fig. 29 (right). The authors in [276] proposed two different architectures of flex-OCSM modules, providing different level of flexibility and using different comb generation techniques. This deployment strategy is aimed at addressing the network performance issues of the dedicated comb SCGs and to decrease the cost and power consumption attributed to carriers in a node. The carriers in flex-OCSM can also be used as LO in the coherent receiver and, unlike C-MCLS, all carriers need not to be present on transmission fiber all the time. The results showed that, using centralized schemes, cost and power consumption can be reduced by an order of 4 . The overall contribution of the cost of the carrier generation subsystem to the cost of a SBVT can be decreased from $15 \%$ to $5 \%$ and power from $10 \%$ to $3 \%$ compared to III-V dedicated SCG.

\section{CONCLUSION}

From the survey and discussions presented in this paper, it can be concluded that comb based SCGs can be instrumental for dynamic networking in next generation optical networks. However, such carrier sources have to provide comparable optical performance, stability and significant cost savings if they are to replace standard lasers or III-V integrated lasers. Techniques providing few to tens and hundreds of lines have been demonstrated and there is no single "one fits all" solution. The choice of OFC and its ideal characteristics are dependent on the target application. The techniques providing tens of lines can be useful for modular designs, where SCGs subsystem is dedicated to a specific transponder module. The optical characteristics, transmission and network performance and TE feasibility of such sources has been evaluated and demonstrated.

Future work/ open issues: However, there are still several open issues which entail further research. The improved integrated designs (with reduced device imperfections) and advanced material systems (providing enhanced modulation index and power efficiency) can significantly improve stability and optical characteristics of high line count EO OFCs such as parametric and RFS OFCs. The network performance (in terms of blocking probability) of flex-OCSMs still needs to be evaluated. Likewise, innovative low cost flexible optical carrier de-multiplexing/filtering techniques with smaller foot print need to be explored. The techno-economic analysis need to be further extended by considering new flexible de-multiplexing technologies. The long term stability (reliability predictions) of both dedicated and centralized designs is yet to be estimated, to guarantee stable network operations. The number of required carriers in future SDM networks will certainly increase, paving the way to incorporate flexible sources in real networks, however, the requisite features may differ. It would be ideal to have different functional blocks of SCGs on a single photonic integrated circuit (PIC). Emerging OFC generation techniques based on micro-ring resonators (MRR) can be next choice because both carrier generation and flexible filtering functions can be obtained using MRRs. However, innovative techniques must be investigated to overcome the need for high power pump lasers. Nevertheless, the techno-economic viability of SCG highly dependent on the technological advancement in photonic and electronic integration such as $14 \mathrm{~nm}$ CMOS electronics and advanced silicon photonics.

\section{ACKNOWLEDGMENT}

This work was supported in part by the Science Foundation Ireland (SFI) Career Development Award -SFI 15/CDA/3640 and the Technology Innovation Development Award -SFI 16/TIDA/4011.

The authors would also like to thank Andrew Lord, BT Research UK and Deseada Gutierrez, Pilot Photonics, Ireland, for their useful insights and discussions.

\section{REFERENCES}

[1] J. L. Hall, "Optical frequency measurement: 40 years of technology revolutions," IEEE Journal of Selected Topics in Quantum Electronics, vol. 6, no. 6, pp. 1136-1144, Nov. 2000.

[2] T. W. Hansch, "Nobel lecture: Passion for precision*," Reviews of Modern Physics, vol. 78, pp. 1297-1309, Nov. 2006. [Online] Available: http://link.aps.org/doi/10.1103/RevModPhys.78.1297

[3] J. L. Hall, "Nobel lecture: Defining and measuring optical frequencies*," Reviews of Modern Physics, vol. 78, pp. 1279-1295, Nov. 2006. [Online]. Available: http://link.aps.org/doi/10.1103/RevModPhys.78.1279

[4] R. Holzwarth, T. Udem, T. W. Hänsch, J. C. Knight, W. J. Wadsworth, and P. S. J. Russell, "Optical frequency synthesizer for precision spectroscopy," Physical Review Letters, vol. 85, pp. 2264-2267, Sept. 2000. [Online]. Available: http://link.aps.org/doi/10.1103/PhysRevLett.85.2264 


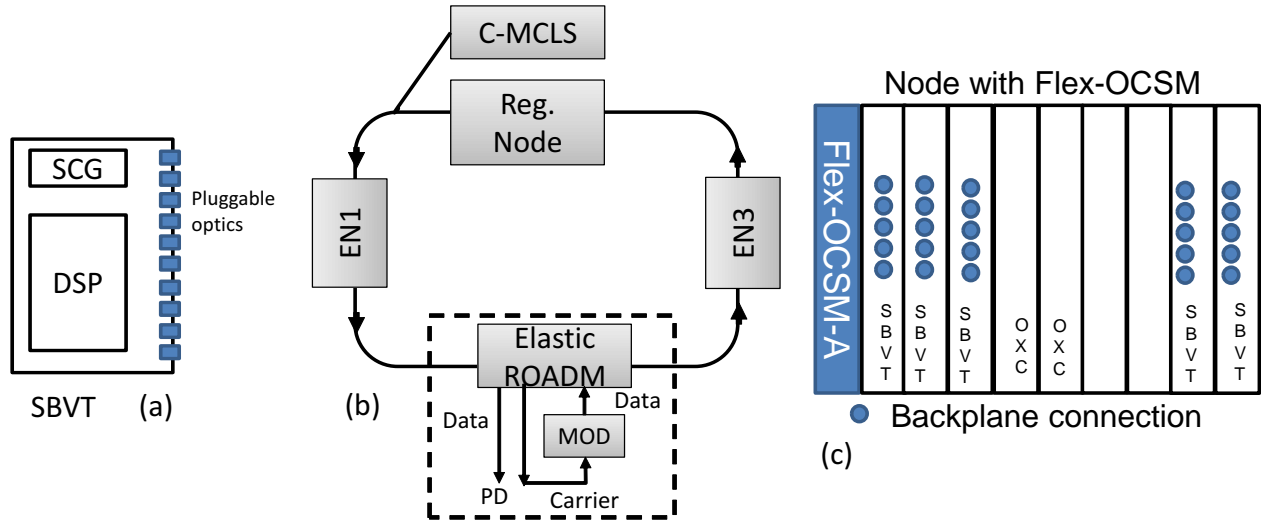

Figure 29: Carrier source deployment strategies: (a) Carrier source in board (CSiB) (b) Carrier source in network (CSiNet), EN1, edge node, MOD, modulator, PD, photodetector optical carriers generated by the CMCLS are dropped at the source nodes and used for uplink transmission. Data is added to the network after external modulations of the carriers and dropped at the destination nodes [272], (c) Proposed carrier source in node (CSiN).

[5] T. Steinmetz, T. Wilken, C. Araujo-Hauck, R. Holzwarth, T. W. Hänsch, L. Pasquini, A. Manescau, S. D'Odorico, M. T. Murphy, T. Kentischer, W. Schmidt, and T. Udem, "Laser frequency combs for astronomical observations," Science, vol. 321, no. 5894, pp. 1335-1337, 2008. [Online]. Available: http://science.sciencemag.org/content/321/5894/1335

[6] V. R. Supradeepa, C. M. Long, and A. M. Weiner, "Comb-based radio frequency photonic filters with rapid tunability and high selectivity," Nature Photonics, vol. 6, pp. 186-194, 2012.

[7] C. W. Chou, D. B. Hume, T. Rosenband, and D. J. Wineland, "Optical clocks and relativity," Science, vol. 329, no. 5999, pp. 1630-1633, 2010. [Online]. Available: http://science.sciencemag.org/content/329/5999/1630

[8] N. R. Newbury, "Searching for applications with a fine-tooth comb," Nature Photonics, vol. 5, pp. 186-188, 2011.

[9] K. Nosu, "Advanced coherent lightwave technologies," IEEE Communications Magazine, vol. 26, no. 2, pp. 15-21, Feb. 1988.

[10] V. Torres-Company and A. M. Weiner, "Optical frequency comb technology for ultra-broadband radio-frequency photonics," Laser \& Photonics Reviews, vol. 8, no. 3, pp. 368-393, 2014. [Online]. Available: http://dx.doi.org/10.1002/lpor.201300126

[11] S. A. Diddams, "The evolving optical frequency comb [invited]," Journal of the Optical Society of America B, vol. 27, no. 11, pp. B51-B62, Nov. 2010. [Online]. Available: http://josab.osa.org/abstract.cfm?URI=josab-27-11-B51

[12] P. Delfyett, I. Ozdur, N. Hoghooghi, M. Akbulut, J. Davila-Rodriguez, and S. Bhooplapur, "Advanced ultrafast technologies based on optical frequency combs," IEEE Journal of Selected Topics in Quantum Electronics, vol. 18, no. 1, pp. 258-274, Jan. 2012

[13] M. Fujiwara, M. Teshima, J. Kani, H. Suzuki, N. Takachio, and K. Iwatsuki, "Optical carrier supply module using flattened optical multicarrier generation based on sinusoidal amplitude and phase hybrid modulation," Journal of Lightwave Technology, vol. 21, no. 11, pp. 2705-2714, Nov. 2003.

[14] M. Jinno, H. Takara, B. Kozicki, Y. Tsukishima, Y. Sone, and S. Matsuoka, "Spectrum-efficient and scalable elastic optical path network: architecture, benefits, and enabling technologies," IEEE Communications Magazine, vol. 47, no. 11, pp. 66-73, Nov. 2009.

[15] O. Gerstel, M. Jinno, A. Lord, and S. J. B. Yoo, "Elastic optical networking: A new dawn for the optical layer?" IEEE Communications Magazine, vol. 50, no. 2, pp. s12-s20, Feb. 2012.

[16] G. Bosco, V. Curri, A. Carena, P. Poggiolini, and F. Forghieri, "On the performance of Nyquist-WDM Terabit Superchannels Based on PMBPSK, PM-QPSK, PM-8QAM or PM-16QAM Subcarriers," Journal of Lightwave Technology, vol. 29, no. 1, pp. 53-61, Jan. 2011.

[17] W. Shieh and C. Athaudage, "Coherent optical orthogonal frequency division multiplexing," Electronics Letters, vol. 42, no. 10, pp. 587589, May 2006.

[18] X. Ouyang, O. A. Dobre, and J. Zhao, "Unbiased Channel Estimation Based on the Discrete Fresnel Transform for CO-OFDM Systems,"
IEEE Photonics Technology Letters, vol. 29, no. 8, pp. 691-694, Apr 2017.

[19] M. Secondini, T. Foggi, F. Fresi, G. Meloni, F. Cavaliere, G. Colavolpe, E. Forestieri, L. Poti, R. Sabella, and G. Prati, "Optical TimeFrequency Packing: Principles, Design, Implementation, and Experimental Demonstration," Journal of Lightwave Technology, vol. 33 , no. 17 , pp. $3558-3570$, Sept. 2015.

[20] I. Tomkos, S. Azodolmolky, J. Pareta, D. Careglio, and E. Palkopoulou, "A tutorial on the flexible optical networking paradigm: State of the art, trends, and research challenges," Proceedings of the IEEE, vol. 102, no. 9, pp. 1317-1337, Sept. 2014

[21] A. S. Thyagaturu, A. Mercian, M. P. McGarry, M. Reisslein, and W. Kellerer, "Software Defined Optical Networks (SDONs): A Comprehensive Survey," IEEE Communications Surveys Tutorials, vol. 18, no. 4, pp. 2738-2786, 2016.

[22] Spectral Grids for WDM Applications: DWDM Frequency Grid, Recommendation G.694.1, International Telecommunication Union Telecommunication Standardization Sector (ITU-T), Feb. 2012., Std.

[23] Architecture of Optical Transport Networks, Recommendation G.872, International Telecommunication Union Telecommunication Standardization Sector (ITU-T), Oct. 2012., Std.

[24] N. Sambo, P. Castoldi, A. D'Errico, E. Riccardi, A. Pagano, M. S. Moreolo, J. M. Febrega, D. Rafique, A. Napoli, S. Frigerio, E. H. Salas, G. Zervas, M. Nolle, J. K. Fischer, A. Lord, and F. P. Gimnez, "Next generation sliceable bandwidth variable transponders," IEEE Communications Magazine, vol. 53, no. 2, pp. 163-171, Feb. 2015.

[25] N. Sambo, A. D’Errico, C. Porzi, V. Vercesi, M. Imran, F. Cugini, A. Bogoni, L. Poti, and P. Castoldi, "Sliceable transponder architecture including multiwavelength source," IEEE/OSA Journal of Optical Communications and Networking, vol. 6, no. 7, pp. 590-600, July 2014

[26] O. Omomukuyo, D. Chang, O. Dobre, R. Venkatesan, and T. M. N. Ngatched, "Robust Frame and Frequency Synchronization Based on Alamouti Coding for RGI-CO-OFDM," IEEE Photonics Technology Letters, vol. 28, no. 24, pp. 2783-2786, Dec. 2016.

[27] C. R. Doerr, "Silicon photonic integration in telecommunications," Frontiers in Physics, vol. 3, no. 37, Aug. 2015.

[28] G. Bosco, A. Carena, V. Curri, P. Poggiolini, and F. Forghieri, "Performance Limits of Nyquist-WDM and CO-OFDM in High-Speed PMQPSK Systems," IEEE Photonics Technology Letters, vol. 22, no. 15, pp. 1129-1131, Aug. 2010.

[29] G. Meloni, T. Rahman, and L. Poti, "Experimental Comparison of Transmission Performance for Nyquist WDM and Time Frequency Packing," Journal of Lightwave Technology, vol. 33, no. 24, pp. 52615268, Dec. 2015.

[30] X. Liu and S. Chandrasekhar, "Superchannel for next-generation optical networks," in Optical Fiber Communications Conference and Exhibition (OFC), Mar. 2014, pp. 1-33.

[31] R. Schmogrow, R. Bouziane, M. Meyer, P. A. Milder, P. C. Schindler, P. Bayvel, R. I. Killey, W. Freude, and J. Leuthold, "Real-time digital Nyquist-WDM and OFDM signal generation: Spectral efficiency versus 
DSP complexity," in 38th European Conference and Exhibition on Optical Communications, Sept. 2012, pp. 1-3.

[32] S. Chandrasekhar and X. Liu, "OFDM Based Superchannel Transmission Technology," Journal of Lightwave Technology, vol. 30, no. 24, pp. 3816-3823, Dec. 2012.

[33] O. Omomukuyo, D. Chang, J. Zhu, O. Dobre, R. Venkatesan, T. Ngatched, and C. Rumbolt, "Joint timing and frequency synchronization based on weighted CAZAC sequences for reduced-guard-interval CO-OFDM systems," Optics Express, vol. 23, no. 5, pp. 5777-5788, Mar. 2015.

[34] S. Zhang, D. Chang, O. A. Dobre, O. Omomukuyo, X. Lin, and R. Venkatesan, "Training Symbol-Based Equalization for Quadrature Duobinary PDM-FTN Systems," IEEE Photonics Technology Letters, vol. 29, no. 5, pp. 454-457, Mar. 2017.

[35] N. Sambo, G. Meloni, F. Paolucci, F. Cugini, M. Secondini, F. Fresi, L. Poti, and P. Castoldi, "Programmable Transponder, Code and Differentiated Filter Configuration in Elastic Optical Networks," Journal of Lightwave Technology, vol. 32, no. 11, pp. 2079-2086, June 2014.

[36] D. Chang, O. Omomukuyo, X. Lin, S. Zhang, O. A. Dobre, and R. Venkatesan, "Robust Faster-Than-Nyquist PDM-mQAM Systems With Tomlinson Harashima Precoding," IEEE Photonics Technology Letters, vol. 28, no. 19, pp. 2106-2109, Oct. 2016.

[37] L. Poti, G. Meloni, G. Berrettini, F. Fresi, M. Secondini, T. Foggi, G. Colavolpe, E. Forestieri, A. D'Errico, F. Cavaliere, R. Sabella, and G. Prati, "Casting $1 \mathrm{~Tb} / \mathrm{s}$ DP-QPSK communication into $200 \mathrm{GHz}$ bandwidth," in 38th European Conference and Exhibition on Optical Communications, Sept. 2012, pp. 1-3.

[38] M. S. Moreolo, J. M. Fabrega, L. Nadal, F. J. Vílchez, A. Mayoral, R. Vilalta, R. Muñoz, R. Casellas, R. Martínez, M. Nishihara, T. Tanaka, T. Takahara, J. C. Rasmussen, C. Kottke, M. Schlosser, R. Freund, F. Meng, S. Yan, G. Zervas, D. Simeonidou, Y. Yoshida, and K. I. Kitayama, "SDN-Enabled Sliceable BVT Based on Multicarrier Technology for Multiflow Rate, Distance and Grid Adaptation," Journal of Lightwave Technology, vol. 34, no. 6, pp. 1516-1522, March 2016.

[39] N. Sambo, G. Meloni, F. Paolucci, M. Imran, F. Fresi, F. Cugini, P. Castoldi, and L. Poti, "First demonstration of SDN-controlled SBVT based on multi-wavelength source with programmable and asymmetric channel spacing," in European Conference on Optical Communication (ECOC), Sept. 2014, pp. 1-3.

[40] Y. Wang, Z. McNulty, and H. Nguyen, "Network virtualization in spectrum sliced elastic optical path networks," Journal of Lightwave Technology, vol. 35, no. 10, pp. 1962-1970, May 2017.

[41] Y. Ou, A. Hammad, S. Peng, R. Nejabati, and D. Simeonidou, "Online and offline virtualization of optical transceiver," IEEE/OSA Journal of Optical Communications and Networking, vol. 7, no. 8, pp. 748-760, Aug. 2015.

[42] Y. Ou, S. Yan, A. Hammad, B. Guo, S. Peng, R. Nejabati, and D. Simeonidou, "Demonstration of Virtualizeable and Software-Defined Optical Transceiver," Journal of Lightwave Technology, vol. 34, no. 8, pp. 1916-1924, Apr. 2016.

[43] A. Blenk, A. Basta, J. Zerwas, M. Reisslein, and W. Kellerer, "Control Plane Latency With SDN Network Hypervisors: The Cost of Virtualization," IEEE Transactions on Network and Service Management, vol. 13, no. 3, pp. 366-380, Sept. 2016.

[44] Y. Ou, F. Meng, P. M. Anadarajah, S. Yan, A. Aguado, M. D. G. Pascual, R. Nejabati, and D. Simeonidou, "Investigation of optical impacts on virtualization using SDN-enabled transceiver and optical monitoring," in Optical Fiber Communications Conference and Exhibition (OFC), Mar. 2017, pp. 1-3.

[45] J. Schonwalder, M. Bjorklund, and P. Shafer, "Network configuration management using NETCONF and YANG," IEEE Communications Magazine, vol. 48, no. 9, pp. 166-173, Sept. 2010.

[46] M. Bjorklund, "YANG - A Data Modeling Language for the Network Configuration Protocol (NETCONF)," IETF RFC 6020, Tech. Rep., Oct. 2010.

[47] A. Shaikh, T. Hofmeister, V. Dangui, and V. Vusirikala, "Vendorneutral network representations for transport SDN," in Optical Fiber Communications Conference and Exhibition (OFC), Mar. 2016, pp. $1-3$.

[48] [Online]. Available: http://www.openconfig.net/

[49] J. L. de Vergara, V. Lopez, D. King, Y. Lee, and Z. Ali, "YANG data model for Flexi-Grid Optical Networks," IETF, Tech. Rep., Jan. 2017.

[50] M. Dallaglio, N. Sambo, F. Cugini, and P. Castoldi, "YANG Models for Vendor-Neutral Optical Networks, Reconfigurable through State Machine," IEEE Communications Magazine, vol. 55, no. 8, pp. 170178, Aug. 2017.
[51] N. Sambo, P. Giardina, I. Sartzetakis, A. Sgambelluri, F. Fresi, M. Dallaglio, G. Meloni, G. Bernini, K. C. amd P. Castoldi, and E. Varvarigos, "Experimental Demonstration of Network Automation Based on QoT Estimation and Monitoring in Both Single- and Multi-Domains," in 43rd European Conference and Exhibition on Optical Communication (ECOC), Sept. 2017, pp. 1-3.

[52] F. Cugini, F. Paolucci, G. Meloni, G. Berrettini, M. Secondini, F. Fresi, N. Sambo, L. Poti, and P. Castoldi, "Push-Pull Defragmentation Without Traffic Disruption in Flexible Grid Optical Networks," Journal of Lightwave Technology, vol. 31, no. 1, pp. 125-133, Jan. 2013.

[53] F. Paolucci, A. Castro, F. Fresi, M. Imran, A. Giorgetti, B. Bhownik, G. Berrettini, G. Meloni, F. Cugini, L. Velasco, L. Poti, and P. Castoldi, "Active PCE demonstration performing elastic operations and hitless defragmentation in flexible grid optical networks," Photonic Network Communications, vol. 29, no. 1, pp. 57-66, Feb. 2015. [Online]. Available: http://dx.doi.org/10.1007/s11107-014-04640

[54] Z. Haiyi, Z. Wenyu, and W. Bingbing, "Considerations on transport networks technologies evolution," China Communications, vol. 10, no. 4, pp. 7-18, Apr. 2013.

[55] http://www.oiforum.com/wp-content/uploads/OIF-ITLA-MSA-01.3.pdf, Optical Internetworking Forum Std.

[56] P. Fernando, R. Ramos, and A. Seeds, "Optical carrier synthesis in coherent lightwave systems," in IEE Colloquium on Sources for Coherent Optical Communication, Jan. 1991, pp. 1-7.

[57] R. Drever, J. Hall, F. Kowalski, J. Hough, G. Ford, A. Munley, and $\mathrm{H}$. Ward, "Laser phase and frequency stabilization using an optical resonator," Applied Physics B, vol. 31, no. 2, pp. 97-105, Feb. 1983.

[58] J. Kim, B. S. Choi, H. Yun, S. H. Oh, J.-H. Lee, H. Ko, K.-S Choi, S. Park, J.-T. Moon, and M.-H. Park, "Thermally controlled wavelength locker integrated in widely tunable SGDBR-LD module," IEEE Photonics Technology Letters, vol. 16, no. 11, pp. 2430-2432, Nov 2004.

[59] Y. Yokoyama, H. Hatakeyama, K. Naniwae, K. Satoh, and M. Yamaguchi, "94-channel wavelength-selectable light source module with integrated multiwavelength locker," in Optical Fiber Communication Conference and Exhibit, Mar. 2002, pp. 206-208.

[60] B. Collings, "New devices enabling software-defined optical networks," IEEE Communications Magazine, vol. 51, no. 3, pp. 66-71, Mar. 2013.

[61] T. Koike-Akino, D. S. Millar, K. Kojima, and K. Parsons, "Laser frequency drift compensation with Han-Kobayashi coding in superchannel nonlinear optical communications," in European Conference on Optical Communication (ECOC), Sept. 2015, pp. 1-3.

[62] A. Saeki and K. Kato, "Fine wavelength stabilization of the DFB laser at the flexible grid by photo-mixing with reference wavelengths," in Asia-Pacific Microwave Conference (APMC), Nov. 2014, pp. 12851287.

[63] P. Delfyett, S. Gee, M.-T. Choi, H. Izadpanah, W. Lee, S. Ozharar, F. Quinlan, and T. Yilmaz, "Optical frequency combs from semiconductor lasers and applications in ultrawideband signal processing and communications," Journal of Lightwave Technology, vol. 24, no. 7, pp. 2701-2719, July 2006.

[64] S. Gringeri, N. Bitar, and T. Xia, "Extending software defined network principles to include optical transport," IEEE Communications Magazine, vol. 51, no. 3, pp. 32-40, Mar. 2013.

[65] M. C. Larson, "Widely tunable semiconductor lasers," in Optical Fiber Communications Conference and Exhibition (OFC), Mar. 2014, pp. $1-25$.

[66] A. Castro, F. Paolucci, F. Fresi, M. Imran, B. Bhowmik, G. Berrettini, G. Meloni, A. Giorgetti, F. Cugini, L. Velasco, L. Poti, and P. Castoldi, "Experimental demonstration of an active stateful PCE performing elastic operations and hitless defragmentation," in 39th European Conference and Exhibition on Optical Communication (ECOC), Sept. 2013, pp. 1-3.

[67] V. Vercesi, M. Scaffardi, and A. Bogoni, "Hitless multiwavelength source reconfiguration for flexible optical networks," in International Conference on Optical Network Design and Modeling (ONDM), May 2015, pp. 145-150.

[68] B.-P. Kuo, E. Myslivets, V. Ataie, E. Temprana, N. Alic, and S. Radic, "Wideband parametric frequency comb as coherent optical carrier," Journal of Lightwave Technology, vol. 31, no. 21, pp. 3414-3419, Nov. 2013.

[69] Wu, "Carrier recovery in a coherent optical receiver," US Patent US 7606498 B1, 2009.

[70] A. D'Errico and G. Contestabile, "Next generation terabit transponder," in Optical Fiber Communication Conference. Optical Society of America, 2016, p. W4B.4. 
[71] X. Yi, N. Fontaine, R. Scott, and S. Yoo, "Tb/s Coherent Optical OFDM Systems Enabled by Optical Frequency Combs," Journal of Lightwave Technology, vol. 28, no. 14, pp. 2054-2061, July 2010.

[72] S. Chandrasekhar, X. Liu, B. Zhu, and D. Peckham, "Transmission of a $1.2-\mathrm{Tb} / \mathrm{s}$ 24-carrier no-guard-interval coherent OFDM superchannel over $7200-\mathrm{km}$ of ultra-large-area fiber," in 35th European Conference on Optical Communication, ECOC '09., vol. 2009-Supplement, Sept. 2009, pp. 1-2.

[73] T. J. Xia, G. A. Wellbrock, Y. K. Huang, M. F. Huang, E. Ip, P. N. Ji, D. Qian, A. Tanaka, Y. Shao, T. Wang, Y. Aono, and T. Tajima, "21.7 Tb/s field trial with 22 DP-8QAM/QPSK optical superchannels over 1,503-km of installed SSMF," in Optical Fiber Communication Conference and Exposition and the National Fiber Optic Engineers Conference (OFC/NFOEC), Mar. 2012, pp. 1-3.

[74] T. Sakamoto, G.-W. Lu, and T. Kawanishi, "Filter-less multi-tone coherent orthogonal detection for multi-channel reception of superchannel/OFDM signals," in Optical Fiber Communication Conference and Exposition and the National Fiber Optic Engineers Conference (OFC/NFOEC), Mar. 2013, pp. 1-3.

[75] M. A. Soto, M. Alem, M. A. Shoaie, A. Vedadi, C.-S. Brès, and L. T. T. Schneider, "Optical sinc-shaped Nyquist pulses of exceptional quality," Nature Communications, vol. 4, no. 2898(2013), Dec. 2013. [Online]. Available: http://dx.doi.org/10.1038/ncomms3898

[76] J. Zhang, J. Yu, Y. Fang, and N. Chi, "High Speed All Optical Nyquist Signal Generation and Full-band Coherent Detection," Scientific Reports, vol. 4, no. 6156, Aug. 2014. [Online]. Available: http://dx.doi.org/10.1038/srep06156

[77] M. Dallaglio, A. Giorgetti, N. Sambo, L. Velasco, and P. Castoldi, "Routing, Spectrum, and Transponder Assignment in Elastic Optical Networks," Journal of Lightwave Technology, vol. 33, no. 22, pp. 4648-4658, Nov. 2015.

[78] R. Slavik, S. Farwell, M. Wale, and D. Richardson, "Compact Optical Comb Generator Using InP Tunable Laser and Push-Pull Modulator," IEEE Photonics Technology Letters, vol. 27, no. 2, pp. 217-220, Jan. 2015.

[79] C. Weimann, P. C. Schindler, R. Palmer, S. Wolf, D. Bekele, D. Korn, J. Pfeifle, S. Koeber, R. Schmogrow, L. Alloatti, D. Elder, H. Yu, W. Bogaerts, L. R. Dalton, W. Freude, J. Leuthold, and C. Koos, "Silicon-organic hybrid (SOH) frequency comb sources for terabit/s data transmission," Optics Express, vol. 22, no. 3, pp. 3629-3637, Feb. 2014.

[80] J. Macario, P. Yao, S. Shi, A. Zablocki, C. Harrity, R. D. Martin, C. A. Schuetz, and D. W. Prather, "Full spectrum millimeter-wave modulation," Optics Express, vol. 20, no. 21, pp. $23623-23629$, Oct. 2012. [Online]. Available: http://www.opticsexpress.org/abstract.cfm?URI=oe-20-21-23623

[81] D. Chen, H. R. Fetterman, A. Chen, W. H. Steier, L. R. Dalton, W. Wang, and Y. Shi, "Demonstration of $110 \mathrm{GHz}$ electro-optic polymer modulators," Applied Physics Letters, vol. 70, no. 25, pp. 3335-3337, June 1997.

[82] L. Alloatti, R. Palmer, S. Diebold, K. P. Pahl, B. Chen, R. Dinu, M. Fournier, J. Fedeli, T. Zwick, W. Freude, C. Koos, and J. Leuthold, "100 GHz silicon-organic hybrid modulator," Light: Science \& Applications, vol. 3, p. e173, May 2014.

[83] T. Sakamoto, T. Kawanishi, and M. Izutsu, "Asymptotic formalism for ultraflat optical frequency comb generation using a Mach-Zehnder modulator," Optics Letters, vol. 32, no. 11, pp. 1515-1517, June 2007.

[84] M. Fujiwara, J. Kani, H. Suzuki, K. Araya, and M. Teshima, "Flattened optical multicarrier generation of $12.5 \mathrm{GHz}$ spaced 256 channels based on sinusoidal amplitude and phase hybrid modulation," Electronics Letters, vol. 37, no. 15, pp. 967-968, July 2001.

[85] Y. Tanaka, Y. Matsunaga, T. Hoshi, T. Shioda, and T. Kurokawa, "Multicarrier Light Source with $50 \mathrm{GHz}$ Spacing and Its Application in Dense Wavelength Division Multiplexing System," Japanese Journal of Applied Physics, vol. 48, no. 9S2, p. 09LF04, Sept. 2009. [Online]. Available: http://stacks.iop.org/1347-4065/48/i=9S2/a=09LF04

[86] V. Torres-Company, J. Lancis, and P. Andrés, "Lossless equalization of frequency combs," Optics Letters, vol. 33, no. 16, pp. 1822-1824, Aug. 2008

[87] R. Wu, V. R. Supradeepa, C. M. Long, D. E. Leaird, and A. M. Weiner, "Generation of very flat optical frequency combs from continuous-wave lasers using cascaded intensity and phase modulators driven by tailored radio frequency waveforms," Optics Letters, vol. 35, no. 19, pp. 3234-3236, Oct. 2010. [Online]. Available: http://ol.osa.org/abstract.cfm?URI=ol-35-19-3234

[88] A. Metcalf, V. Torres-Company, D. Leaird, and A. Weiner, "HighPower Broadly Tunable Electrooptic Frequency Comb Generator,"
IEEE Journal of Selected Topics in Quantum Electronics, vol. 19, no. 6 , pp. 231-236, Nov. 2013.

[89] H. Suzuki, M. Fujiwara, N. Takachio, K. Iwatsuki, T. Kitoh, and T. Shibata, " $12.5 \mathrm{ghz}$ spaced $1.28 \mathrm{tb} / \mathrm{s}$ (512-channel x $2.5 \mathrm{gb} / \mathrm{s})$ superdense wdm transmission over $320 \mathrm{~km} \mathrm{smf} \mathrm{using} \mathrm{multiwavelength}$ generation technique," Photonics Technology Letters, IEEE, vol. 14, no. 3, pp. 405-407, March 2002.

[90] H. Suzuki, M. Fujiwara, and K. Iwatsuki, "Application of superDWDM technologies to terrestrial terabit transmission systems," Journal of Lightwave Technology, vol. 24, no. 5, pp. 1998-2005, May 2006.

[91] W. Mao, P. Andrekson, and J. Toulouse, "Investigation of a spectrally flat multi-wavelength DWDM source based on optical phase- and intensity-modulation," in Optical Fiber Communication Conference (OFC), vol. 1, Feb. 2004, pp. 235-236.

[92] M. Doi, M. Sugiyama, K. Tanaka, and M. Kawai, "Advanced LiNbO3 optical modulators for broadband optical communications," IEEE Journal of Selected Topics in Quantum Electronics, vol. 12, no. 4, pp. 745-750, July 2006.

[93] Y. Dou, H. Zhang, and M. Yao, "Improvement of flatness of optical frequency comb based on nonlinear effect of intensity modulator," Optics Letters, vol. 36, no. 14, pp. 2749-2751, July 2011. [Online]. Available: http://ol.osa.org/abstract.cfm?URI=ol-36-14-2749

[94] R. Wei, J. Yan, Y. Peng, X. Yao, M. Bai, and Z. Zheng, "Optical frequency comb generation based on electro-optical modulation with high-order harmonic of a sine RF signal," Optics Communications, vol. 291, pp. 269-273, Mar. 2013. [Online]. Available: http://www.sciencedirect.com/science/article/pii/S0030401812012655

[95] R. Jales Lima Ferreira, M. de Lacerda Rocha, E. Da Silva, and D. Moutinho Pataca, "Cascade of amplitude- and phasemodulator in four different configurations of superchannel generation," in SBMO/IEEE MTT-S International Microwave Optoelectronics Conference (IMOC), Aug. 2013, pp. 1-5.

[96] J. Veselka and S. Korotky, "A multiwavelength source having precise channel spacing based on external modulation and fiber Kerr nonlinearity," in Conference on Optical Fiber Communication (OFC), Feb. 1997, pp. 299-301.

[97] _ - "A multiwavelength source having precise channel spacing for WDM systems," IEEE Photonics Technology Letters, vol. 10, no. 7, pp. 958-960, July 1998 .

[98] T. Sakamoto, T. Kawanishi, and M. Izutsu, "Optimization of ElectroOptic Comb Generation Using Conventional Mach-Zehnder Modulator," in IEEE International Topical Meeting on Microwave Photonics, Oct. 2007, pp. 50-53.

[99] A. Mishra and I. Tomkos, "A novel cost-effective combline generation and cross-talk mitigation in optical OFDM signal using optical iFFT circuits," in 18th International Conference on Telecommunications (ICT), May 2011, pp. 107-112.

[100] A. Mishra, R. Schmogrow, I. Tomkos, D. Hillerkuss, C. Koos, W. Freude, and J. Leuthold, "Flexible RF-Based Comb Generator," IEEE Photonics Technology Letters, vol. 25, no. 7, pp. 701-704, Apr. 2013.

[101] T. Saikai, T. Yamamoto, H. Yasaka, and E. Yamada, "Flat-top optical frequency comb block generation using InP-based Mach-Zehnder modulator," in International Conference on Indium Phosphide and Related Materials (IPRM), May 2013, pp. 1-2.

[102] T. Yamamoto, K. Hitomi, W. Kobayashi, and H. Yasaka, "Optical Frequency Comb Block Generation by Using Semiconductor MachZehnder Modulator,' IEEE Photonics Technology Letters, vol. 25, no. 1, pp. 40-42, Jan. 2013.

[103] A. Hraghi, M. Menif, and S. Ben Abid, "Optimization of Optical Flat Comb Source based on Dual-Arm Mach-Zehnder modulator for flexgrid terabit superchannel WDM-Nyquist systems," in 16th International Conference on Transparent Optical Networks (ICTON), July 2014, pp. $1-4$.

[104] J. Botia, H. Yepes, A. Cardenas, and G. Quintero, "Fuzzy entropies by adequacy and non-adequacy applied to the analysis of combs spectra stability," in IEEE International Conference on Computational Intelligence and Virtual Environments for Measurement Systems and Applications (CIVEMSA), July 2013, pp. 174-179.

[105] L. Shang, A. Wen, G. Lin, and Y. Gao, "A flat and broadband optical frequency comb with tunable bandwidth and frequency spacing," Optics Communications, vol. 331, pp. 262-266, Nov. 2014. [Online]. Available: http://www.sciencedirect.com/science/article/pii/S0030401814005768 
[106] A. D. Ellis and F. Gunning, "Spectral density enhancement using coherent WDM," IEEE Photonics Technology Letters, vol. 17, no. 2, pp. 504-506, Feb. 2005

[107] W. Li and J. Yao, "Optical frequency comb generation based on repeated frequency shifting using two Mach-Zehnder modulators and an asymmetric Mach-Zehnder interferometer," Optics Express, vol. 17, no. 26, pp. 23712-23718, Dec. 2009.

[108] K. Yonenaga, F. Inuzuka, S. Yamamoto, H. Takara, B. Kozicki, T. Yoshimatsu, A. Takada, and M. Jinno, "Bit-rate-flexible alloptical OFDM transceiver using variable multi-carrier source and DQPSK/DPSK mixed multiplexing," in Conference on Optical Fiber Communication - incudes post deadline papers (OFC), Mar. 2009, pp. $1-3$.

[109] S. Preussler, N. Wenzel, and T. Schneider, "Flat, rectangular frequency comb generation with tunable bandwidth and frequency spacing," Optics Letters, vol. 39, no. 6, pp. 1637-1640, Mar. 2014. [Online]. Available: http://ol.osa.org/abstract.cfm?URI=ol-39-6-1637

[110] M. Imran, F. Fresi, G. Meloni, and L. Poti, " $12.5 \mathrm{GHz}-100 \mathrm{GHz}$ tunable spacing optical carrier source for flexgrid bandwidth variable transponders," in International Conference on Optical Network Design and Modeling (ONDM), May 2015, pp. 157-161.

[111] C. Yuhua, C. Yang, L. Xianghua, Z. Zhou, and D. Zhu, "Generation of Optical Frequency Comb With Double Spectral Spacing Based on Two Cascaded Mach-Zehnder Modulators," in International Photonics and OptoElectronics Meetings. Optical Society of America, 2014, p. FTh4E.4.

[112] J. K. Hmood, S. D. Emami, K. A. Noordin, H. Ahmad, S. W. Harun, and H. M. Shalaby, "Optical frequency comb generation based on chirping of MachZehnder Modulators," Optics Communications, vol. 344, pp. 139-146, June 2015. [Online]. Available: http://www.sciencedirect.com/science/article/pii/S003040181500067X

[113] X. Zhou, X. Zheng, H. Wen, H. Zhang, and B. Zhou, "Generation of broadband optical frequency comb with rectangular envelope using cascaded intensity and dual-parallel modulators," Optics Communications, vol. 313, pp. 356-359, Feb. 2014. [Online]. Available: http://www.sciencedirect.com/science/article/pii/S0030401813009826

[114] S. Preussler, N. Wenzel, and T. Schneider, "Flexible Nyquist Pulse Sequence Generation with Variable Bandwidth and Repetition Rate," IEEE Photonics Journal, vol. 6, no. 4, pp. 1-8, Aug. 2014

[115] S. Ozharar, F. Quinlan, I. Ozdur, S. Gee, and P. Delfyett, "Ultraflat optical comb generation by phase-only modulation of continuous-wave light," IEEE Photonics Technology Letters, vol. 20, no. 1, pp. 36-38, Jan. 2008 .

[116] M. Yamamoto, Y. Tanaka, T. Shioda, T. Kurokawa, and K. Higuma, "Optical frequency comb generation using dual frequency optical phase modulation," in Integrated Photonics Research and Applications/Nanophotonics for Information Systems. Optical Society of America, Apr. 2005, p. ITuF5. [Online]. Available: http://www.osapublishing.org/abstract.cfm?URI=IPRA-2005-ITuF5

[117] Y. Yamamoto, T. Komukai, K. Suzuki, and A. Takada, "Multicarrier light source with flattened spectrum using phase modulators and dispersion medium," Journal of Lightwave Technology, vol. 27, no. 19, pp. $4297-4305$, Oct. 2009

[118] J. Zhang, J. Yu, L. Tao, Y. Fang, Y. Wang, Y. Shao, and N. Chi, "Generation of coherent and frequency-lock optical subcarriers by cascading phase modulators driven by sinusoidal sources," Journal of Lightwave Technology, vol. 30, no. 24, pp. 3911-3917, Dec. 2012.

[119] J. Zhang, J. Yu, Z. Dong, N. Chi, and X. Li, "Flattened optical comb generation using only phase modulators driven by single fundamental frequency sinusoidal sources with small frequency offset," in Optical Fiber Communication Conference and Exposition and the National Fiber Optic Engineers Conference (OFC/NFOEC), Mar. 2013, pp. 1-3.

[120] Y.-K. Huang, M.-F. Huang, E. Ip, E. Mateo, P. Ji, D. Qian, A. Tanaka, Y. Shao, T. Wang, Y. Aono, T. Tajima, T. Xia, and G. Wellbrock, "HighCapacity Fiber Field Trial Using Terabit/s All-Optical OFDM Superchannels With DP-QPSK and DP-8QAM/DP-QPSK Modulation," Journal of Lightwave Technology, vol. 31, no. 4, pp. 546-553, Feb. 2013.

[121] M.-F. Huang, A. Tanaka, E. Ip, Y.-K. Huang, D. Qian, Y. Zhang, S. Zhang, P. Ji, I. Djordjevic, T. Wang, Y. Aono, S. Murakami, T. Tajima, T. Xia, and G. Wellbrock, "Terabit/s Nyquist Superchannels in High Capacity Fiber Field Trials Using DP-16QAM and DP-8QAM Modulation Formats," Journal of Lightwave Technology, vol. 32, no. 4, pp. 776-782, Feb. 2014

[122] 1. Gheorma and G. Gopalakrishnan, "Flat frequency comb generation with an integrated dual-parallel modulator," IEEE Photonics Technology Letters, vol. 19, no. 13, pp. 1011-1013, July 2007.
[123] S. Zhang, J. Yan, Z. Xia, X. Yao, M. Bai, and Z. Zheng, "Generation of tunable optical frequency combs with a high side mode suppression ratio," in Conference on Lasers and Electro-Optics (CLEO), June 2014, pp. $1-2$.

[124] Q. Wang, L. Huo, Y. Xing, and B. Zhou, "Ultra-flat optical frequency comb generator using a single-driven dual-parallel Mach-Zehnder modulator," Optics Letters, vol. 39, no. 10, pp. 3050-3053, May 2014 [Online]. Available: http://ol.osa.org/abstract.cfm?URI=ol-39-10-3050

[125] M. Imran, F. Fresi, G. Meloni, B. Bhowmik, N. Sambo, and L. Poti, "A programmable optical few wavelength source for flexgrid optical networks," Optical Fiber Technology, vol. 30, pp. 100-111, July 2016. [Online]. Available: http://www.sciencedirect.com/science/article/pii/S1068520016300128

[126] C. O'Riordan, S. Fabbri, and A. D. Ellis, "Variable optical frequency comb source using a dual parallel Mach-Zehnder modulator," in 13th International Conference on Transparent Optical Networks (ICTON), June 2011, pp. 1-4.

[127] J. Zang, J. Wu, Y. Li, X. Nie, J. Qiu, and J. Lin, "Generation of Nyquist pulses using a dual parallel Mach-Zehnder modulator," in Conference on Lasers and Electro-Optics (CLEO), June 2014, pp. 1-2.

[128] M. Izutsu, S. Shikama, and T. Sueta, "Integrated optical SSB modulator/frequency shifter," IEEE Journal of Quantum Electronics, vol. 17, no. 11, pp. 2225-2227, Nov. 1981.

[129] T. Kawanishi and M. Izutsu, "Linear single-sideband modulation for high-SNR wavelength conversion," IEEE Photonics Technology Letters, vol. 16, no. 6, pp. 1534-1536, June 2004.

[130] S. Shimotsu, S. Oikawa, T. Saitou, N. Mitsugi, K. Kubodera, T. Kawanishi, and M. Izutsu, "Single side-band modulation performance of a LiNbO3 integrated modulator consisting of four-phase modulator waveguides," IEEE Photonics Technology Letters, vol. 13, no. 4, pp. 364-366, Apr. 2001

[131] S. Fabbri, C. O'Riordan, S. Sygletos, and A. Ellis, "Active stabilisation of single drive dual-parallel Mach-Zehnder modulator for single sideband signal generation," Electronics Letters, vol. 49, no. 2, pp. 135-136, Jan. 2013.

[132] Y. Ogiso, Y. Tsuchiya, S. Shinada, S. Nakajima, T. Kawanishi, and H. Nakajima, "High extinction-ratio (ER) Mach-Zehnder modulator (MZM) with an active Y-branch," IEEE Photonics Technology Letters, no. 12, pp. 941-943, June 2010.

[133] M. Lauermann, C. Weimann, A. Knopf, D. L. Elder, W. Heni, R. Palmer, D. Korn, P. Schindler, S. Koeber, L. Alloatti, H. Yu, W. Bogaerts, L. R. Dalton, C. Rembe, J. Leuthold, W. Freude, and C. Koos, "Integrated Silicon-Organic Hybrid ( $\mathrm{SOH}$ ) Frequency Shifter," in Optical Fiber Communication Conference. Optical Society of America, Mar. 2014, p. Tu2A.1.

[134] J. D. Bull, N. A. Jaeger, H. Kato, M. Fairburn, A. Reid, and P. Ghanipour, "40-GHz electro-optic polarization modulator for fiber optic communications systems," Proceedings of SPIE, vol. 5577, Dec. 2004

[135] C. Chen, F. Zhang, and S. Pan, "Generation of seven-line optical frequency comb based on a single polarization modulator," IEEE Photonics Technology Letters, vol. 25, no. 22, pp. 2164-2166, Nov. 2013.

[136] S. Pan and J. Yao, "A frequency-doubling optoelectronic oscillator using a polarization modulator," IEEE Photonics Technology Letters, vol. 21, no. 13, pp. 929-931, July 2009.

[137] C. He, S. Pan, R. Guo, Y. Zhao, and M. Pan, "Ultraflat optical frequency comb generated based on cascaded polarization modulators," Optics Letters, vol. 37, no. 18, pp. 3834-3836, Sept. 2012. [Online]. Available: http://ol.osa.org/abstract.cfm?URI=ol-37-18-3834

[138] T. Healy, F. C. G. Gunning, A. D. Ellis, and J. D. Bull, "Multiwavelength source using low drive-voltage amplitude modulators for optical communications," Optics Express, vol. 15, no. 6, pp. 29812986, Mar. 2007.

[139] C. Chen, C. He, D. Zhu, R. Guo, F. Zhang, and S. Pan, "Generation of a flat optical frequency comb based on a cascaded polarization modulator and phase modulator," Optics Letters, vol. 38, no. 16, pp. 3137-3139, Aug. 2013. [Online]. Available: http://ol.osa.org/abstract.cfm?URI=ol38-16-3137

[140] M. Wang and J. Yao, "Tunable optical frequency comb generation based on an optoelectronic oscillator," IEEE Photonics Technology Letters, vol. 25, no. 21, pp. 2035-2038, Nov. 2013.

[141] W. Li, W. T. Wang, W. H. Sun, L. X. Wang, J. G. Liu, and N. H. Zhu, "Generation of Flat Optical Frequency Comb Using a Single Polarization Modulator and a Brillouin-Assisted Power Equalizer," IEEE Photonics Journal, vol. 6, no. 2, pp. 1-8, Apr. 2014

[142] J. Li, C. Yu, and Z. Li, "Complementary frequency shifter based on polarization modulator used for generation of a high-quality frequency- 
locked multicarrier," Optics Letters, vol. 39, no. 6, pp. 1513-1516, Mar. 2014. [Online]. Available: http://ol.osa.org/abstract.cfm?URI=ol39-6-1513

[143] V. Ataie, E. Myslivets, B.-P. Kuo, N. Alic, and S. Radic, "Spectrally equalized frequency comb generation in multistage parametric mixer with nonlinear pulse shaping," Journal of Lightwave Technology, vol. 32, no. 4, pp. 840-846, Feb. 2014.

[144] V. Ataie, E. Temprana, L. Liu, E. Myslivets, B. Kuo, N. Alic, and S. Radic, "Ultrahigh Count Coherent WDM Channels Transmission Using Optical Parametric Comb-Based Frequency Synthesizer," Journal of Lightwave Technology, vol. 33, no. 3, pp. 694-699, Feb. 2015.

[145] E. Myslivets, B. P. Kuo, N. Alic, and S. Radic, "Generation of wideband frequency combs by continuous-wave seeding of multistage mixers with synthesized dispersion," Optics Express, vol. 20, no. 3, pp. 3331-3344, Jan. 2012. [Online]. Available: http://www.opticsexpress.org/abstract.cfm?URI=oe-20-3-3331

[146] H. Takara, "Supercontinuum multi-carrier source for WDM systems," in Optical Fiber Communication Conference and Exhibit (OFC), Mar. 2002, pp. 314-315.

[147] V. Ataie, B.-P. Kuo, E. Myslivets, and S. Radic, "Generation of 1500tone, $120 \mathrm{~nm}$-wide ultraflat frequency comb by single CW source," in Optical Fiber Communication Conference and Exposition and the National Fiber Optic Engineers Conference (OFC/NFOEC), Mar. 2013, pp. 1-3.

[148] G. Sefler and K. Kitayama, "Frequency comb generation by fourwave mixing and the role of fiber dispersion," Journal of Lightwave Technology, vol. 16, no. 9, pp. 1596-1605, Sept. 1998.

[149] A. Ishizawa, T. Nishikawa, A. Mizutori, H. Takara, S. Aozasa, A. Mori, H. Nakano, A. Takada, and M. Koga, "Octave-spanning frequency comb generated by 250 fs pulse train emitted from $25 \mathrm{GHz}$ externally phase-modulated laser diode for carrier-envelope-offset-locking," Electronics Letters, vol. 46, no. 19, pp. 1343-1344, Sept. 2010.

[150] V. R. Supradeepa and A. M. Weiner, "Bandwidth scaling and spectral flatness enhancement of optical frequency combs from phase-modulated continuous-wave lasers using cascaded four-wave mixing," Optics Letters, vol. 37, no. 15, pp. 3066-3068, Aug. 2012 [Online]. Available: http://ol.osa.org/abstract.cfm?URI=ol-37-15-3066

[151] Y. Liu, A. J. Metcalf, V. T. Company, R. Wu, L. Fan, L. T. Varghese, M. Qi, and A. M. Weiner, "Bandwidth scaling of a phase-modulated continuous-wave comb through four-wave mixing in a silicon nanowaveguide," Optics Letters, vol. 39, no. 22, pp. 6478-6481, Nov. 2014. [Online]. Available: http://ol.osa.org/abstract.cfm?URI=ol-39-22-6478

[152] Z. Tong, A. O. J. Wiberg, E. Myslivets, B. P. P. Kuo, N. Alic, and S. Radic, "Spectral linewidth preservation in parametric frequency combs seeded by dual pumps," Optics Express, vol. 20, no. 16, pp. 17610-17619, July 2012. [Online]. Available: http://www.opticsexpress.org/abstract.cfm?URI=oe-20-16-17610

[153] R. Wu, V. Torres-Company, D. E. Leaird, and A. M. Weiner, "Supercontinuum-based 10-GHz flat-topped optical frequency comb generation," Optics Express, vol. 21, no. 5, pp. 6045-6052, Mar. 2013.

[154] W. J. Tomlinson, R. H. Stolen, and A. M. Johnson, "Optical wave breaking of pulses in nonlinear optical fibers," Optics Letters, vol. 10, no. 9, pp. 457-459, Sept. 1985. [Online]. Available: http://ol.osa.org/abstract.cfm?URI=ol-10-9-457

[155] A. Gnauck, B. Kuo, E. Myslivets, R. Jopson, M. Dinu, J. Simsarian, P. Winzer, and S. Radic, "Comb-Based 16-QAM Transmitter Spanning the $\mathrm{C}$ and L Bands," IEEE Photonics Technology Letters, vol. 26, no. 8, pp. 821-824, Apr. 2014

[156] E. Temprana, V. Ataie, B.-P. Kuo, E. Myslivets, N. Alic, and S. Radic, "Dynamic reconfiguration of parametric frequency comb for superchannel and flex-grid transmitters," in European Conference on Optical Communication (ECOC), Sept. 2014, pp. 1-3.

[157] T. Yang, J. Dong, S. Liao, D. Huang, and X. Zhang, "Comparison analysis of optical frequency comb generation with nonlinear effects in highly nonlinear fibers," Optics Express, vol. 21, no. 7, pp. 85088520, Apr. 2013.

[158] N. Alic and S. Radic, "Optical frequency combs for telecom and datacom applications," in Optical Fiber Communication Conference. Optical Society of America, Mar. 2014, p. W4E.4. [Online]. Available: http://www.opticsinfobase.org/abstract.cfm?URI=OFC-2014-W4E.4

[159] M. Kourogi, T. Enami, and M. Ohtsu, "A coupled-cavity monolithic optical frequency comb generator," IEEE Photonics Technology Letters, vol. 8, no. 12, pp. 1698-1700, Dec. 1996.

[160] M. Kourogi, K. Nakagawa, and M. Ohtsu, "Wide-span optical frequency comb generator for accurate optical frequency difference measurement," IEEE Journal of Quantum Electronics, vol. 29, no. 10, pp. 2693-2701, Oct. 1993.
[161] T. Kobayashi, T. Sueta, Y. Cho, and Y. Matsuo, "High repetition rate optical pulse generator using a Fabry Perot electro-optic modulator," Applied Physics Letters, vol. 21 , no. 8, pp. 341-343, Oct. 1972. [Online]. Available: http://scitation.aip.org/content/aip/journal/apl/21/8/10.1063/1.1654403

[162] K. Imai, B. Widiyatmoko, M. Kourogi, and M. Ohtsu, " $12-\mathrm{THz}$ frequency difference measurements and noise analysis of an optical frequency comb in optical fibers," IEEE Journal of Quantum Electronics, vol. 35, no. 4, pp. 559-564, Apr. 1999.

[163] Z. Jiang, D. E. Leaird, C. B. Huang, H. Miao, M. Kourogi, K. Imai, and A. M. Weiner, "Spectral line-by-line pulse shaping on an optical frequency comb generator," IEEE Journal of Quantum Electronics, vol. 43, no. 12, pp. 1163-1174, Dec. 2007.

[164] S. Bennett, B. Cai, E. Burr, O. Gough, and A. J. Seeds, "1.8-THz bandwidth, zero-frequency error, tunable optical comb generator for DWDM applications," IEEE Photonics Technology Letters, vol. 11, no. 5, pp. 551-553, May 1999.

[165] J. Li, X. Zhang, and Z. Li, "Optical frequency comb generation by utilizing the three-branch waveguide interferometer-based singlesideband modulator with recirculating frequency shifting loop,' Optical Engineering, vol. 53, no. 12, p. 122606, Aug. 2014. [Online]. Available: http://dx.doi.org/10.1117/1.OE.53.12.122606

[166] K.-P. Ho and J. Kahn, "Optical frequency comb generator using phase modulation in amplified circulating loop," IEEE Photonics Technology Letters, vol. 5, no. 6, pp. 721-725, June 1993.

[167] P. Shen, N. Gomes, P. Davies, P. Huggard, and B. N. Ellison, "Analysis and demonstration of a fast tunable fiber-ring-based optical frequency comb generator," Journal of Lightwave Technology, vol. 25, no. 11, pp. 3257-3264, Nov. 2007.

[168] P. Shen, N. Gomes, P. Davies, and W. Shillue, "Generation of 2 $\mathrm{THz}$ Span Optical Comb in a Tunable Fiber Ring Based Optical Frequency Comb Generator," in IEEE International Topical Meeting on Microwave Photonics, Oct. 2007, pp. 46-49.

[169] J. Zhang, N. Chi, and J. Yu, "Generation of coherent and frequencylock multi-carriers using cascaded phase modulators and recirculating frequency shifter for Tb/s optical communication," Optics Express, vol. 19, no. 14, pp. 12891-12902, July 2011. [Online]. Available: http://www.opticsexpress.org/abstract.cfm?URI=oe-19-14-12891

[170] J. Yu, Z. Dong, X. Xiao, Y. Xia, S. Shi, C. Ge, W. Zhou, N. Chi, and Y. Shao, "Generation, transmission and coherent detection of $11.2 \mathrm{~Tb} / \mathrm{s}$ $(112 \times 100 \mathrm{~Gb} / \mathrm{s})$ single source optical OFDM superchannel," in Optical Fiber Communication Conference and Exposition and the National Fiber Optic Engineers Conference (OFC/NFOEC), Mar. 2011, pp. 1-3.

[171] J. Zhang, J. Yu, Z. Dong, Y. Shao, and N. Chi, "Generation of full C-band coherent and frequency-lock multi-carriers by using recirculating frequency shifter loops based on phase modulator with external injection," Optics Express, vol. 19, no. 27, pp. 26370-26381, Dec. 2011.

[172] N. Dupuis, C. Doerr, L. Zhang, L. Chen, N. Sauer, P. Dong, L. Buhl, and D. Ahn, "InP-Based Comb Generator for Optical OFDM," Journal of Lightwave Technology, vol. 30, no. 4, pp. 466-472, Feb. 2012

[173] A. Lowery, "Performance predictions and topology improvements for optical serrodyne comb generators," Journal of Lightwave Technology, vol. 23, no. 8, pp. 2371-2379, Aug. 2005.

[174] L. M. Johnson and C. H. Cox, "Serrodyne optical frequency translation with high sideband suppression," Journal of Lightwave Technology, vol. 6, no. 1, pp. 109-112, Jan. 1988

[175] I. Tomita, H. Sanjoh, E. Yamada, and Y. Yoshikuni, "Novel method for generating multiple wavelengths by pulsed serrodyne modulation," IEEE Photonics Technology Letters, vol. 15, no. 9, pp. 1204-1206, Sept. 2003.

[176] M. Endo, "Optical frequency comb generator using a single-sideband suppressed-carrier modulation in an amplified circulating fiber loop," Laser Physics, vol. 12(4), pp. 679-683, 2002.

[177] T. KAWANISHI, T. SAKAMOTO, S. SHINADA, and M. IZUTSU, "Optical frequency comb generator using optical fiber loops with single-sideband modulation," IEICE Electronics Express, vol. 1, no. 8, pp. 217-221, Jan. 2004

[178] J. Li, X. Li, X. Zhang, F. Tian, and L. Xi, "Analysis of the stability of recirculating frequency shifter used as a multi-tone wideband light source for $\mathrm{Tb} / \mathrm{s}$ multi-carrier optical transmission," in 36th European Conference and Exhibition on Optical Communication (ECOC), Sept. 2010, pp. 1-3.

[179] J. Li, K. Worms, R. Maestle, D. Hillerkuss, W. Freude, and J. Leuthold, "Free-space optical delay interferometer with tunable delay and phase," Optics Express, vol. 19, no. 12, pp. 11654-11 666, June 2011. 
[180] F. Tian, X. Zhang, J. Li, and L. Xi, "Generation of 50 Stable FrequencyLocked Optical Carriers for Tb/s Multicarrier Optical Transmission Using a Recirculating Frequency Shifter," Journal of Lightwave Technology, vol. 29, no. 8, pp. 1085-1091, Apr. 2011.

[181] F. Simoes, D. Pataca, and M. Rocha, "Design of a Comb Generator for High Capacity Coherent-WDM Systems," IEEE Latin America Transactions (Revista IEEE America Latina), vol. 10, no. 3, pp. 16901696, Apr. 2012.

[182] J. Li, X. Zhang, and L. Xi, "Generation of stable and high-quality frequency-locked carriers based on improved recirculating frequency shifter," Optics Communications, vol. 285 , no. 20, pp. 4072-4075, Sept. 2012. [Online]. Available: http://www.sciencedirect.com/science/article/pii/S0030401812005548

[183] J. Li, L. Xi, X. Tang, J. Lin, X. Zhang, H. Cheng, and $X$. Zhang, "Analysis of performance of RFS-based optical comb influenced by linewidth of laser source," Chinese Optical Letters, vol. 12, no. 2, p. 020601, Feb. 2014. [Online]. Available: http://col.osa.org/abstract.cfm?URI=col-12-2-020601

[184] A. Kanno and T. Kawanishi, "Phase noise analysis of an optical frequency comb using single side-band suppressed carrier modulation in an amplified optical fiber loop," IEICE Electronics Express, vol. 9, no. 18, pp. 1473-1478, 2012.

[185] Y. Yu, C. Lei, M. Chen, H. Chen, S. Yang, S. Wu, and S. Xie, "90tone stable optical frequency comb generation using a recirculating frequency shifter," in Asia Communications and Photonics Conference (ACP), Nov. 2012, pp. 1-3.

[186] J. Zhang, J. Yu, N. Chi, Y. Shao, L. Tao, Y. Wang, and X. Li, "Improved multicarriers generation by using multifrequency shifting recirculating loop," IEEE Photonics Technology Letters, vol. 24, no. 16, pp. 14051408, Aug. 2012.

[187] J. Zhang, J. Yu, N. Chi, Z. Dong, Y. Shao, L. Tao, and X. Li, "Theoretical and experimental study on improved frequency-locked multicarrier generation by using recirculating loop based on multifrequency shifting single-sideband modulation," IEEE Photonics Journal, vol. 4, no. 6, pp. 2249-2261, Dec. 2012.

[188] J. Zhang, J. Yu, N. Chi, Y. Shao, L. Tao, J. Zhu, and Y. Wang, "Stable Optical Frequency-Locked Multicarriers Generation by Double Recirculating Frequency Shifter Loops for Tb/s Communication," Journal of Lightwave Technology, vol. 30, no. 24, pp. 3938-3945, Dec. 2012.

[189] J. Lin, L. Xi, J. Li, X. Zhang, X. Zhang, and S. A. Niazi, "Low noise optical multi-carrier generation using optical-FIR filter for ASE noise suppression in re-circulating frequency shifter loop," Optics Express, vol. 22, no. 7, pp. 7852-7864, Apr. 2014.

[190] X. Zhang, J. Lin, X. Zhang, L. Xi, and W. Zhang, "A novel scheme for noise suppression in optical comb generation," in 6th International Conference on Advanced Infocomm Technology (ICAIT), July 2013, pp. 13-14.

[191] C. Lei, H. Chen, M. Chen, S. Yang, and S. Xie, "Recirculating frequency shifting based wideband optical frequency comb generation by phase coherence control," IEEE Photonics Journal, vol. 7, no. 1, pp. 1-7, Feb. 2015.

[192] X. Li, J. Yu, Z. Dong, J. Zhang, Y. Shao, and N. Chi, "Multi-channel multi-carrier generation using multi-wavelength frequency shifting recirculating loop," Optics Express, vol. 20, no. 20, pp. 21 833-21 839, Sept. 2012.

[193] J. Li and Z. Li, "Frequency-locked multicarrier generator based on a complementary frequency shifter with double recirculating frequencyshifting loops," Optics Letters, vol. 38, no. 3, pp. 359-361, Feb. 2013. [Online]. Available: http://ol.osa.org/abstract.cfm?URI=ol-38-3-359

[194] J. Zhang, J. Yu, Z. Dong, N. Chi, and X. Li, "Multi-channel optical frequency-locked multi-carrier source generation based on multi-channel recirculation frequency shifter loop," in Optical Fiber Communication Conference and Exposition and the National Fiber Optic Engineers Conference (OFC/NFOEC), Mar. 2013, pp. 1-3.

[195] Y. Ma, Q. Yang, Y. Tang, S. Chen, and W. Shieh, "1-Tb/s per channel coherent optical OFDM transmission with subwavelength bandwidth access," in Conference on Optical Fiber Communication - incudes post deadline papers (OFC), Mar. 2009, pp. 1-3.

[196] J. Lin, L. Xi, J. Li, J. Li, X. Tang, L. Sun, and X. Zhang, "High-quality frequency-locked optical frequency comb source for terabits optical communication system," Optical Engineering, vol. 53, no. 12, p. 122608, Sept. 2014. [Online]. Available: http://dx.doi.org/10.1117/1.OE.53.12.122608

[197] Thorlabs, "www.thorlabs.com/catalogpages/693.pdf," Tech. Rep.

[198] https://www.rp-photonics.com/periodicpoling.html, Std.

[199] E. G. Sauter, Nonlinear optics. Wiley Series in Microwave and Optical Engineering, New York, USA. Wiley and Sons, 1996.
[200] C. R. Phillips, C. Langrock, J. S. Pelc, M. M. Fejer, J. Jiang, M. E. Fermann, and I. Hartl, "Supercontinuum generation in quasiphase-matched $\mathrm{LiNbO} 3$ waveguide pumped by a Tm-doped fiber laser system," Optics Letters, vol. 36, no. 19, pp. 3912-3914, Oct. 2011. [Online]. Available: http://ol.osa.org/abstract.cfm?URI=ol-36-19-3912

[201] M. Scaffardi, S. Pinna, E. Lazzeri, and A. Bogoni, "Generation of a flexible optical comb in a periodically poled lithium niobate waveguide," Optics Letters, vol. 39, no. 7, pp. 1733-1736, Apr. 2014. [Online]. Available: http://ol.osa.org/abstract.cfm?URI=ol-39-7-1733

[202] V. Vercesi, S. Pinna, G. Meloni, F. Scotti, L. Potì, A. Bogoni, and M. Scaffardi, "Flexible frequency comb generation in a periodically poled lithium niobate waveguide enabling optical multicasting," Optics Letters, vol. 39, no. 20, pp. 5981-5984, Oct. 2014. [Online]. Available: http://ol.osa.org/abstract.cfm?URI=ol-39-20-5981

[203] M. Scaffardi, V. Vercesi, A. Sgambelluri, and A. Bogoni, "Hitless Reconfiguration of a PPLN-Based Multiwavelength Source for Elastic Optical Networks," Journal of Optical Communication and Networking, vol. 8, no. 2, pp. 85-92, Feb. 2016. [Online]. Available: http://jocn.osa.org/abstract.cfm?URI=jocn-8-2-85

[204] J. van der Ziel and R. Logan, "Generation of short optical pulses in semiconductor lasers by combined DC and microwave current injection," IEEE Journal of Quantum Electronics, vol. 18, no. 9, pp. 1340-1350, Sept. 1982.

[205] P. Paulus, R. Langenhorst, and D. Jager, "Generation and optimum control of picosecond optical pulses from gain-switched semiconductor lasers," IEEE Journal of Quantum Electronics, vol. 24, no. 8, pp. 15191523, Aug. 1988.

[206] S. Tarucha and K. Otsuka, "Response of semiconductor laser to deep sinusoidal injection current modulation," IEEE Journal of Quantum Electronics, vol. 17, no. 5, pp. 810-816, May 1981.

[207] A. G. Weber, W. Ronghan, E. H. Bottcher, M. Schell, and D. Bimberg, "Measurement and simulation of the turn-on delay time jitter in gainswitched semiconductor lasers," IEEE Journal of Quantum Electronics, vol. 28, no. 2, pp. 441-446, Feb. 1992.

[208] M. Schell, D. Huhse, W. Utz, J. Kaessner, D. Bimberg, and I. S. Tarasov, "Jitter and dynamics of self-seeded Fabry-Perot laser diodes," IEEE Journal of Selected Topics in Quantum Electronics, vol. 1, no. 2, pp. 528-534, June 1995.

[209] S. Duill, R. Zhou, P. Anandarajah, and L. Barry, "Numerical investigation into the dynamics of externally-injected, gain-switched lasers for optical comb generation," in European Conference on Optical Communication (ECOC), Sept. 2014, pp. 1-3.

[210] P. M. Anandarajah, L. P. Barry, A. Kaszubowska, J. O'Gorman, J. O'Carroll, C. Herbert, R. Phelan, and A. S. Duke, "Highly coherent picosecond pulse generation with sub-ps jitter and high SMSR by gain switching Discrete Mode laser diodes at $10 \mathrm{GHz}$ line rate," in Conference on Optical Fiber Communication - incudes post deadline papers, Mar. 2009, pp. 1-3.

[211] R. Maher, K. Shi, L. P. Barry, J. O'Carroll, B. Kelly, R. Phelan, J. O'Gorman, and P. M. Anandarajah, "Implementation of a costeffective optical comb source in a WDM-PON with $10.7 \mathrm{~Gb} / \mathrm{s}$ data to each ONU and 50km reach," Optics express, vol. 18, no. 15, pp. $15672-15681$, July 2010.

[212] R. Maher, P. Anandarajah, S. Ibrahim, L. Barry, A. D. Ellis, P. Perry, R. Phelan, B. Kelly, and J. O'Gorman, "Low cost comb source in a coherent wavelength division multiplexed system," in 36th European Conference and Exhibition on Optical Communication (ECOC), Sept. 2010, pp. 1-3.

[213] P. Anandarajah, R. Maher, Y. Xu, S. Latkowski, J. O'Carroll, S. Murdoch, R. Phelan, J. O'Gorman, and L. Barry, "Generation of coherent multicarrier signals by gain switching of discrete mode lasers," IEEE Photonics Journal, vol. 3, no. 1, pp. 112-122, Feb. 2011.

[214] S. Latkowski, Y. Q. Xu, S. G. Murdoch, L. P. Barry, and P. M. Anandarajah, "Optical comb generation and expansion by gain switched discrete mode laser diode," in Conference on Lasers and Electro-Optics Europe and 12th European Quantum Electronics Conference (CLEO EUROPE/EQEC), May 2011, pp. 1-1.

[215] A. Criado Serrano, C. de Dios Fernandez, E. Prior Cano, M. Ortsiefer, P. Meissner, and P. Acedo, "VCSEL-Based Optical Frequency Combs: Toward Efficient Single-Device Comb Generation," IEEE Photonics Technology Letters, vol. 25, no. 20, pp. 1981-1984, Oct. 2013.

[216] A. P. Anthur, R. T. Watts, R. Zhou, P. Anandarajah, D. Venkitesh, and L. P. Barry, "Penalty-free wavelength conversion with variable channel separation using gain-switched comb source," Optics Communications, vol. 324, pp. 69-72, Aug. 2014.

[217] P. M. Anandarajah, S. P. Duill, R. Zhou, and L. P. Barry, "Enhanced optical comb generation by gain-switching a single-mode semiconduc- 
tor laser close to its relaxation oscillation frequency," IEEE Journal of Selected Topics in Quantum Electronics, vol. 21, no. 6, pp. 592-600, Nov. 2015.

[218] P. M. Anandarajah, T. N. Huynh, V. Vujicic, R. Zhou, and L. P. Barry, "UDWDM PON with 6 X 2.5 GBaud 16-QAM Multicarrier Transmitter and Phase Noise Tolerant Direct Detection," in Optical Fiber Communication Conference. Optical Society of America, Mar. 2015, pp. Th2A-58.

[219] R. Zhou, S. Latkowski, J. O'Carroll, R. Phelan, L. Barry, and P. Anandarajah, "40 nm wavelength tunable gain-switched optical comb source," in 37th European Conference and Exhibition on Optical Communication (ECOC), Sept. 2011, pp. 1-3.

[220] M. D. G. Pascual, R. Zhou, F. Smyth, P. M. Anandarajah, and L. P. Barry, "Software reconfigurable highly flexible gain switched optical frequency comb source," Optics Express, vol. 23, no. 18, pp. 23 225-23235, Sept. 2015. [Online]. Available: http://www.opticsexpress.org/abstract.cfm?URI=oe-23-18-23225

[221] P. Anandarajah, R. Zhou, R. Maher, M. Gutierrez Pascual, F. Smyth, V. Vujicic, and L. Barry, "Flexible optical comb source for super channel systems," in Optical Fiber Communication Conference and Exposition and the National Fiber Optic Engineers Conference (OFC/NFOEC), Mar. 2013, pp. 1-3.

[222] P. M. Anandarajah, R. Zhou, R. Maher, D. Lavery, M. Paskov, B. Thomsen, S. Savory, and L. P. Barry, "Gain-switched multicarrier transmitter in a long-reach UDWDM PON with a digital coherent receiver," Optics Letters, vol. 38, no. 22, pp. 4797-4800, Nov. 2013.

[223] R. Zhou, P. M. Anandarajah, R. Maher, M. Paskov, D. Lavery, B. C. Thomsen, S. J. Savory, and L. P. Barry, "80-km coherent DWDMPON on 20-GHz grid with injected gain switched comb source," IEEE Photonics Technology Letters, vol. 26, no. 4, pp. 364-367, 2014.

[224] P. M. Anandarajah, R. Zhou, V. Vujicic, D. G. Pascual, E. Martin, and L. P. Barry, "Long reach UDWDM PON with SCM-QPSK modulation and direct detection," in Optical Fiber Communication Conference. Optical Society of America, 2014, pp. W2A-42.

[225] J. Pfeifle, V. Vujicic, R. T. Watts, P. C. Schindler, C. Weimann, R. Zhou, W. Freude, L. P. Barry, and C. Koos, "Flexible terabit/s NyquistWDM super-channels using a gain-switched comb source," Optics Express, vol. 23, no. 2, pp. 724-738, Jan. 2015. [Online]. Available: http://www.opticsexpress.org/abstract.cfm?URI=oe-23-2-724

[226] R. Zhou, P. M. Anandarajah, D. G. Pascual, J. O'Carroll, R. Phelan, B. Kelly, and L. P. Barry, "Monolithically integrated 2-section lasers for injection locked gain switched comb generation," in Optical Fiber Communication Conference. Optical Society of America, Mar. 2014, p. Th3A.3. [Online]. Available: http://www.opticsinfobase.org/abstract.cfm?URI=OFC-2014-Th3A.3

[227] P. M. Anandarajah, T. Shao, R. Zhou, D. G. Pascual, and L. P. Barry, "100Gb/s WDM-SSB-DD-OFDM using a Gain Switched Monolithically Integrated Passive Feedback Comb Source," in CLEO: Science and Innovations. Optical Society of America, May 2015, pp. SF2K-1.

[228] M. Deseada Gutierrez Pascual, P. Anandarajah, R. Zhou, F. Smyth, S. Latkowski, and L. Barry, "Cascaded Fabry-Perot Lasers for Coherent Expansion of Wavelength Tunable Gain Switched Comb," in European Conference on Optical Communication (ECOC), Sept. 2014, pp. 1-3.

[229] M. D. G. Pascual, R. Zhou, F. Smyth, T. Shao, P. M. Anandarajah, and L. Barry, "Dual mode injection locking of a Fabry-Perot laser for tunable broadband gain switched comb generation," in European Conference on Optical Communication (ECOC), Sept. 2015, pp. 1-3.

[230] F. A. Gutiérrez, E. P. Martin, P. Perry, A. D. Ellis, P. Anandarajah, F. Smyth, and L. P. Barry, "100 Gbit/s real-time all-analogue filter bank OFDM based on a gain-switched optical comb," in European Conference on Optical Communication (ECOC), Sept. 2015, pp. 1-3.

[231] M. D. Gutierrez, J. Braddell, F. Smyth, and L. P. Barry, "Monolithically integrated lasers for comb generation in bandwidth variable transponders," in 18th European Conference on Integrated Optics (ECIO), May 2016. [Online]. Available: http://www.ecioconference.org/wp-content/uploads/2016/06/ECIO-o-01.pdf

[232] F. A. Gutierrez, E. P. Martin, P. Perry, A. D. Ellis, P. M. Anandarajah, and L. P. Barry, "WDM Orthogonal Subcarrier Multiplexing," Journal of Lightwave Technology, vol. 34, no. 8, pp. 1815-1823, Apr. 2016.

[233] M. D. G. Pascual, V. Vujicic, J. Braddell, F. Smyth, P. M. Anandarajah, and L. P. Barry, "InP photonic integrated externally injected gain switched optical frequency comb," Optics Letters, vol. 42, no. 3, pp. 555-558, Feb. 2017. [Online]. Available: http://ol.osa.org/abstract.cfm?URI=ol-42-3-555

[234] M. D. G. Pascual, V. Vujicic, J. Braddell, F. Smyth, P. Anandarajah, and L. Barry, "Photonic integrated gain switched optical frequency comb for spectrally efficient optical transmission systems," IEEE Photonics Journal, vol. 9, no. 3, pp. 1-8, June 2017.

[235] P. Del'Haye, A. Schliesser, O. Arcizet, T. Wilken, R. Holzwarth, and T. J. Kippenberg, "Optical frequency comb generation from a monolithic microresonator," Nature, vol. 450, pp. 1214-1217, Dec. 2007.

[236] P. Del'Haye, B. Ketja, S. B. Papp, and S. A. Diddams, "SelfInjection Locking and Phase-Locked States in MicroresonatorBased Optical Frequency Combs," Physical Review Letters, vol. 112, p. 043905, Jan. 2014. [Online]. Available: http://link.aps.org/doi/10.1103/PhysRevLett.112.043905

[237] I. S. Grudinin, N. Yu, and L. Maleki, "Generation of optical frequency combs with a $\mathrm{CaF} 2$ resonator," Optics Letters, vol. 34, no. 7, pp. 878-880, Apr. 2009. [Online]. Available: http://ol.osa.org/abstract.cfm?URI=ol-34-7-878

[238] A. A. Savchenkov, A. B. Matsko, V. S. Ilchenko, I. Solomatine, D. Seidel, and L. Maleki, "Tunable optical frequency comb with a crystalline whispering gallery mode resonator," Physical Review Letters, vol. 101, p. 093902, Aug. 2008. [Online]. Available: http://link.aps.org/doi/10.1103/PhysRevLett.101.093902

[239] C. Y. Wang, T. Herr, P. Del'Haye, A. Schliesser, J. Hofer, R. Holzwarth T. W. Hänsch, N. Picqué, and T. J. Kippenberg, "Mid-infrared optical frequency combs based on crystalline microresonators," arXiv:1109.2716, Sept. 2011.

[240] W. Liang, A. A. Savchenkov, A. B. Matsko, V. S. Ilchenko, D. Seidel, and L. Maleki, "Generation of near-infrared frequency combs from a MgF2 whispering gallery mode resonator," Optics Letters, vol. 36, no. 12, pp. 2290-2292, June 2011. [Online]. Available: http://ol.osa.org/abstract.cfm?URI=ol-36-12-2290

[241] I. H. Agha, Y. Okawachi, and A. L. Gaeta, "Theoretical and experimental investigation of broadband cascaded fourwave mixing in high-Q microspheres," Optics Express, vol. 17, no. 18, pp. 16209-16215, Aug. 2009. [Online]. Available: http://www.opticsexpress.org/abstract.cfm?URI=oe-17-18-16209

[242] L. Razzari, D. Duchesne, M. Ferrera, R. Morandotti, S. Chu, B. E. Little, and D. J. Moss, "CMOS-compatible integrated optical hyperparametric oscillator," Nature Photonics, vol. 4, p. 41, Jan. 2010.

[243] J. S. Levy, A. Gondarenko, M. A. Foster, A. C. Turner-Foster, A. L. Gaeta, and M. Lipson, "CMOS-compatible multiple-wavelength oscillator for on-chip optical interconnects," Nature Photonics, vol. 4, pp. 37-40, Jan. 2010.

[244] M. A. Foster, J. S. Levy, O. Kuzucu, K. Saha, M. Lipson, and A. L. Gaeta, "Silicon-based monolithic optical frequency comb source," Optics Express, vol. 19, no. 15, pp. 14 233-14 239, July 2011. [Online] Available: http://www.opticsexpress.org/abstract.cfm?URI=oe-19-1514233

[245] Y. Okawachi, K. Saha, J. S. Levy, M. A. Foster, M. Lipson, and A. L. Gaeta, "Ultrabroadband frequency comb generation at 1micrometer in a silicon-nitride ring resonator," in CLEO: Laser Applications to Photonic Applications. Optical Society of America, May 2011, p. CFK2.

[246] Y. Okawachi, K. Saha, J. S. Levy, Y. H. Wen, M. Lipson, and A. L. Gaeta, "Octave-spanning frequency comb generation in a silicon nitride chip," Optics Letters, vol. 36, no. 17, pp. 3398-3400, Sept. 2011. [Online]. Available: http://ol.osa.org/abstract.cfm?URI=ol-36-17-3398

[247] F. Ferdous, H. Miao, D. E. Leaird, K. Srinivasan, J. Wang, L. Chen, L. T. Varghese, and A. M. Weiner, "Spectral line-by-line pulse shaping of on-chip microresonator frequency combs," Nature Photonics, vol. 5, p. 770 , Dec. 2011

[248] T. J. Kippenberg, R. Holzwarth, and S. A. Diddams, "Microresonator-based optical frequency combs," Science, vol. 332, no. 6029, pp. 555-559, Apr. 2011. [Online]. Available: http://www.sciencemag.org/content/332/6029/555.abstract

[249] P. Del'Haye, "Optical frequency comb generation in monolithic microresonators," in https://core.ac.uk/display/11032610, 2011.

[250] P. Del'Haye, O. Arcizet, A. Schliesser, R. Holzwarth, and T. J. Kippenberg, "Full stabilization of a microresonatorbased optical frequency comb," Physical Review Letters, vol. 101, p. 053903, July 2008. [Online]. Available: http://link.aps.org/doi/10.1103/PhysRevLett.101.053903

[251] J. S. Levy, K. Saha, Y. Okawachi, M. A. Foster, A. L. Gaeta, and M. Lipson, "High-performance silicon-nitride-based multiplewavelength source," IEEE Photonics Technology Letters, vol. 24, no. 16, pp. 1375-1377, Aug. 2012.

[252] J. Pfeifle, V. Brasch, M. Lauermann, Y. Yu, D. Wegner, T. Herr, K. Hartinger, P. Schindler, J. Li, D. Hillerkuss, R. Schmogrow, C. Weimann, R. Holzwarth, W. Freude, J. Leuthold, T. J. Kippenberg, and C. Koos, "Coherent terabit communications 
with microresonator Kerr frequency combs," Nature Photoncs, vol. 8, no. 5, pp. 375-380, May 2014. [Online]. Available: http://dx.doi.org/10.1038/nphoton.2014.57

[253] J. Pfeifle, A. Kordts, P. Marin, M. Karpov, M. Pfeiffer, V. Brasch, R. Rosenberger, J. Kemal, S. Wolf, W. Freude, T. J. Kippenberg, and C. Koos, "Full C and L-band transmission at $20 \mathrm{Tbit} / \mathrm{s}$ using cavity-soliton Kerr frequency combs," in Conference on Lasers and Electro-Optics (CLEO), May 2015, pp. 1-2.

[254] J. Yang, S. W. Huang, H. Zhou, M. Yu, D. L. Kwong, and C. W. Wong, "A low-phase-noise $18 \mathrm{GHz}$ Kerr frequency comb spanning $65 \mathrm{THz}$," in Optical Fiber Communications Conference and Exhibition (OFC), Mar. 2015, pp. 1-3.

[255] Y. Xuan, Y. Liu, X. Xue, P. H. Wang, J. Wang, B. Niu, K. Han, M. Teng, D. E. Leaird, A. M. Weiner, and M. Qi, "High-Q silicon nitride microresonator for low power frequency comb initiation at normal dispersion regime," in Conference on Lasers and Electro-Optics (CLEO), May 2015, pp. 1-2.

[256] B. J. Puttnam, R. S. Luís, W. Klaus, J. Sakaguchi, J. M. D. Mendinueta, Y. Awaji, N. Wada, Y. Tamura, T. Hayashi, M. Hirano, and J. Marciante, "2.15 Pb/s transmission using a 22 core homogeneous single-mode multi-core fiber and wideband optical comb," in European Conference on Optical Communication (ECOC), Sept. 2015, pp. 1-3.

[257] X. Sun, L. Zhou, J. Xie, Z. Zou, L. Lu, H. Zhu, X. Li, and J. Chen, "Tunable silicon Fabry-Perot comb filters formed by Sagnac loop mirrors," Optics Letters, vol. 38, no. 4, pp. 567-569, Feb. 2013. [Online]. Available: http://ol.osa.org/abstract.cfm?URI=ol-38-4-567

[258] C. Zhong, C. Shen, K. Li, X. Dong, and Y. Jin, "Spectral Characteristics of Multiple and Cascaded Phase-Shifted Fiber Bragg Grating," in Symposium on Photonics and Optoelectronics (SOPO), May 2011, pp. $1-4$.

[259] S. Kamei, Y. Inoue, T. Shibata, and A. Kaneko, "Low-loss and compact silica-based athermal arrayed waveguide grating using resinfilled groove," Journal of Lightwave Technology, vol. 27, no. 17, pp. 3790-3799, Sept. 2009.

[260] D. J. F. Barros, J. M. Kahn, J. P. Wilde, and T. A. Zeid, "Bandwidth-scalable long-haul transmission using synchronized colorless transceivers and efficient wavelength-selective switches," Journal of Lightwave Technology, vol. 30, no. 16, pp. 2646-2660, Aug. 2012.

[261] M. Iwama, M. Takahashi, Y. Uchida, M. Kimura, R. Kawahara, S. ichi Matsushita, and T. Mukaihara, "Low loss 1 x 93 wavelength selective switch using PLC-based spot size converter," in European Conference on Optical Communication (ECOC). Institute of Electrical and Electronics Engineers (IEEE), Sept. 2015, pp. 1-3.

[262] J. Minowa and Y. Fujii, "Dielectric multilayer thin-film filters for WDM transmission systems," Journal of Lightwave Technology, vol. 1, no. 1, pp. 116-121, Mar. 1983.

[263] T. N. Nguyen, M. Gay, K. Lengla, L. Bramerie, M. Thual, J. C. Simon, S. Malaguti, G. Bellanca, S. Trillo, S. Combria, G. Lehoucq, and A. D. Rossi, " $100-\mathrm{Gb} / \mathrm{s}$ wavelength division demultiplexing using a photonic crystal four-channel drop filter," IEEE Photonics Technology Letters, vol. 25, no. 9, pp. 813-816, May 2013.

[264] F. Lu, G. Li, K. Li, Z. Wang, and A. Xu, "A compact wavelength demultiplexing structure based on arrayed MIM plasmonic nano-disk cavities," Optics Communications, vol. 285, no. 24, pp. 5519-5523, Nov. 2012

[265] H. Kawaguchi, K. Magari, K. Oe, Y. Noguchi, Y. Nakano, and G. Motosugi, "Optical frequency-selective amplification in a distributed feedback type semiconductor laser amplifier," Applied Physics Letters, vol. 50, no. 2, pp. 66-67, Jan. 1987.

[266] F.-S. Choa and T. Koch, "Static and dynamical characteristics of narrow-band tunable resonant amplifiers as active filters and receivers," Journal of Lightwave Technology, vol. 9, no. 1, pp. 73-83, Jan. 1991.

[267] W. Cotter, D. Goulding, B. Roycroft, J. O'Callaghan, B. Corbett, and F. H. Peters, "Investigation of active filter using injection-locked slotted Fabry-Perot semiconductor laser," Applied Optics, vol. 51, no. 30, pp. 7357-7361, Oct. 2012.

[268] R. Zhou, T. Shao, M. D. G. Pascual, F. Smyth, and L. P. Barry, "Injection Locked Wavelength De-Multiplexer for Optical Comb-Based Nyquist WDM System,” IEEE Photonics Technology Letters, vol. 27, no. 24, pp. 2595-2598, Dec. 2015.

[269] R. Zhou, M. D. G. Pascual, P. M. Anandarajah, T. Shao, F. Smyth, and L. P. Barry, "Flexible wavelength de-multiplexer for elastic optical networking," Optics Letters, vol. 41, no. 10, pp. 2241-2244, May 2016.

[270] M. Dallaglio, A. Giorgetti, N. Sambo, and P. Castoldi, "Impact of SBVTs based on multi-wavelength source during provisioning and restoration in elastic optical networks," in The European Conference on Optical Communication (ECOC), Sept. 2014, pp. 1-3.
[271] M. Dallaglio, A. Giorgetti, N. Sambo, L. Velasco, and P. Castoldi, "Impact of multi-wavelength sliceable transponders in elastic optical networks," in Optical Fiber Communications Conference and Exhibition (OFC), Mar. 2015, pp. 1-3.

[272] H. Takara, T. Ohara, K. Mori, K. Sato, E. Yamada, Y. Inoue, T. Shibata, M. Abe, T. Morioka, and K. I. Sato, "More than 1000 channel optical frequency chain generation from single supercontinuum source with $12.5 \mathrm{GHz}$ channel spacing," Electronics Letters, vol. 36, no. 25, pp. 2089-2090, Dec. 2000.

[273] Y. Cai, Y. Yan, J. Cheng, and L. Zhou, "Design and evaluation of elastic optical network with centralized multi-carrier light source," in 22nd Wireless and Optical Communication Conference, May 2013, pp. $506-511$.

[274] Y. Cai, E. Oki, M. Matsuura, N. Kishi, and T. Miki, "Design and evaluation of an optical broadcast-and-select network architecture with a centralized multicarrier light source," Journal of Lightwave Technology, vol. 27, no. 21, pp. 4897-4906, Nov. 2009.

[275] Y. Cai, M. Matsuura, E. Oki, N. Kishi, and T. Miki, "Optical drop-adddrop network architecture with centralized multi-carrier light source," IEEE Communications Letters, vol. 13, no. 9, pp. 700-702, Sept. 2009.

[276] M. Imran, A. D. Errico, A. Lord, and L. Poti, "Techno-economic analysis of carrier sources in slice-able bandwidth variable transponders," in 42nd European Conference on Optical Communication (ECOC), Sept. 2016, pp. 1-3. 


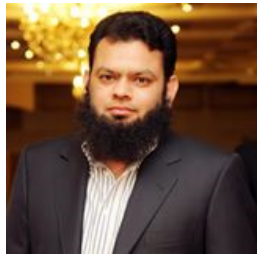

Dr. Muhammad Imran received the B.Sc. Electrical Engineering in 2003 from University of Engineering \& Technology Taxila, Pakistan. From 2004 to 2010, he served in Pakistan Telecommunication Company Limited (PTCL) as Senior Engineer in Optical Fiber Networks Department. During his stay at PTCL, he was involved in many core and metro SDH/DWDM network deployments and has hands on experience on multiple vendors' commercial products in real networks. In 2010, he was selected for Erasmus Mundus Scholarship for Masters on Photonic Network Engineering (MAPNET) and received his double degree from Aston University UK and Scoula Superiore Sant'Anna Italy. From 2012 he is associated with High capacity optical communications systems (HCOC) research group Scoula Superiore Sant'Anna Pisa, Italy where he completed his $\mathrm{PhD}$ and is currently working as post-doctoral researcher. He also worked in British Telecom research labs, UK as visiting research student. His research interests include High capacity optical communications, optical frequency combs and SDM networks.

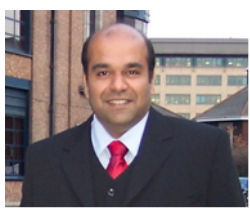

Dr. Prince Anandarajah received the B.Eng (Electronic Engineering) degree from University of Nigeria, Nsukka in 1992. Subsequently, he worked as an Instructor/Maintenance Engineer at the Nigerian College of Aviation Technology. On completing his M.Eng (1998), he joined the Radio and Optical Communications Group at DCU where he obtained his PhD degree (2003). He then worked as a postdoctoral researcher until 2006 and later as a Research Officer with the High Speed Devices and Systems centre which is part of the Rince institute (2007). He then held a DCU senior research fellow position until 2016 and is currently a lecturer with the School of Electronic Engineering at DCU. His main research interests include direct modulation techniques, generation and optimization of optical frequency combs and short optical pulses, spectrally efficient modulation formats and radio-over-fibre distribution systems. He has published over 190 articles in internationally peer reviewed journals and conferences and is also a holder of 5 international patents. He is also a founder and a director of a spin-off company called Pilot Photonics. He is a senior member of the IEEE.

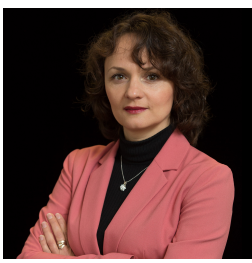

Dr. Aleksandra Kaszubowska-Anandarajah was awarded a PhD from Dublin City University (DCU) in 2004 for work in the area of broadband access networks using hybrid radio/fiber systems. Subsequently, she worked as a postdoctoral researcher at DCU, focusing on Radio over Fibre networks as well as the application of fast tunable lasers in an optical packet switched systems. In 2008, Dr. Kaszubowska joined an Irish start-up company Intune Networks Ltd., as a senior optical Engineer, where she worked on developing world's first optical packet switched network. She returned to academia in 2014, when she joined the CTVR/CONNECT group at Trinity College Dublin, as a strategic research coordinator. Currently, she is working as a principal investigator on a Science Foundation Ireland funded grant. Her main research interests include spectrally efficient modulation formats, flexible optical networks, real-time programmable transmitters and $\mathrm{THz}$ transmission systems. She has published over 70 articles in internationally peer reviewed journals and conferences. She is a reviewer for journals such as IEEE Photonics Technology Letters, IEEE Journal of Lightwave Technology, IEEE Journal of Quantum Electronics and IET Communications. She has also been involved in reviewing several of the European Union framework 7 STREP and Horizon 2020 projects. Dr. Kaszubowska is a member of the IEEE.

Dr. Nicola Sambo is an assistant professor at Scuola Superiore Sant'Anna He was a visiting student at France Telecom, Lannion. He is also collaborating with CNIT, Pisa, Italy. His research interests include optical network architecture, transmission performance modeling, and control plane. He is an author of about 100 publications including international journals, conference proceedings, and patents.

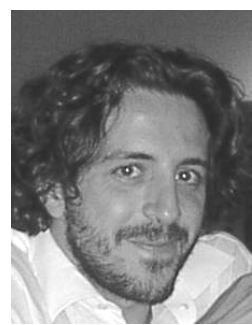

Dr. Luca Potí (M98) was born in Parma, Italy in 1971. He received the M.S. Degree in electronics engineering from University of Parma, Parma, Italy in 1997. From 2012 he is Head of Research Area with the Interuniversity National Consortium for Telecommunications (CNIT) at the Photonic Networks Nat'l Lab and external collaborator for Scuola Superiore Sant'Anna, at the Institute of Communication, Information, and Perception Technologies (TeCIP) both located in Pisa, Italy. In 1997 he was visiting researcher at the Centre d'Optique, Photonique et Laser (COPL), Quebéc, Canada working on recirculating loop experiment for ultra-long haul transmission. In 1998 he received a Marconi Communication grant on the topic: "WDM communication systems on optical fibre" at the Marconi Laboratory of Parma University working on Nonlinear effects due to fibers and EDFAs in WDM systems. From 1999 to 2000 he worked as a responsible for the optical communications laboratory at the University of Parma, Italy. From 2001-2003 he was senior researcher with CNIT at the Photonics Networks National Laboratory in Pisa where he was designed as Head of Research until 2010. In 2002 he was visiting researcher at the National Institute of Information and Communications Technology (NICT), Tokyo, Japan, working on ultra-fast phase comparison for $160 \mathrm{GHz}$ signals. In 2005 he started, together with two researchers, PhoTrix S.r.l. an Italian company producing pulsed fibre lasers and ultra-fast subsystems. $\mathrm{He}$ has published more than 300 international journal papers, conference papers, and patents. He was Scientific Coordinator for the EU Project 'Large Optical Bandwidth by amplifier Systems based on Tellurite fibres doped with Rare earths' (LOBSTER). He served as Coordinator several projects supported by the Italian Ministry of University and Research (MIUR) and Ministry of Foreign Affairs (MAE). His research interests were mainly focused on ultra-fast communication systems, in fact, in 2001 his group demonstrated first Italian transmission system working at $160 \mathrm{Gbit} / \mathrm{s}$. For such purpose strong expertise has been developed in ultra-short optical pulse generation for telecommunication. In 2011 his group demonstrated together with Ericsson the first coherent system working at $448 \mathrm{Gbit} / \mathrm{s}$ on an installed commercial apparatus in field trial hosting real traffic. The same in 2012 system has been upgraded to 1Tbit/s super-channel in a flexible optical network. In 2012 he was invested with the career award PWI (Italian Web Award)for the outstanding research results in the previous 10 years. Recently he is interested in optical coherent systems, wavelength switched optical networks, and optical systems for biomedical application and tribology. 